U.S. Department

of Transportatlon

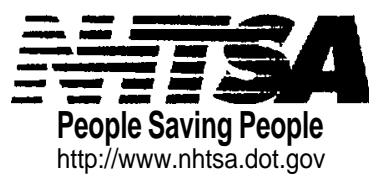

National Highway

Traflic Safety

http://www.nhtsa.dot.gov

\title{
Heavy Vehicle Driver Workload Assessment Task 1: Task Analysis Data and Protocols Review
}

This document is available to the public from the National Technical Information Service, Springfield; Virginia 22161.

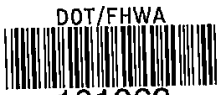


This research was supported (in part) by the National Highway Trqfic Safety Administration (NHTSAA), U.S. Department of Transportation, Under Contract No. DTFNH22-91-C-07003. The opinions, findings and recommendations contained herein are those of the authors, and do not necessarily represent those of the NHTSA. 
Technical Report Documentation Page

\begin{tabular}{|c|c|c|}
\hline 1. Report No. & $\begin{array}{l}\text { 2. Government Accession No. } \\
\text { D O T HS } 808 \quad 467 \text { (1) }\end{array}$ & 3. Recipient's Catalog No. \\
\hline \multirow{2}{*}{\multicolumn{2}{|c|}{$\begin{array}{l}\text { 4. Title and Subtitle } \\
\text { Final Report Supplement -- Task 1: Task Analysis Data and protocols Review }\end{array}$}} & $\begin{array}{l}\text { 5. Report Date } \\
\text { January, } 1992 \\
\end{array}$ \\
\hline & & 6. Performing Organization Code \\
\hline $\begin{array}{l}\text { 7. Author(s) L. Tijerina, S, Kiger, W. Wie } \\
\text { A. Bittner, Jr., T. Nygren, L. Myers, C }\end{array}$ & $\begin{array}{l}\text { rwille, T. Rockwell, B. Kantowitz, } \\
\text { Tolbert, and M. McCallum }\end{array}$ & Performing Organization Report No. \\
\hline 9 Performina Oraanization Name and Address & & 10. Work Unit No. (TRAIS) \\
\hline \multicolumn{2}{|l|}{$\begin{array}{l}\text { Battelle Memorial Institute } \\
505 \text { King Avenue } \\
\text { Columbus, OH 43201-2693 }\end{array}$} & $\begin{array}{l}\text { 11. Contract or Grant No. } \\
\text { DTNH22-91-C-07003 }\end{array}$ \\
\hline & & 13. Type of Report and Period Covered \\
\hline \multirow[t]{2}{*}{ 12. Sponsoring Agency Name and Address } & \multirow{2}{*}{$\begin{array}{l}\text { Office of Crash Avoidance Research } \\
\text { National Highway Traffic Safety } \\
\text { Administration } \\
400 \text { 7th street, NRD-50 } \\
\text { Washington, DC } 20590\end{array}$} & $\begin{array}{l}\text { Final Report } \\
\text { July, } 1991 \text {-September, } 1995\end{array}$ \\
\hline & & 14. Sponsoring Agency Code \\
\hline
\end{tabular}

15. Supplementary Notes

Contracting Officer's Technical Representative: Dr. Michael J. Goodman (NRD-50)

\section{Abstract}

This report contains a review of available task analytic data and protocols pertinent to heavy vehicle operation and determination of the availability and relevance of such data to heavy vehicle driver workload assessment. Additionally, a preliminary consideration of development of safety-relevant criteria was pursued, and relevance of a review of the relationship between risk-taking behavior and workload was examined. Task analysis data included American, Canadian, and European sources which varied substantially in format and content, and were largely oriented to support training and certification. A variety of protocols including activity analysis, interviews and commentary, driving or protocol analysis, critical incident technique, subjective workload ratings, visual allocation measures, on-the-road driver-vehicle peformance monitoring, safety criticality ratings, and rankings were identified. No fully devloped methodologies or criteria were found by which to predict accident rates based on workload level. but actuarial, visual allocation of resources, and lanekeeping approaches were presented.

19. Security Classif. (of this report)
20. Security Classif. (of this Page)
21. No. of Pages 152
22. Price 


\section{TABLE OF CONTENTS}

Page

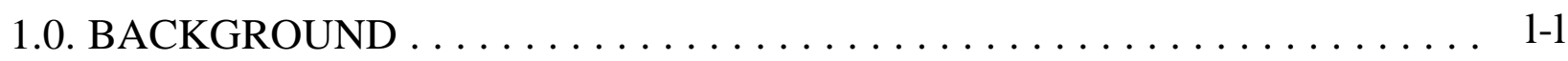

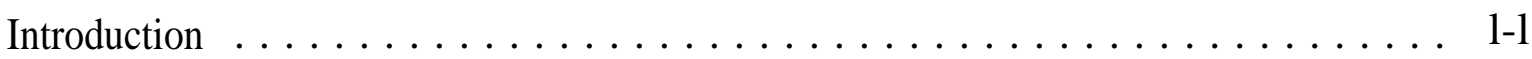

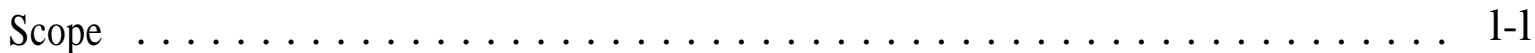

Literature Search . . . . . . . . . . . . . . . . . . . . . . . 1-2

Criteria for Review . . . . . . . . . . . . . . . . . . . . . . . . . $1-2$

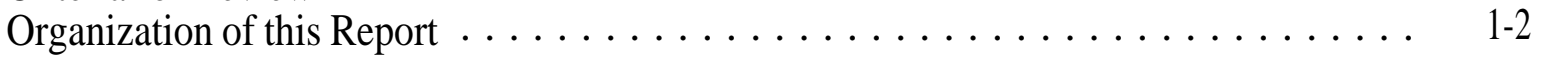

2.0. DRIVING TASKS AND TASK CRITICALITY $\ldots \ldots \ldots \ldots \ldots \ldots \ldots \ldots .2-1$

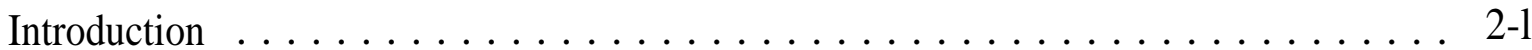

Summary on Task Descriptions and Criticality Assessment $\ldots \ldots \ldots \ldots \ldots \ldots \ldots$ 2-32

APPENDIX 2A. PROFESSIONAL TRUCK DRIVER TASKS ANLYSIS DATA . . . . . . . 2-34

3.0 DRIVER WORKLOAD INDICATORS AND MODELS $\ldots \ldots \ldots \ldots \ldots \ldots \ldots .1$

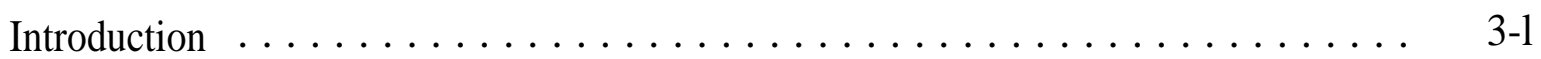

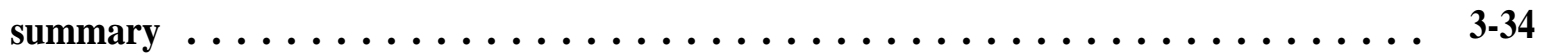

4.0 SAFETY RELEVANT CRITERIA … . . . . . . . . . . . . . . . . . . . . . . . . .

Introduction $\ldots \ldots \ldots \ldots \ldots \ldots \ldots \ldots \ldots \ldots \ldots \ldots \ldots \ldots \ldots \ldots$

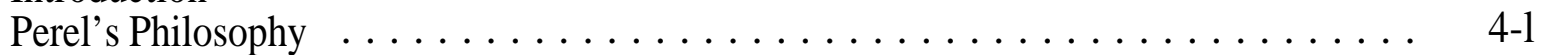

Visual Resource Parameters Approach $\ldots \ldots \ldots \ldots \ldots \ldots \ldots \ldots \ldots$. . . . . . . . . . . . . . . . . . . . . . . .

Harm's Concept . . . . . . . . . . . . . . . . . . . . . . . . . . . . . 4-3

Time-to-Line Crossing (TLC) Concept . . . . . . . . . . . . . . . . . . 4-3

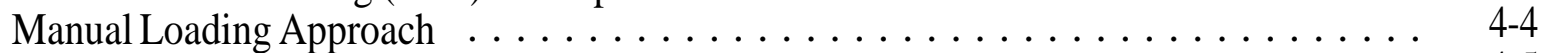

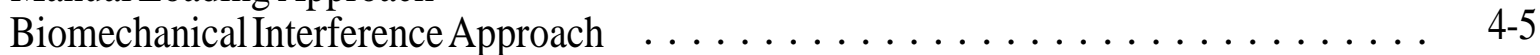

Other Resource Loading Approaches . . . . . . . . . . . . . . . . . . . $4-5$

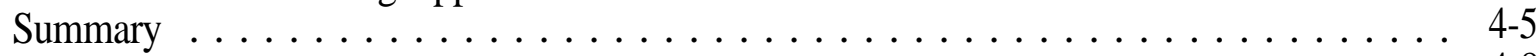

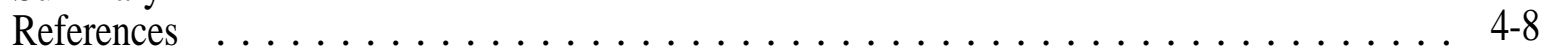

5.0 RISK TAKING AND WORKLOAD $\ldots \ldots, \ldots \ldots \ldots \ldots \ldots \ldots \ldots \ldots .1$

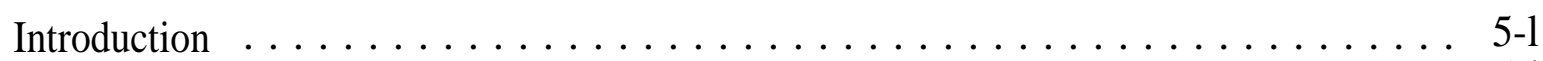

The Role of Risk in Models of Driving Behavior . . . . . . . . . . . . . . 5-1

Gaps in the Data Base $\ldots \ldots \ldots \ldots \ldots \ldots \ldots \ldots \ldots \ldots \ldots \ldots \ldots$

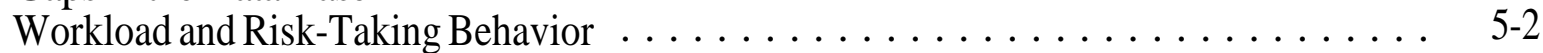

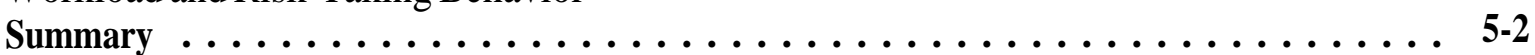

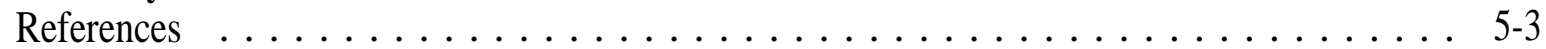




\section{TABLE OF CONTENTS}

(Continued)

Page

6.0 DRIVING TASK ANALYSIS DATA COLLECTION PROTOCOLS . . . . , . . . . 6-1

Activity Analysis/Work Sampling $\ldots \ldots \ldots \ldots \ldots \ldots \ldots \ldots \ldots$ 6-1

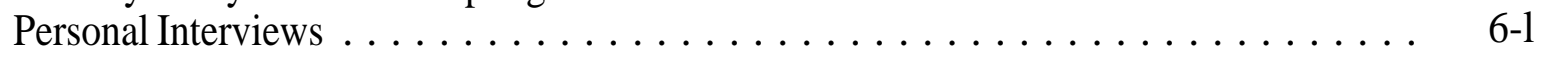

Commentary Driving/Protocol Analysis $\ldots \ldots \ldots \ldots \ldots \ldots \ldots \ldots \ldots$. . . . . . . . . . . .

Critical Incident Technique/Critical Decision Method . . . . . . . . . . . . . . . 6 6-4

Subjective Workload Ratings . . . . . . . . . . . . . . . . . . . 6-5

Visual Allocation ............................ 6-5

On-The-Road Driver Performance Monitoring $\ldots \ldots \ldots \ldots \ldots \ldots \ldots \ldots$ 6-5

Criticality Ratings and Rankings $\ldots \ldots \ldots \ldots \ldots \ldots \ldots \ldots \ldots \ldots \ldots \ldots$ 6 . $6 \ldots \ldots$

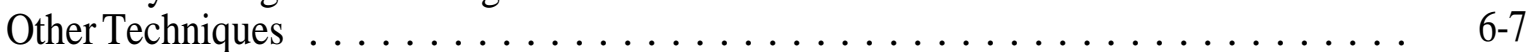

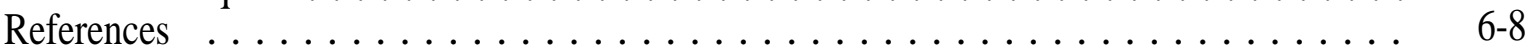

7.0 TASK 3 TASK ANALYSIS DATA COLLECTION PLAN $\ldots \ldots \ldots \ldots \ldots \ldots 7-1$

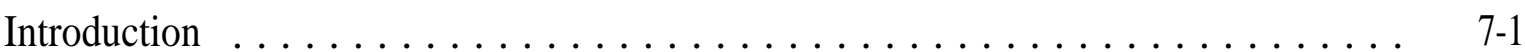

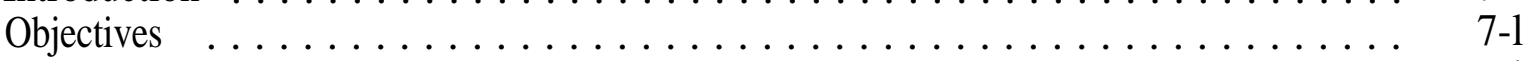

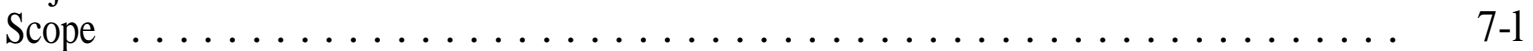

Approaches ............................... 7-2

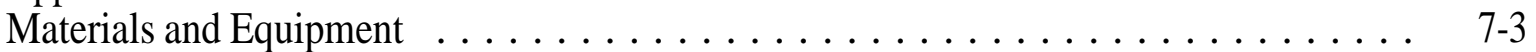

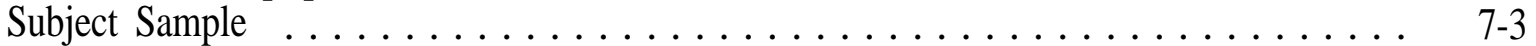

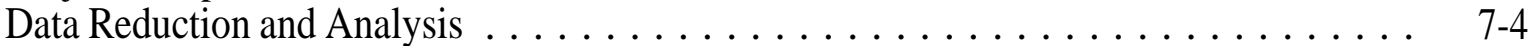

Anticipated Report Format for Task Analysis Data $\ldots \ldots \ldots \ldots \ldots \ldots \ldots \ldots$. . . . . . . . .

Task Analysis Data Collection Schedule . . . . . . . . . . . . . . . . . . . 7-4

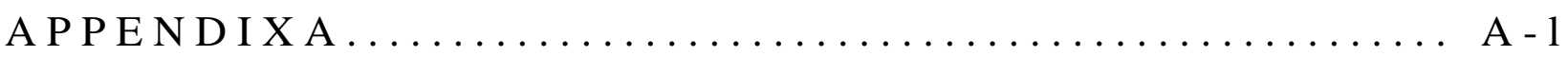

COGNITIVE TASK ANALYSIS METHODS $\ldots \ldots \ldots \ldots \ldots \ldots \ldots \ldots \ldots$ A-2

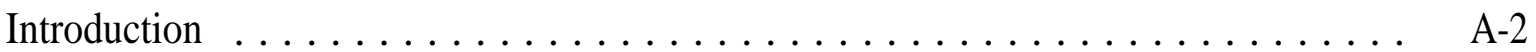

Cognitive Task Analysis Methods $\ldots \ldots \ldots \ldots \ldots \ldots \ldots \ldots \ldots \ldots \ldots \ldots \ldots$ A $\ldots \ldots \ldots$

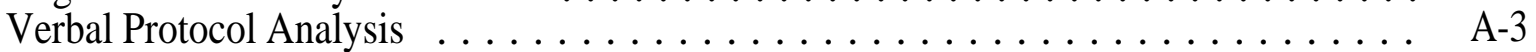

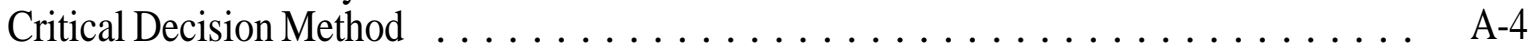

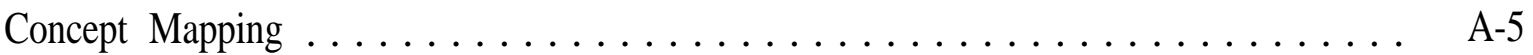

Conceptual Graphs ............................. A-6

Psychological Scaling Methods $\quad \ldots \ldots \ldots \ldots \ldots \ldots \ldots \ldots \ldots \ldots \ldots \ldots \ldots$ A-7

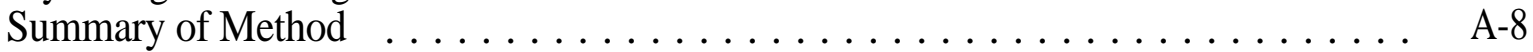

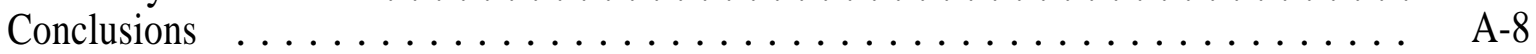

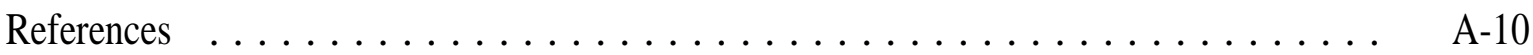




\section{LIST OF TABLES}

Page

TABLE 1.1. TASK ANALYSIS DATA AND PROTOCOLS REVIEW CRITERIA . . . . . . . 1-4

TABLE 1.2. CANDIDATE HEAVY VEHICLE DRIVER OPERATIONAL/VALIDITY

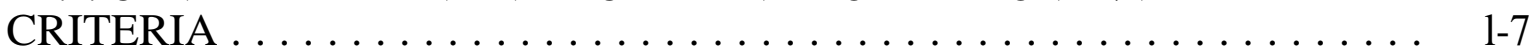

TABLE 2.1 INFORMATION CATEGORIES USED IN THE DRIVING BEHAVIOUR TASK

ANALYSIS . . . . . . . . . . . . . . . . . . . . 2-3

TABLE 2.2. LISTING OF STANDARD HEAVY VEHICLE TASKS AND SUB-TASKS TO BE CONSIDERED IN LONG-HAUL TRUCK DRIVING . . . . . . . . . . . . . 2-6

TABLE 2.3. TRUCK AND BUS DRIVER TASK LISTING $\ldots \ldots \ldots \ldots \ldots \ldots \ldots .2-10$

TABLE 2.4 SELECTED TRUCK AND BUS DRIVER TASK ANALYSIS $\ldots \ldots \ldots \ldots$. 2-17

TABLE 2.5. PTDIA DRIVER CURRICULUM $\ldots \ldots \ldots \ldots \ldots \ldots \ldots \ldots \ldots \ldots \ldots \ldots \ldots$

TABLE 2.6 DRIVER EDUCATION TASK ANALYSIS $\ldots \ldots \ldots \ldots, \ldots, \ldots, \ldots, 2-26$

TABLE 2.7 TASK LISTINGS AND ANALYSIS FOR SKID CONTROL . . . . . . . . 2-27

TABLE 2.8. DRIVER TASKS FOR INTERSECTION NEGOTIATION, AND LEAD TIME

DATA FOR LANE CHANGE AND SPEED CHANGE MANEUVERS . . . . . . . 2-31

TABLE 3.1. DRIVER LOADS (ESTIMATES) ASSOCIATED WITH SELECTED IN-CAB

TR U C K D E V I C E S . . . . . . . . . . . . . . . . . . 3 - 3

TABLE 3.2. IN-CAB DISPLAY \& CONTROL DEMANDS AS A FUNCTION OF

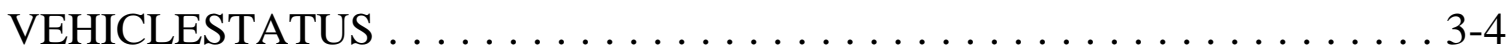

TABLE 3.3. TAXONOMIC DESCRIPTION OF INTERACTION WITH CONVENTIONAL

IN-CABDEVICES . . . . . . . . . . . . . . . . . . . . 3-9

TABLE 3.4. VEHICLE CONTROL TASK ANALYSIS ............... 3-13

TABLE 3.5. DIRECTIONAL INFORMATION NEEDS $\ldots \ldots \ldots \ldots \ldots \ldots \ldots \ldots$ 3-14

TABLE 3.6 DRIVING PERFORMANCE MEASURES ............... 3-30

TABLE 6.1. ACTIVITY SAMPLING SHEET USED FOR LONG-HAUL DRIVER TASK

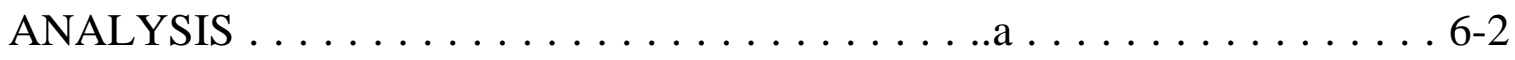

TABLE 6.2. INTERVIEW DATA FROM LONG-HAUL DRIVER TASK ANALYSIS . . . . . 6-3 


\section{LIST OF TABLES}

(Continued)

Page

TABLE 6.3. EXAMPLES OF WORKLOAD COMPONENTS SUITES ...........6-6

TABLE 7.1, DATA GATHERING PROTOCOL FOR NHTSA HV TASK ANALYSIS: INCAB

DEVICE

INTERACTION

7-5 


\section{LIST OF FIGURES}

page

FIGURE 3.1. EXAMPLE TASK SEQUENCE WITH IN-CAB TRIP RECORDER $\ldots \ldots$. . . 3-2

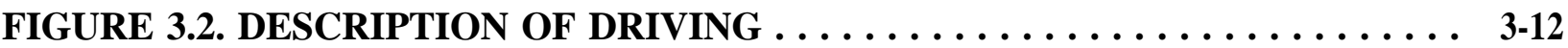

FIGURE 3.3. DRIVING PERFORMANCE DATA . . . . . . . . . . . . . . 3-17

FIGURE 3.4. MODEL OF INDIVIDUAL BEHAVIOR IN THE FACE OF DANGER . . . . 3-20 
NHTSA HEAVY VEHICLE DRIVER WORKLOAD ASSESSMENT

TASK 1 INTERIM REPORT: TASK ANALYSIS DATA \& PROTOCOLS REVIEW

to

NHTSA

January 31,1992

\subsection{BACKGROUND}

\section{$\underline{\text { Introduction }}$}

Standard practice in human factors engineering is to conduct a task analysis which describes the human activities associated with the area of interest. In the present case, the area of interest is heavy vehicle operation. The intent of this project is to develop a workload assessment protocol with which to evaluate the effects of high technology in-cab devices on driver performance and highway safety. Thus, a review of heavy vehicle task analysis data and protocols was conducted. This report contains the results of that review.

\section{Scope}

The scope of this Task 1 effort was to review available task analytic data and protocols pertinent to heavy vehicles and determine the availability and relevance of this data to the current effort. An attempt was made to operationally define 'pertinence' and 'relevance' in terms of criteria to be used for this review. The criteria used for this review are included later in this section and later sections reflect their application as appropriate.

The present review is not, nor was it intended to be, an exhaustive review of workload measurement and other relevant research. This will be the topic of Task 4. On the other hand, the Battelle staff began to review documents which bear upon the issue of driver workload induced by driving and auxiliary tasks and these reviews are included in this report. The Task 4 interim report will provide a more in-depth review of workload measurement in general and driver workload assessment in particular. More critical analyses will be provided on the methods and measures referred to here. At this point, none of the techniques of workload measurement have been critically assessed.

The Task 1 review also had to address two issues beyond task analysis per se. One was the development of safety relevant criteria for determining the point at which safety may be compromised for various driving conditions. A second issue was to address risk taking behavior and how it varies as a function of workload. These two topics are discussed in separate sections of this report. 


\section{Literature Search}

An extensive literature search was conducted by NHTSA using search terms for task analysis, driver models, and driver job and task analysis. Representative terms used in the searches included the following:

Task Analysis

Job Analysis

JTA

Activity Analysis

Timeline Analysis

Human Abilities

Task and Skill Analysis

Event Description

Event Analysis

Driver Models

Various vehicle search terms

NTIS, NHTSA's database, Psych Abstracts, and DTIC databases were searched. The returns were reviewed by Battelle staff and selected references were requested. Most of these references were provided to Battelle by the NHTSA COTR. Some items were ordered through NTIS, some were secured through the local library resources in Columbus, Ohio, and some were foreign materials which were requested by personal letter faxed to a representative from the organization or country of interest. Many materials were thus reviewed. Certain sources, though apparently relevant from their abstracts, proved to be of little use and are not included in this report. Some references which were ordered (or requested) could not be obtained and so are not included in this review. It is also possible that some materials will arrive after this report is due and such materials will be incorporated into subsequent tasks of this project.

\section{Criteria for Review}

This project posed an interesting challenge in that task analysis to support workload protocol development has never been accomplished, to our knowledge. Therefore, the criteria presented in Tables 1.1 and 1.2 were developed by the project staff in order to provide direction in this regard. These criteria were reviewed with the NHTSA COTR for completeness and correctness. Project staff were asked, for each source to be reviewed, to consider these criteria and assess the "usefulness" of the data presented. Here "usefulness" refers to the degree that a publication presents data which meet those criteria used in Tables 1.1 and 1.2, i.e., the degree to which that data can be used later to develop workload protocols to evaluate in-cab devices.

\section{Organization of this Report}

This Task 1 Interim Report is arranged in sections. Section 2.0, Task Descriptions and Task Criticality, provides a listing of the tasks which make up heavy truck driving. They represent verbal descriptions of the primary activities in which the driver engages while on the road. These tasks 
provide the backdrop against which in-cab device interaction will be placed These tasks provide the starting point for analysis of driver workload and the development of 'standard driving tasks' useful for workload assessment protocols.

Section 3.0, Driver Workload Indicators and Models, provides a preliminary look at methods and measures which have been applied to driver workload. This type of information was sought out because, in order to develop a workload assessment protocol, one needs at least a preliminary appreciation for what driver workloads are (see also Section 7.0). The literature reviewed is only a sample but it indicates several salient points. First, while there are a number of references addressing car driver workload, there are very, very few which address heavy vehicle driver workload. Second, a variety of methods have been applied to measure workload and these methods deserve close scrutiny in Task 4. Third, selected driver models provide some insights into the fine structure of driving tasks and so may be useful for workload assessment protocol development.

Section 4.0, Safety Relevant Criteria, addresses the issue of what candidate workload measures address highway safety. This discussion focusses on a selected measures and methods; the strengths and limitations of each are presented. These methods and measures, together with others not discussed in this report, will be presented and critically assessed in Task 4.0.

Section 5.0, Risk Taking and Workload, provides a review of the literature on risk taking and workload. NHTSA has expressed interest in the relationship between these two factors and the literature reviewed indicates the extent to which such a relationship has been described.

Section 6.0, Workload Assessment Methods and Protocols, reviews the various methods which have been applied to studies of (car) drivers. It reviews strengths and weaknesses of the various methods, provides some examples, and indicates the extent to which particular methods may be of use to the current effort.

This report culminates in Section 7.0, a preliminary Task Analysis Data Collection Plan for use in Task 3. The purpose of this plan is to describe a means by which the Battelle project staff will collect data useful for workload assessment protocol development and verify data culled from the sources described earlier in this report. This plan is subject to review and revision, of course, but it represents a viable approach to collecting data which will be of help in subsequent phases of this project. 


\section{TABLE 1.1. TASK ANALYSIS DATA AND PROTOCOLS REVIEW CRITERIA}

\begin{tabular}{|c|c|c|}
\hline Criteria & Description & Rationale \\
\hline Data Type: & $\begin{array}{l}\text { Indicate the Kind of data a source provides, e.g., } \\
\text { - on-the-road, } \\
\text { - drivingsimulator, } \\
\text { - analytical model, } \\
\text { - laboratory analogue to driving, } \\
\text { - armchair analysis by task analyst or driver SME. }\end{array}$ & $\begin{array}{l}\text { The nature of a source will determine the extent to which } \\
\text { some other criteria are applicable. For example, } \\
\text { operationalvalidity is likely to be moot for an armchair } \\
\text { analysis. Similarly, environmental conditions likely will } \\
\text { not apply, or will apply only indirectly, to driving } \\
\text { simulator results (unless an advanced simulator or one with } \\
\text { specific environmental simulation capabilities was used). }\end{array}$ \\
\hline Task Listing: & $\begin{array}{l}\text { List, in a table or appendix, the tasks indicated in a source. If } \\
\text { possible, include if a task is a: } \\
\text { - primary driving task (lane keeping, speed maintenance), } \\
\text { - ancillary task (communications, HVAC adjustment), or } \\
\text { - additional driver behsvion (e.g., pouring coffee, lighting } \\
\text { a cigarette). } \\
\text { Specific dats on high technology device interactions would be } \\
\text { particularly useful. }\end{array}$ & $\begin{array}{l}\text { This listing is the basis for assessing criticality and gaining } \\
\text { a better understanding of the driving situation.. }\end{array}$ \\
\hline Task Structure: & $\begin{array}{l}\text { Does a source indicate the sequential and simultaneous ordering of } \\
\text { subtasks? A diagram should be included if available. }\end{array}$ & $\begin{array}{l}\text { The detailed structure of a task may reveal bottlenecks or } \\
\text { sources of conflict with a subsidiary task. }\end{array}$ \\
\hline $\begin{array}{l}\text { Driver } \\
\text { Descriptions: }\end{array}$ & $\begin{array}{l}\text { What is the nature of the driver population used in the source? What } \\
\text { strategies are they reported to exhibit to deal with increased task } \\
\text { demands? }\end{array}$ & $\begin{array}{l}\text { It is assumed that workload and strategies for task } \\
\text { completion will be, to at least some extent, a function of } \\
\text { driver characteristics such as age, experience, sex } \\
\text { (perhaps), cognitive style (perhaps), etc. }\end{array}$ \\
\hline $\begin{array}{l}\text { Timeline } \\
\text { Analysis: }\end{array}$ & $\begin{array}{l}\text { To what extent does a source indicate the distribution of tasks over a } \\
\text { haul scenario? }\end{array}$ & $\begin{array}{l}\text { In addition to criticality, frequency of occurrence of a task } \\
\text { may be useful in developing workload assessment } \\
\text { protocols which are tailored for maximum efficiency and } \\
\text { effectiveness or are appropriate to a particular phase of } \\
\text { heavy vehicle operation. }\end{array}$ \\
\hline Safety Criticality: & $\begin{array}{l}\text { To what extent does the source indicate what tasks are critical from a } \\
\text { safety standpoint, i.e., driving tasks which are not very tolerant of } \\
\text { intrusion. Criticality as a function of driving conditions will also be } \\
\text { useful. Indicate the means by which criticality was assessed. }\end{array}$ & $\begin{array}{l}\text { The SOW/contract indicates a need to 'delineate a set of } \\
\text { safety-critical driving tasks that can be used to assess the } \\
\text { impact of applying new technologies withii heavy } \\
\text { vehicles" (p. 9). }\end{array}$ \\
\hline $\begin{array}{l}\text { Driver Information } \\
\text { Needs: }\end{array}$ & $\begin{array}{l}\text { To what extent does the source indicate data like the following for } \\
\text { tasks and subtasks: information required, initiating cues, } \\
\text { information format/modality, feedback, terminating cues, feedback } \\
\text { formatmodality, decision processes, actions taken/outputs generated. } \\
\text { These fall within the framework of classical task analysis. }\end{array}$ & $\begin{array}{l}\text { The SOW/contract indicates that such data will be } \\
\text { collected as part of Task } 3 \text {. Competition among } \\
\text { information channels/modalities is of importance for } \\
\text { workload assessment. }\end{array}$ \\
\hline $\begin{array}{l}\text { Operational } \\
\text { Validity: }\end{array}$ & $\begin{array}{l}\text { Determine the operational validity of existing task analysis data using } \\
\text { Table } 1 \text { criteria. Note if source provides empirical, analytical, or } \\
\text { qualitative results based on abstract analysis of the driving tasks. }\end{array}$ & $\begin{array}{l}\text { The SOW/contract states that we should determine "the } \\
\text { validity of data for current heavy vehicle configurations } \\
\text { and tasks " (p. 10). Furthermore, it is stated that "these } \\
\text { criteria shall specifically address the issue of changes that } \\
\text { have taken place in the "typical' heavy vehicle and/or } \\
\text { driving tasks since the original data was collected..." (p. } \\
\text { 11). }\end{array}$ \\
\hline $\begin{array}{l}\text { Driving } \\
\text { Conditions: }\end{array}$ & $\begin{array}{l}\text { Task workload is assumed to be shaped, at least in part., by tbe } \\
\text { driving conditions for a particular set of data. At a minimum, the } \\
\text { source should describe the following conditions: } \\
\text { - heavy/light traffic } \\
\text { - divided/undividedroadway } \\
\text { - good weather/poor weather } \\
\text { - day/night }\end{array}$ & $\begin{array}{l}\text { The SOW/contract states that task analysis data reported in } \\
\text { a source will be carried out under the conditions listed } \\
\text { above, at a minimum (p. 11). Task workload is } \\
\text { modulated, at least to some extent, by the driving } \\
\text { conditions, as mentioned above. }\end{array}$ \\
\hline
\end{tabular}




\begin{tabular}{|c|c|c|c|}
\hline Criteria & & Description & Rationale \\
\hline $\begin{array}{l}\text { Criteria for } \\
\text { SafetyThresholds: }\end{array}$ & \multicolumn{2}{|c|}{$\begin{array}{l}\text { To what extent does a source indicate performance or measurement } \\
\text { tolerances beyond which safety is compromised? What is the } \\
\text { reported rationale behind these 'redlines*? Examples may include } \\
\text { allowable lane drift, minimum time headway, maximum visual } \\
\text { glance duration, maximum number of glances per unit time, etc. } \\
\text { Battelle shall suggest a method for determining criteria and consider } \\
\text { the development of a matrix specifying the criteria for the various } \\
\text { dependent measures and driving conditions. These criteria and their } \\
\text { bases shall be developed in consultation with the COTR and will be } \\
\text { included in the interim report. }\end{array}$} & $\begin{array}{l}\text { The SOW/contract states that as part of this effort, "the } \\
\text { contractors shall develop criteria for determining the point } \\
\text { at which safety may be compromised for tbe various } \\
\text { driving conditions being evaluated" (p. 11). }\end{array}$ \\
\hline $\begin{array}{l}\text { Data on Driver } \\
\text { Loading } \\
\text { and Usefulness } \\
\text { for Current Effott: }\end{array}$ & $\begin{array}{l}\text { Determine us } \\
\text { effort, i.e., to } \\
\text { assessmentpr } \\
\text { information s } \\
\text { • sc } \\
\text { de } \\
\text { su } \\
\text { - pa } \\
\text { Sp } \\
\text { th } \\
\text { se } \\
\text { At a minimu } \\
\text { indications fr } \\
\text { visual load: } \\
\text { manual load: } \\
\text { An indicatio } \\
\text { types is give } \\
\text { Manual only } \\
\text { pressing set } \\
\text { without furth } \\
\text { volume, char }\end{array}$ & $\begin{array}{l}\text { ness of existing task analysis data for use in this } \\
\text { lopment a heavy vehicle driver workload } \\
\text { ol. At a minimum such task descriptions and } \\
\text { d give the following: } \\
\text { indication of the attentional(read "workload") } \\
\text { ds of the tasks such as "time to complete a task } \\
\text { sfully", and } \\
\text { de an analytical basis for determining tbe spare } \\
\text { ty of the driver (perceptual, cognitive, motor). } \\
\text { capacity is defined in the contract in terms of TIME } \\
\text { ver's attention can be taken away from the primary } \\
\text { task(s) without compromising safety (for a given } \\
\text { onditions); } \\
\text { is recommended that source be reviewed for any } \\
\text { he following: } \\
\text { measured by glance duration, glance frequency, } \\
\text { etc. } \\
\text { measured by time the hand is off the wheel, } \\
\text { increased lane keeping variability, etc.; } \\
\text { measured by commentary driving, secondary task } \\
\text { results, delayed responding, etc; } \\
\text { measured by number of messages, message length, } \\
\text { etc. } \\
\text { mew: } \\
\text { measured by percent time use of tight foot, } \\
\text { percent time use of left foot, etc.l } \\
\text { g falion reference, e.g., turning on radio, adjusting } \\
\text { fan speed on air conditioner). }\end{array}$ & \\
\hline
\end{tabular}




\begin{tabular}{|c|c|c|}
\hline II Criteria & Description & Rationale \\
\hline & $\begin{array}{l}\text { Vii only (tasks that are completely visual, e.g., reading } \\
\text { speedometer, determining current mode of HVAC, determining from } \\
\text { a map display whether vehicle is headed in correct direction) } \\
\text { Vii Primarily (some manual input requited, e.g., determining the } \\
\text { station frequency on the radio then display is initially in time mode, } \\
\text { changing modes and verifying change and then completing the task). } \\
\text { Vii-manual (tasks with interactive visual and manual } \\
\text { demands, e.g., manually tuning a radio to a specific frequency, } \\
\text { operating a cellular telephone, making mirror adjustments, zooming } \\
\text { in or out on a map display) } \\
\text { Note that this taxonomy was taken from a paper which emphasized } \\
\text { visual and manual loading. Other task types are possible (e.g., } \\
\text { auditory feedback when adjusting radio volume). }\end{array}$ & $\begin{array}{l}\text { The SOW/contract states that "risk taking behavior' and } \\
\text { how it varies as a function of driving condition and } \\
\text { workload is of particular interest to NHTSA" (p. 11). }\end{array}$ \\
\hline $\begin{array}{l}\text { Risk Taking } \\
\text { Behavior: }\end{array}$ & $\begin{array}{l}\text { Does the source indicate any risk taking behavior and how it varies } \\
\text { as a function of driving conditions and workload? }\end{array}$ & \\
\hline Methodology: & $\begin{array}{l}\text { Provide a synopsis of what methods were used in a task analysis. } \\
\text { This may, across different sources, include methods for identifying } \\
\text { tasks, methods for determining safety criticality or workload ratings, } \\
\text { and methods of assessing driver performance. }\end{array}$ & $\begin{array}{l}\text { Methodological approaches will be important in structuring } \\
\text { the Task } 3 \text { task analysis data collection plan. } \\
\text { Note that we are interested in both driving tasks and in } \\
\text { interactions with high-technology in-cab devices. It is } \\
\text { unlikely that device task analyses are currently available. }\end{array}$ \\
\hline
\end{tabular}




\section{TABLE 1.2. CANDIDATE HEAVY VEHICLE DRIVER OPERATIONAL/VALIDITY CRITERIA}

\begin{tabular}{|c|c|}
\hline Candidate Criteria & Changes Over the Last $10-20$ Years \\
\hline \multicolumn{2}{|l|}{ Equipment $\mathrm{Cr}$ i t e r i a } \\
\hline Truck Length & $\begin{array}{l}\text { U.S. truck trailers are longer now ( } 48 \text { and } 53 \text { feet now vs. } \\
40 \text { and } 45 \text { feet } 10 \text { years ago). }\end{array}$ \\
\hline Truck Width & Trucks are wider now (102" now vs. 96" then). \\
\hline Truck Engines & $\begin{array}{l}\text { Truck engines are more powerful and efficient. The driver } \\
\text { can now sustain higher speeds and use less shifting. }\end{array}$ \\
\hline $\begin{array}{l}\text { Electronics and } \\
\text { Electromechanical Devices }\end{array}$ & $\begin{array}{l}\text { Electronics and Electromechanical devices are replacing } \\
\text { hydraulic/mechanical control systems of the engine and drive } \\
\text { train. }\end{array}$ \\
\hline \multicolumn{2}{|l|}{ Environmental 'Criteria } \\
\hline Traffic & There are many more cars and trucks on the road now. \\
\hline Construction & $\begin{array}{l}\text { Due to the deterioration of the U.S. highway system, there is } \\
\text { more construction now, presenting additional driving hazards. }\end{array}$ \\
\hline Speed & $\begin{array}{l}\text { Maximum allowable speeds are higher now than during the } \\
\text { oil crisis of the late } 70 \text { s and early } 80 \text { s. }\end{array}$ \\
\hline \multicolumn{2}{|l|}{ Driver Criteria } \\
\hline Age & $\begin{array}{l}\text { Some think that there are two kinds of driver: the young } \\
\text { "cowboy" risk-taker and the older "road knight". Driving } \\
\text { experience and perceptual acuity are age-dependent to some } \\
\text { extent. }\end{array}$ \\
\hline Fitness for Duty & $\begin{array}{l}\text { Driver performance will be affected by such things as drug } \\
\text { use and fatioue. }\end{array}$ \\
\hline Independents & $\begin{array}{l}\text { tndependent truckers may be more likely to engage in risky } \\
\text { driving than drivers from larger, more established firms. }\end{array}$ \\
\hline Attitudes & $\begin{array}{l}\text { Drivers today feel more over-regulated than their counter- } \\
\text { parts of } 10-20 \text { years ago. Since the } 1980 \text { economic dereg- } \\
\text { ulation of the trucking industry, truckers feel they are being } \\
\text { pressured to move a lot more freight for less pay due to the } \\
\text { competition. }\end{array}$ \\
\hline \multicolumn{2}{|l|}{ Prócedural Criteria } \\
\hline Just-in-Time Scheduling & $\begin{array}{l}\text { Current scheduling and dispatching practices tend to place } \\
\text { more time stress on drivers. }\end{array}$ \\
\hline
\end{tabular}




\subsection{DRIVING TASKS AND TASK CRITICALITY}

\section{$\underline{\text { Introduction }}$}

Task listings are useful for understanding what activities and driver behaviors are involved in heavy vehicle operation. Literature searches revealed listings of truck driver tasks in only a few sources which came from Canada, Sweden, and the United States. In addition, there were task analysis listings for car driving which we believe have relevance to our project objectives. In reviewing these sources and preparing the tables to be presented here, it has been our assumption that our concerns for this project require us to focus on driver tasks in which the vehicle is in motion. Therefore, tasks have been selected for inclusion here based on that assumption. Various task listing sources are presented below.

Rabideau, G., \& Young, P. (1973). Identification of safety-critical driving behaviors by means of task analysis (pp. 26 - 34). Proceedings of the Scientific Session of the 10th Annual Meeting of Traffic Iniurv Research Foundation of Canada.

Data Type:

Task Listing:

Task Structure:

Driver:

Timeline

Analysis:

Driver Information Needs:
Formal task analysis. Driving was conceived of as a continuous feedback skill with some often repeated discrete subtasks treated as procedures.

As part of a research program supported by the National Research Council (Canada), Rabideau and Young used traditional task analysis for analysis of the two major driver functions:

1) maintaining appropriate forward motion, and

2) maintaining appropriate path and direction of motion.

None.

It was mentioned that the analysis included in the report benefitted from inhouse consultation with persons who have had considerable truck driving experience.

None.

See Table 2.1; it contains a rich description of the information requirements associated with maintaining forward motion and path within posted speed limits, the only two major functions considered. The task analysis does not explicitly include perceptual, cognitive, or motor loadings, Nor does it provide any explicit timeline or timing information.

Operational Validity:

Moderate, based on the reference to experienced driver consultants. 
Driving

Conditions:

Criteria for

Safety Thresholds:
Ideal-case conditions for data in Table 2.1 (straight road, no traffic), with negative, positive, and level road grades.

Usefulness

of Data:

Provides details of information requirements for truck driving tasks. May serve as the 'baseline' from which deviations in driver information needs are noted,

Risk Taking

Behavior: None indicated.

Reviewer: $\quad$ L. Tijerina 


\section{TABLE 2.1 INFORMATION CATEGORIES USED IN THE DRIVING}

BEHAVIOUR TASK ANALYSIS (SOURCE: RABIDEAU \& YOUNG, 1973).

\begin{tabular}{|c|c|}
\hline Category & Example \\
\hline TASK IDENTIFICATION & Maintain required forward motion and path within posted speed. \\
\hline $\begin{array}{l}\text { DISPLAY } \\
\text { Problem }\end{array}$ & $\begin{array}{l}\text { drive truck at speed limit on straight mad } \\
\text { assume no curves or traffic } \\
\text { assume level, positive, and negative roadway grades }\end{array}$ \\
\hline CriticalStimulus Variables & $\begin{array}{l}\text { speedlimit } \\
\text { grade of road (present and approaching) } \\
\text { engine speed and characteristics } \\
\text { loading of vehicle } \\
\text { position of accelerator pedal } \\
\text { gear in use and characteristics } \\
\text { camber in road surface } \\
\text { obstacles on roadway }\end{array}$ \\
\hline Time Values & $\begin{array}{l}\text { steering corrections before truck leaves lane (dependent on speed and yaw angle) } \\
\text { other times, e.g., optimum shift points, optimum braking times when over speed } \\
\text { limit,notsafety-critical }\end{array}$ \\
\hline Display Noise & $\begin{array}{l}\text { poor visibility (includes sun glare) } \\
\text { unknown speed limit } \\
\text { awkward or heavy boots } \\
\text { "deadband" in steering mechanism }\end{array}$ \\
\hline REQUIRED DECISIONS & $\begin{array}{l}\text { speed up or slow down now } \\
\text { speed up or slow down soon } \\
\text { move steering wheel }\end{array}$ \\
\hline CONTROLS & $\begin{array}{l}\text { 1) accelerator pedal, 2) brake pedal, } \\
\text { 3) clutch pedal, 4) gear selector, } \\
\text { 5) steering wheel }\end{array}$ \\
\hline CONTROL ACTIVATION & $\begin{array}{l}\text { 1) move up or slow down to position yielding desired speed/acceleration } \\
\text { 2) push down to produce desired deceleration } \\
\text { 3)-4) described in shifting procedure } \\
\text { 5) turn in direction to be taken, then straighten out }\end{array}$ \\
\hline CONTROL ACMON & $\begin{array}{l}\text { 1) determined by acceleration/deceleration propertiesof truck for particular gear, } \\
\text { grade and loading } \\
\text { 2) rate of deceleration dependent upon (a) grade, (b) speed, (c) vehicle weight, } \\
\text { (d) force on pedal } \\
\text { 3) (a) amount of rotation depends on steering ratio of vehicle } \\
\text { (b) force depends on ratio, loading, amount of power assist, road surface, } \\
\text { speed, and tire pressure }\end{array}$ \\
\hline $\begin{array}{r}\text { FEEDBACK } \\
\text { Cues }\end{array}$ & $\begin{array}{l}\text { visual and auditory sensations of speed change } \\
\text { speedometerreading changes }\end{array}$ \\
\hline Time Delay & $\begin{array}{l}\text { virtually no time delay to onsets of change } \\
\text { completion time varies with conditions }\end{array}$ \\
\hline Criteria of Response Adequacy & $\begin{array}{l}\text { adequacy indicated by speedometer reading same as posted limit } \\
\text { also vehicle perceived as centered in own lane }\end{array}$ \\
\hline CriticalValues & Corrective Actions \\
\hline $\begin{array}{l}\text { stalledengine } \\
\text { locked brakes } \\
\text { "missed" shift } \\
\text { wheels out of lane }\end{array}$ & $\begin{array}{l}\text { restatt (procedure) } \\
\text { releasepedalpressure } \\
\text { repeat (shifting procedure) } \\
\text { turn steering wheel in smooth motion as required to correct lateral position }\end{array}$ \\
\hline
\end{tabular}




\begin{tabular}{||c|c||}
\hline \hline \multicolumn{1}{|c||}{ Category } & Example \\
\hline CHARACTERISTIC ERRORS & \\
"hunting" around speed limit due to \\
acceleratorovercorrection \\
weaving down road due to steering \\
overcorrection \\
excessive frequencies of change in: \\
$\begin{array}{l}\text { (1) acceleration (increases fuel } \\
\text { consumption) } \\
\text { (2) braking (increases break wear) }\end{array}$ \\
\hline \hline
\end{tabular}


Rabideau. G.. \& Young. P. (1973). Effects of long-duration driving on performance of safetycritical tasks. Waterloo: University of Waterloo.

Rabideau, G., \& Young, P. (1974). Validation of a task analvsis methodology applied to longhaul truck driver behaviour. National Research Council of Canada. (Microfiche No. ED 083 295).

(Note: $\quad$ These two sources provide essentially the same task analytic data).

Data Type: $\quad$ Formal analysis, interviewing of truck driver sample, on-the-road observation.

Task Listing: See Table 2.2. It is particularly noteworthy that the authors initially omitted some off-road activities from further analysis, presumably because they identified safety-criticality in only terms of conditions when the vehicle was in motion. The authors also adopted an event-based mission approach rather than time- or distance-based mission approach because of the highly variable driving conditions. The tasks given in table 2.2 were selected on the basis of the following:

criticality to driving safety

susceptibility to fatigue degradation

predictability or the inverse of the proportion of occurrences that a given task is performed on a contingency or emergency basis.

Task Structure: None.

Driver: $\quad$ A number (unspecified) of professional truck drivers were questioned in structured interviews.

Timeline

Analysis: $\quad$ None.

Driver Information

Needs:

Table 2.1 was also presented in these two reports.

Operational

Validity:

Moderate to high. The authors used the following methods to validate a preliminary task analysis, the results of which are Table 2.2:

- $\quad$ Structured checklists and interviews: Personal data (age, sex, driving experience) were collected. Interviews covered 10 topics: general safe driving; risk avoidance in unfamiliar situations; other vehicle behaviors; day versus night driving; visual search technique; critical incident causes; route pacing; rest stop criteria (timing); mechanical failures in truck; vehicle characteristics (subdivided into 6 topics: braking, steering and handling, transmission and shifting, instrumentation, small controls). 
TABLE 2.2. LISTING OF STANDARD HEAVY VEHICLE TASKS AND SUB-TASKS TO BE CONSIDERED IN LONG-HAUL TRUCK DRIVING

(SOURCE: RABIDEAU AND YOUNG, 1974).

\begin{tabular}{|c|c|}
\hline Task & Subtask \\
\hline $\begin{array}{l}\text { 1. Maintain forward vehicle motion within } \\
\text { a) speed limit } \\
\text { b) directional constraints }\end{array}$ & $\begin{array}{l}\text { 1.1 Control center-lane tracking } \\
1.2 \text { Adjust vehicle speed }\end{array}$ \\
\hline $\begin{array}{l}\text { 2. Maintain forward vehicle motion within } \\
\text { a) safety limit } \\
\text { b) directional constraints }\end{array}$ & $\begin{array}{l}\text { 2.1 Control center-lane tracking } \\
\text { 2.2 Adjust vehicle speed }\end{array}$ \\
\hline $\begin{array}{l}\text { 3. Maintain forward vehicle motion on } \\
\text { highway shared with other vehicles }\end{array}$ & $\begin{array}{l}\text { 3.1 Control center-lane tracking } \\
\text { 3.2 Adjust vehicle speed } \\
\text { 3.3 Monitor other vehicle courses and positions }\end{array}$ \\
\hline $\begin{array}{l}\text { 4. Control acceleration during turns } \\
\text { a) cornering } \\
\text { b) entering highway } \\
\text { c) exiting highway }\end{array}$ & $\begin{array}{l}\text { 4.1 Control rate of turn } \\
\text { 4.2 Decelerate vehicle movement } \\
\text { 4.3 Accelerate vehicle movement } \\
\text { 4.4 Monitor other vehicle courses and positions } \\
\text { 4.5 Monitor fixed object locations }\end{array}$ \\
\hline $\begin{array}{l}\text { 5. Control acceleration during overtake and } \\
\text { passing } \\
\text { a) fixed aperture-no traffic } \\
\text { b) fixed aperture-traffic } \\
\text { c) variable aperture } \\
\end{array}$ & $\begin{array}{l}\text { 5.1 Control lane track of vehicle } \\
\text { 5.2 Adjust vehicle speed } \\
\text { 5.3 Monitor passing aperture } \\
\text { 5.4 Monitor other vehicle courses and positions }\end{array}$ \\
\hline $\begin{array}{l}\text { 6. Control acceleration during stopping } \\
\text { maneuvers } \\
\text { a) normal } \\
\text { b) emergency }\end{array}$ & $\begin{array}{l}\text { 6.1 Control center-lane tracking } \\
\text { 6.2 Decelerate vehicle movement } \\
\text { 6.3 Monitor available stopping distance } \\
\text { 6.4 Select "escape route" }\end{array}$ \\
\hline $\begin{array}{l}\text { 7. Control track during obstacle avoidance } \\
\text { a) fixed obstacle } \\
\text { b) moving obstacle }\end{array}$ & $\begin{array}{l}\text { 7.1 Control rate of turn } \\
\text { 7.2 Decelerate vehicle movement } \\
\text { 7.3 Accelerate vehicle movement } \\
\text { 7.4 Monitor relative position and course/rate if } \\
\text { moving }\end{array}$ \\
\hline
\end{tabular}


En route observations and activity analvsis: This was based on 20 second segments randomly selected for total of 60 such segments used per 8 hours of driving. Data collectors used a pre-established activity sampling sheet and the frequency of occurrence ranged from 1 to 60 in terms of number of segments during which activity took place. Other conditions, e.g., weather, traffic density, nature of the road segment, were noted but not included in tabled results.

Driver critical incidents: Drivers were encouraged to recall 'close calls', describe them and speculate on their causes.

Descriptions of these data collection protocols will be given in a later section of this report.

\section{Driving}

Conditions: None specified.

Criteria for

Safety Thresholds: None.

Usefulness

of Data:

\section{Risk Taking}

Behavior:
Provides a list of safety-critical tasks which may be a focus for the present effort. This work specifically focusses on long-haul truck drivers, making it particularly relevant to the heavy vehicle truck driver workload assessment project.

Rabideau and Young also analyzed safety critical behaviors which may affect driving safety positively (if carried out) or negatively (if omitted). Note that response or actions were not considered under the assumptions that experienced professional drivers will know what responses are appropriate and have the skill to make them. They presented the following examples of positive safety-critical behaviors in Sensing/Recognition and Decision Making:

Sensing and

recognition

-obstacles on the road

- air temperature falling below 32' F

- brake lights on forward vehicles

- loose gravel at corners

Decisions

- reduce speed during periods of uncertain visibility

- increase following distance on wet roads

- speed to enter curve

- rate of closure with oncoming vehicles during overtaking.

Negative safety critical behaviors are defined as the inverse of positive safety critical behaviors and are said to arise in two ways: a) omitting a $\mathrm{SCB}+$ behavior and 2) deliberately choosing an SCB- behavior. The first is likely if the driver fails to 
perceive or recognize hazards. For inexperienced drivers, they may perceive and recognize the hazard, but not know what an appropriate response is. Deliberately choosing SCB- behaviors might be due to a lack of experience, inaccurate recall of past experience, or incorrect stored information. Alternatively, the driver may have a (mistaken) subjective probability that he can make an SCB- maneuver and succeed.

Reviewer: L. Tijerina

\section{Moe, G. L, Kelley, G. R., \& Farlow, D. E. (1973). Truck and Bus Driver Task Analvsis (DOT HS 800 835). Goleta, CA: Human Factors Research, Inc.}

Data Type: Formal analysis based on data in handbooks, training manuals, and regulations plus driver expert inputs; validation of tasks through separate panel of driver expert judges; extensive on-the-road observations; interviews and ratings provided by a sample of drivers (see below).

Task Listing: See Table 2.3. These tasks were selected from the full report to indicate those tasks judged to be above-average in safety-criticality and/or represent tasks and subtasks in which the vehicle is in motion. The Moe et al. task analysis was carried out as an extension to the task descriptions described by McKnight and his colleagues for car driving (see below). This work was part of a larger effort to develop a battery of candidate knowledge test items for use in testing bus and truck drivers for licensing purposes. A prerequisite for this development was a detailed analysis and description of driver behaviors required for safe vehicle operation. Moe, et al.s task analysis was also described as being of use in training, evaluation, and licensing of truck and bus drivers. In all, 420 tasks and subtasks were identified.

Task Structure: $\quad$ None per se. Many of the subtasks read as though they are sequential in nature. However, this cannot be confidently inferred from a reading of the list alone.

Driver: $\quad$ Thirty-seven (37) truck driver Subject Matter Experts (SMEs) and 24 Bus Driver SMEs rated tasks on safety criticality. Each SME was sent three envelopes with 25 randomly selected task descriptions in each envelope. The SME first sorted the 25 task descriptions into three piles of safety criticality, i.e.,

High criticality - Tasks the driver must do Moderate criticality - Tasks the driver ought to do Low criticality - Tasks the driver may do

Then each SME rank ordered the 25 tasks from most safety-critical to least safetycritical. Finally, the SME drew a line to mark the High-criticality tasks from the Moderate-criticality task and the Moderate-criticality tasks from the Low-criticality tasks. This procedure was used to identify high criticality driver tasks. Results indicated there was considerable disagreement among SMEs on the rank position of the tasks but general agreement on criticality categories. That is, SMEs agreed more 
on what tasks are safety critical- and less on which of the tasks was most critical, second-most critical, third-most critical, etc.

Timeline

Analysis:

None. 
TABLE 2.3. TRUCK AND BUS DRIVER TASK LISTING (ADAPTED FROM MOE, KELLEY, AND FARLOW, 1973).

\begin{tabular}{|c|c|c|c|}
\hline \multicolumn{2}{|r|}{ Tasks } & \multicolumn{2}{|r|}{ Subtasks } \\
\hline I. & $\begin{array}{l}\text { Preoperative Procedures } \\
\text { A. Trip Planning }\end{array}$ & $\begin{array}{l}1 . \\
2 . \\
3 . \\
4 .\end{array}$ & $\begin{array}{l}\text { Determine destination } \\
\text { Determine cargo characteristics } \\
\text { Determine route } \\
\text { Obtain route approval from dispatcher }\end{array}$ \\
\hline & VehicleInspection & $\begin{array}{l}1 . \\
2 . \\
3 . \\
4 . \\
5 . \\
6 .\end{array}$ & $\begin{array}{l}\text { Before entering truck } \\
\text { Preliminarywalk-aroundinspection } \\
\text { Inspect engine compartment } \\
\text { Enter vehicle } \\
\text { Perform walk-around inspection } \\
\text { Reenter vehicle }\end{array}$ \\
\hline & $\begin{array}{l}\text { Three mile check (done during first few } \\
\text { minutes of driving }\end{array}$ & $\begin{array}{l}1 . \\
2 . \\
3 .\end{array}$ & $\begin{array}{l}\text { Engine and power train performance } \\
\text { Steering mechanism } \\
\text { Vehicle tracking }\end{array}$ \\
\hline II. & $\begin{array}{l}\text { Routine Driving Tasks } \\
\text { A. } \quad \text { Accelerating to Roadway Speed }\end{array}$ & 1. & Accelerate to maximum speed in each gear \\
\hline & Shifting Gears & 2. & \begin{tabular}{ll}
\multicolumn{2}{l}{ Standard transmission } \\
- & Double-clutch \\
- & Downshift
\end{tabular} \\
\hline & DirectionalControl & 1. & $\begin{array}{l}\text { Steering - General } \\
-\quad \text { Check trailer alignment using rear view mirrors to } \\
\text { determine if trailer is tracking properly } \\
\text { - } \\
\text { Prevent weaving by avoiding jerky control } \\
\text { Use cues from distant field of vision to anticipate required } \\
\text { steering responses and to avoid fishtailing }\end{array}$ \\
\hline & & 2. & 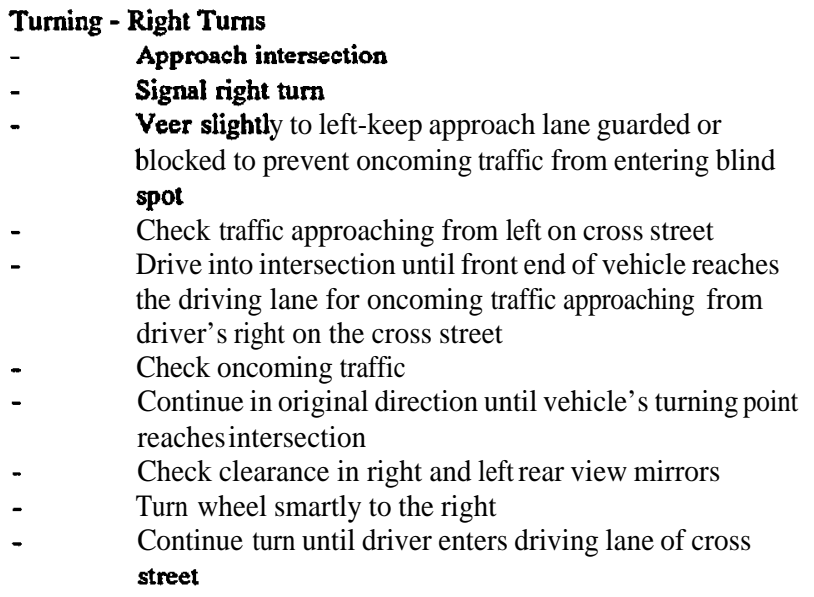 \\
\hline
\end{tabular}




\begin{tabular}{|c|c|c|c|}
\hline \multirow{2}{*}{\multicolumn{2}{|c|}{ Tasks }} & \multicolumn{2}{|r|}{ Subtasks } \\
\hline & & 3. & $\begin{array}{l}\text { Curves } \\
\text { Slow to speed limit posted for curves } \\
\text { Judge radius of curve } \\
\text { Select a turning radius appropriate for the curve } \\
\text { Steer to outside portion of lane } \\
\text { Check rear view mirror to ensure rear end of vehicle has } \\
\text { not drifted into adjacent lane on outboard side of curve } \\
\text { Judge correctness of speed and steering control and make } \\
\text { adjustments asnecessary } \\
\text { Upgrades } \\
\text { Keep well to the right (or in the right-hand lane of multi- } \\
\text { lane highway) } \\
\text { Do not pull off if shoulder is soft if covered with loose } \\
\text { dirt which could cause a dust cloud, or if driving } \\
\text { conditions are bad }\end{array}$ \\
\hline & & 5. & $\begin{array}{l}\text { Downgrades } \\
\text { Stop and inspect braking system and tires before starting } \\
\text { down long/hazardous hills } \\
\text { Approach top of grade at slow speed } \\
\text { Keep rig strung out (through power braking) while going } \\
\text { downhill } \\
\text { Apply light (5 pounds) brake pressure continuously } \\
\text { Select a gear that will permit keeping engine speed at } \\
\text { about half power }\end{array}$ \\
\hline & Passing & $\begin{array}{l}1 . \\
2 . \\
3 .\end{array}$ & $\begin{array}{l}\text { Determine if sufftcient speed and distance } \\
\text { Make smooth transition when changing lanes to avoid whipping } \\
\text { trailer } \\
\text { Judge the distance, as seen through rear-view mirror, to determine } \\
\text { when to return to driving lane }\end{array}$ \\
\hline
\end{tabular}




\begin{tabular}{|c|c|c|c|}
\hline \multicolumn{2}{|r|}{ Tasks } & \multicolumn{2}{|r|}{ Subtasks } \\
\hline III. & Surveillance and Situation Awareness & $\begin{array}{l}1 . \\
2 . \\
3 . \\
5 . \\
5 .\end{array}$ & 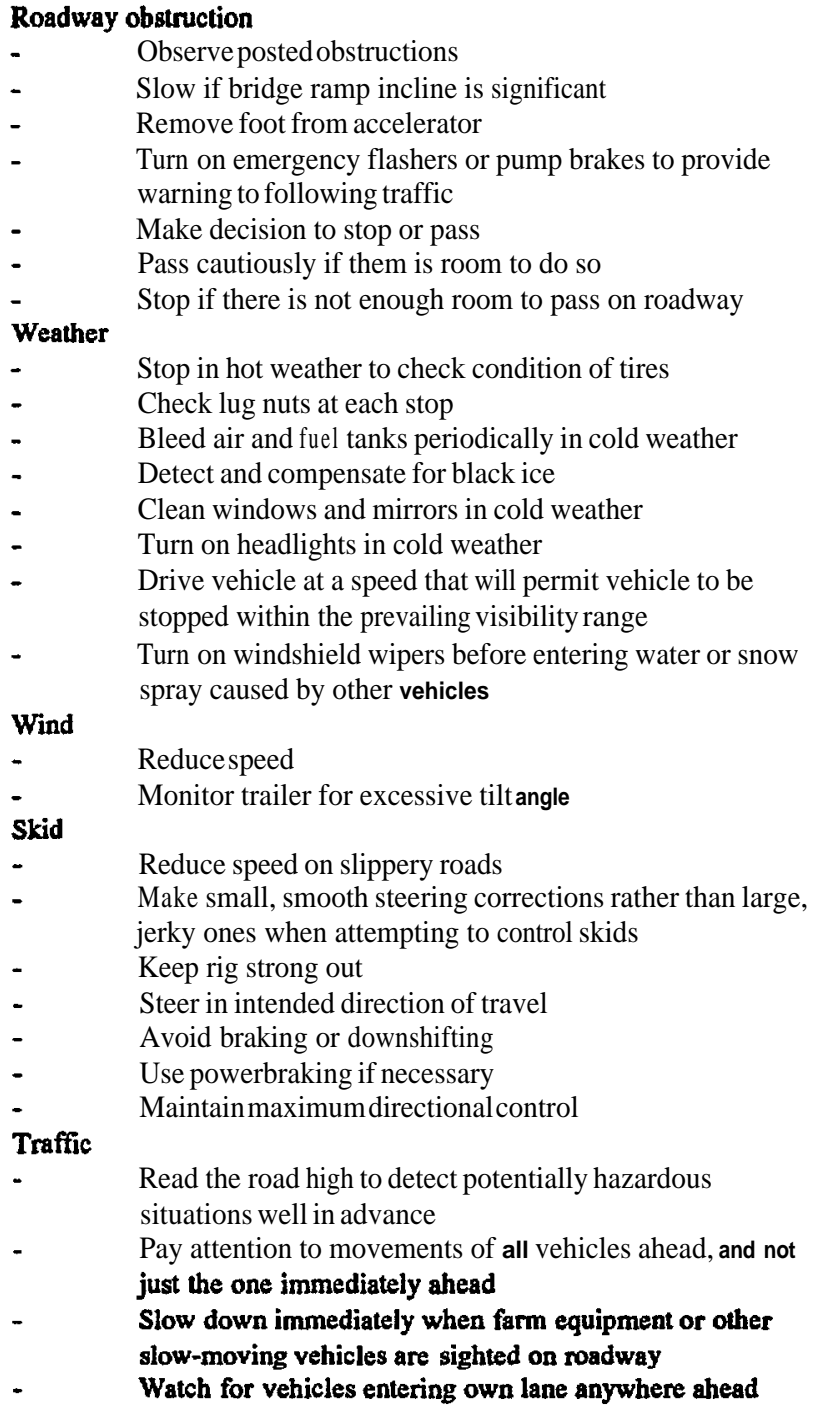 \\
\hline & Braking and Stopping & $\begin{array}{l}- \\
- \\
-\end{array}$ & $\begin{array}{l}\text { Avoid sharp braking on turns or curves } \\
\text { Apply steady pressure on foot brake when initiating stop } \\
\text { Avoid locking wheels to maintain directional control of vehicle } \\
\text { Pump brake pedals to dry wet brakes }\end{array}$ \\
\hline & On-the-road Inspections & 1. & $\begin{array}{l}\text { Route rest and refueling stops } \\
\text { - Check fifth wheel }\end{array}$ \\
\hline IV. & $\begin{array}{l}\text { Special Driving Tasks } \\
\text { A. } \quad \text { Turnabouts }\end{array}$ & $\begin{array}{l}1 . \\
2 .\end{array}$ & $\begin{array}{l}\text { Avoid making U-turns or Y-turns } \\
\text { Reverse direction by driving around block }\end{array}$ \\
\hline & Backing up & $\begin{array}{l}1 . \\
2 . \\
3 . \\
4 . \\
5 . \\
6 . \\
7 .\end{array}$ & $\begin{array}{l}\text { Avoid backing whenever possible } \\
\text { Back to the left when possible } \\
\text { Get out and make visual inspection of the area to the rear of the } \\
\text { vehicle } \\
\text { Station someone to the rear of the vehicle to act as signalman } \\
\text { Signal intention to back (accelerate engine, sound born, turn on } \\
\text { flashers) } \\
\text { Back slowly in lowest reverse gear, scanning all mirrors sequentially } \\
\text { To back a trailer, steer a heading opposite the desired direction of } \\
\text { travel until the trailer is moving in the desired direction of travel, } \\
\text { then steer a heading the same as the desired direction of travel to line } \\
\text { up the tractor. }\end{array}$ \\
\hline
\end{tabular}




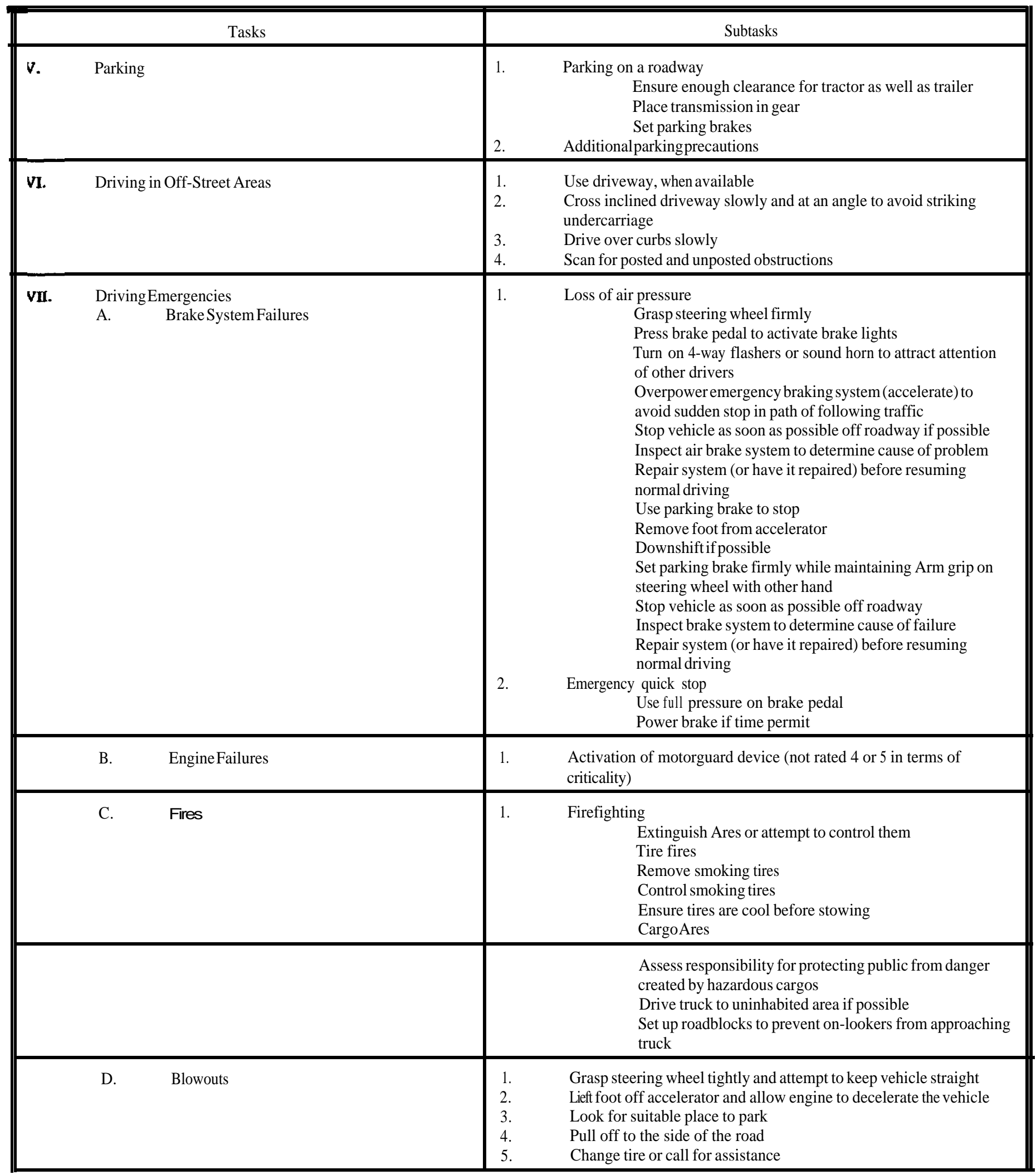




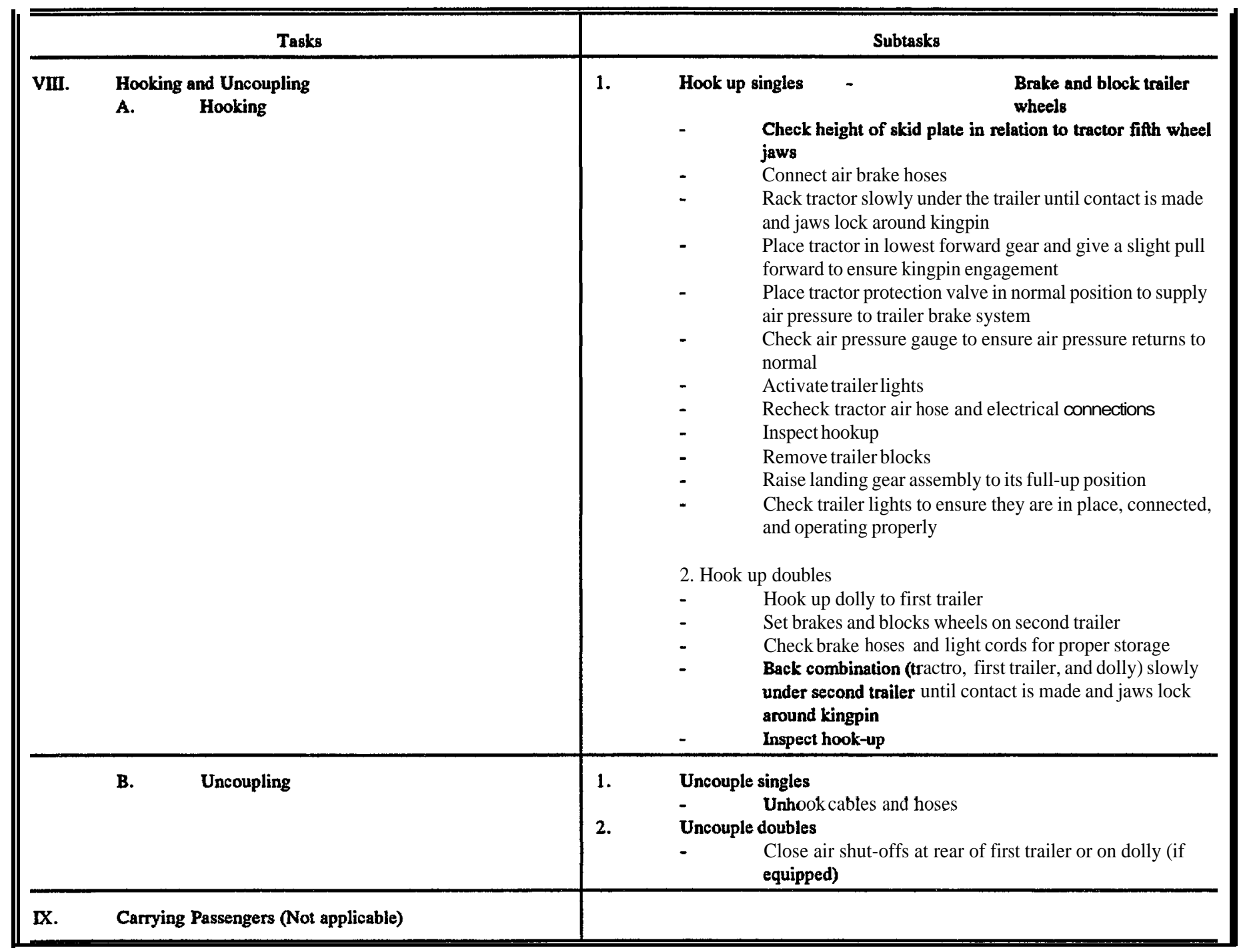


Driver Information

Needs:

Operational

Validity:

Driving

Conditions:

Criteria for

Safety Thresholds:

Usefulness

of Data:

Risk Taking

Behavior:

Reviewer:
None explicitly given.

High, given the number of driver inputs used to generate the tasks.

None specified (but see specific tasks, e.g., Weather, Wind).

None provided.

This listing provides a rich source of details about driving tasks. It contains the type of data one might expect to extract from a protocol analysis. It is anticipated to be the source of task analytic work in future phases of the NHTSA workload project.

Not discussed.

L. Tijerina 
Spolander, K. (1980). Professional Driver Training for the 1980's. Truck and Bus Driver Task Analvsis. Linkoping, Sweden: National Swedish Road and Traffic Research Institute. (In Swedish, portions translated by Battelle).

Data Type: $\quad$ Formal task aualysis with driver expert review and validation.

Task Listing: In this analysis, 211 tasks were identified and grouped into nine categories: vehicle inspection, loading, unloading, vehicle techniques, maintenance, passenger transportation, laws and rules administration, man as a driver, and other vocational matters. From these, the tasks given in Table 2.4 were identified as most relevant to the NHTSA project.

Task Structure: $\quad$ None.

Driver: $\quad$ Each task was judged on four dimensions by a total of 487 drivers, driving instructors, local councils of professional drivers, and employers. These were frequency, safety criticality, transportation efficiency, difficulty.

Timeline

Analysis:

None.

Driver Information

Needs: None.

Operational

Validity:

High, given the large number of truck drivers who reviewed and rated each task.

Driving

Conditions: None.

Criteria for

Safety Thresholds:

None provided.

Usefulness

of Data:

This data provides corroboration for safety-critical tasks involved in truck driving. This analysis and listing is particularly interesting because it provides not only relative safety-criticality information but also relative difficulty and frequency information as well. If it can be assumed that workload is higher for more difficult tasks, then these tasks merit further inquiry during subsequent phases of the current project. 


\section{TABLE 2.4 SELECTED TRUCK AND BUS DRIVER TASK ANALYSIS (TRANSLATED FROM SPOLANDER, 1980)}

NOTE: F=Frequency, S=Safety Criticality, E=Effectiveness for Transportation, and D=Difficulty. Values Presented are Integer standard scores.

*=Not as Important to American Drivers as Judged by Battelle Driver SME.

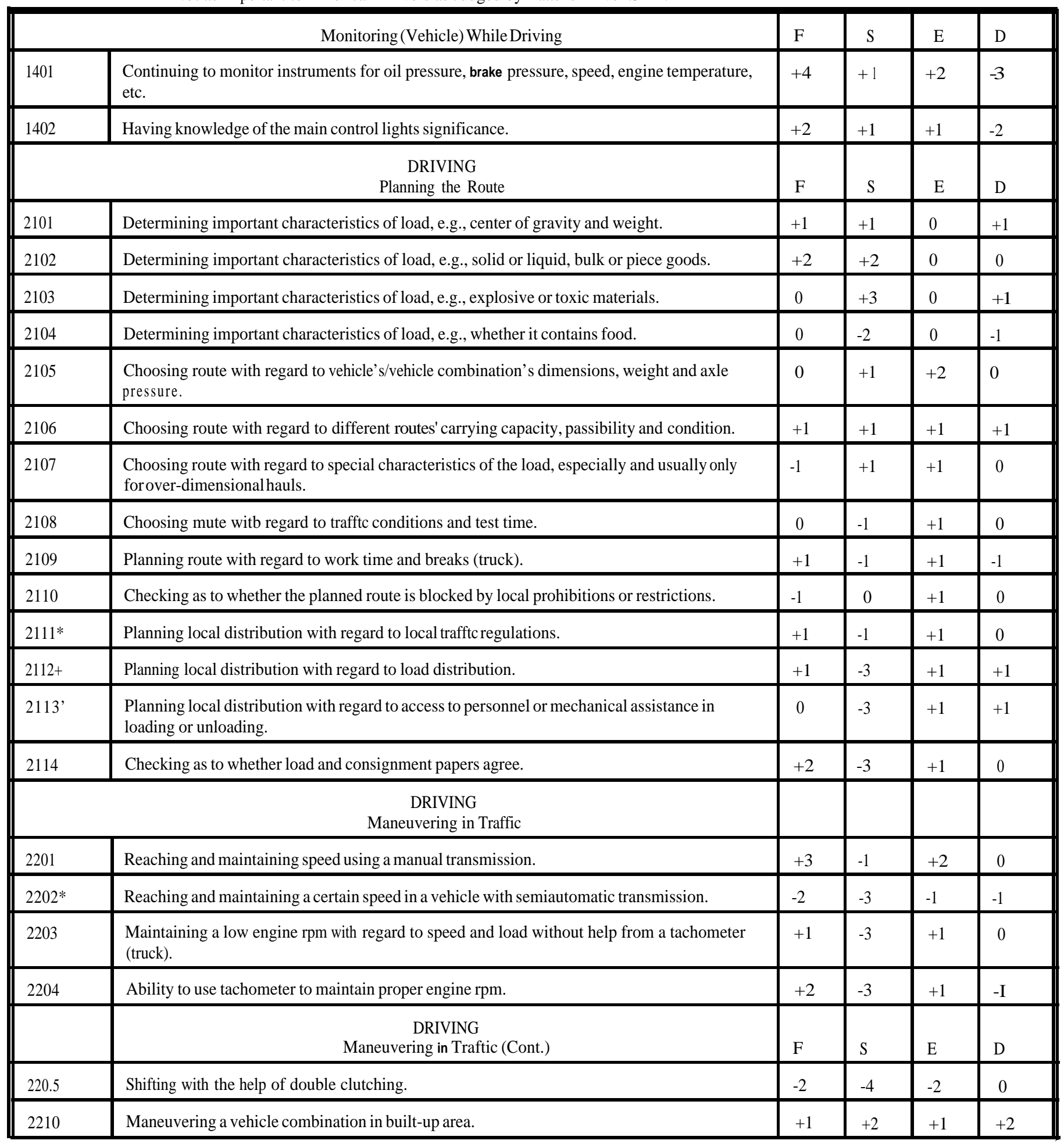




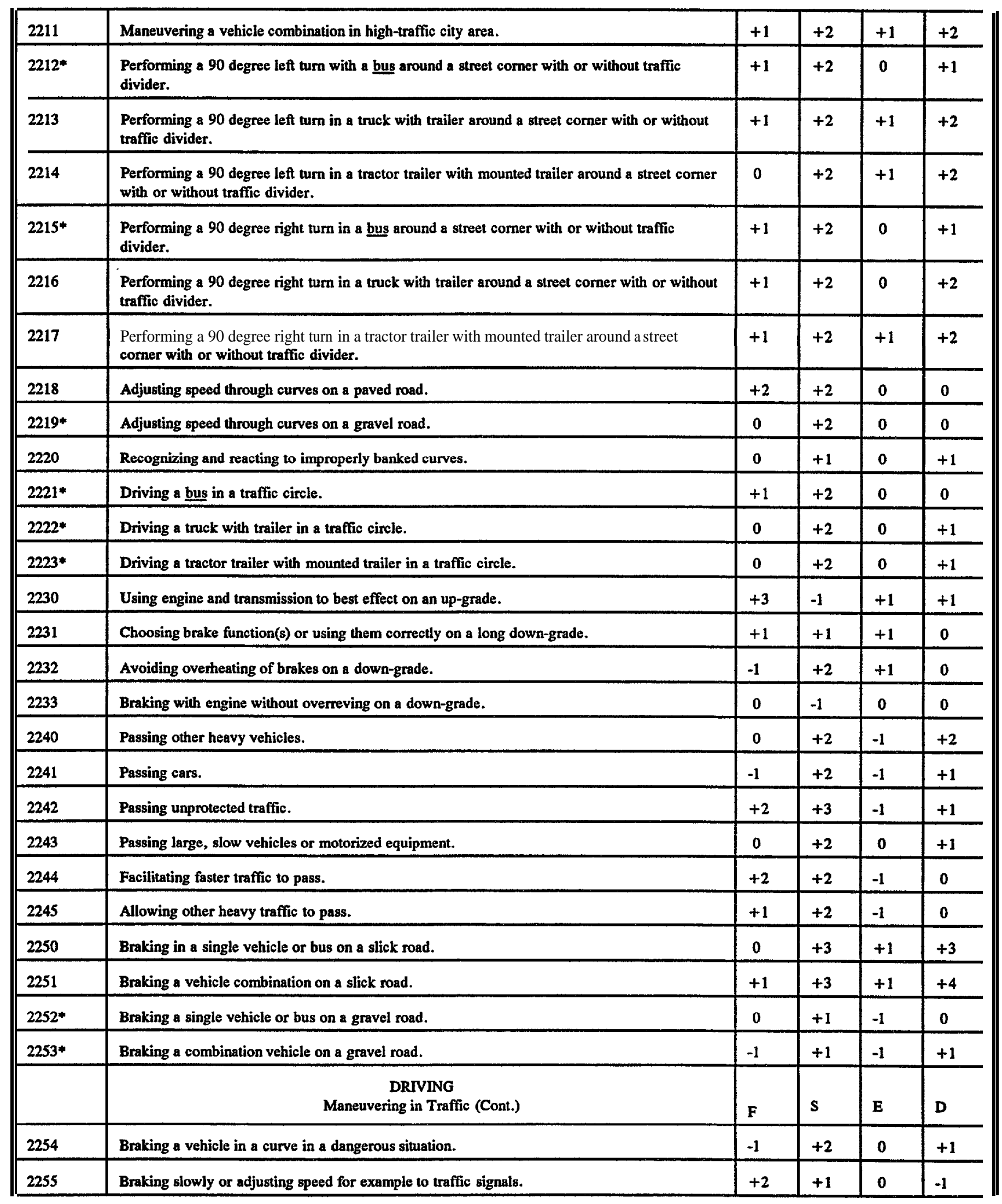




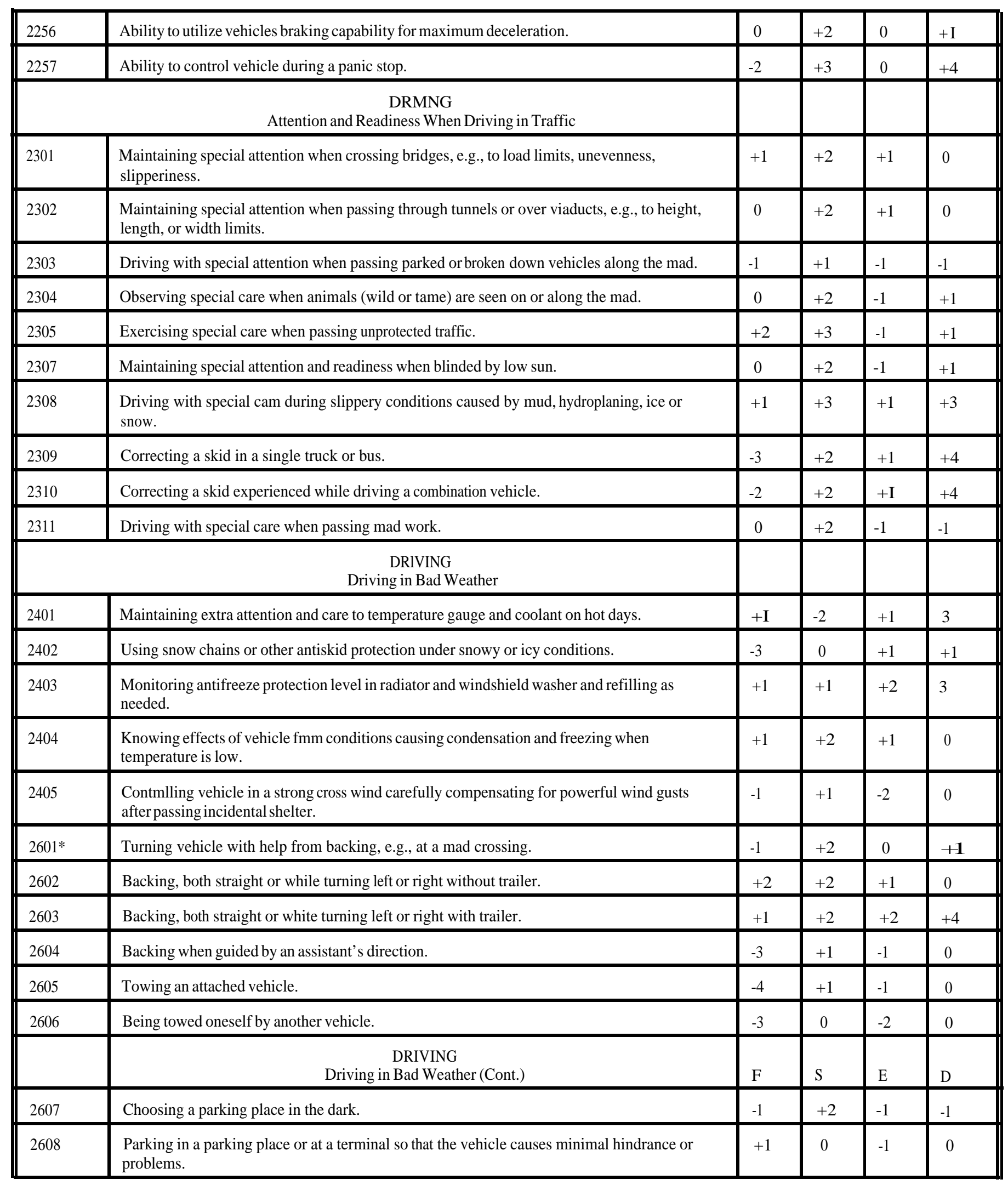




\begin{tabular}{|c|c|c|c|c|c|}
\hline 2609 & Parking on an up-grade. & -1 & +1 & -1 & -1 \\
\hline \multirow[t]{2}{*}{2610} & Parking on a down-grade. & -1 & +1 & -1 & -2 \\
\hline & $\begin{array}{c}\text { DRIVING } \\
\text { Unforeseen Occurences When Driving }\end{array}$ & & & & \\
\hline 2701 & Ability to stop when air (brake) pressure is lost or during other brake failures. & -2 & +2 & +1 & +2 \\
\hline 2702 & Stopping when indicator shows low oil pressure. & -3 & -1 & +2 & -3 \\
\hline 2703 & Stopping when engine temperature is too high. & -3 & -2 & +1 & -3 \\
\hline 2764 & Steering vehicle with a punctured front tire. & $1-4$ & +1 & -1 & +3 \\
\hline 2705 & Steering vehicle with partial or complete failure of power steering. & -3 & +2 & -1 & +1 \\
\hline 2706 & Removing air from fuel system atter fuel has run out in a diesel engine. & 3 & -3 & +1 & 0 \\
\hline 2710 & Coping witb an engine fire. & -4 & +1 & +1 & +2 \\
\hline 2711 & Coping with a vehicle body fire (bus). & -4 & +3 & +1 & +4 \\
\hline 2712 & $\begin{array}{l}\text { Coping with a fire in a load consisting of normal combustible material (wood, paper or similar } \\
\text { material). }\end{array}$ & $I^{-4}$ & +1 & +1 & +3 \\
\hline 2713 & $\begin{array}{l}\text { Taking protective measures or measures to extinguish fire in a load consisting of explosive or } \\
\text { dangerous materials. }\end{array}$ & -4 & +2 & +1 & +4 \\
\hline 2720 & Assisting injured persons in a traffic accident. & -4 & 0 & -2 & +4 \\
\hline 2721 & Warning and directing other traffic around a traffic accident. & -3 & +2 & -1 & $\mathbf{0}$ \\
\hline \multirow[t]{2}{*}{2722} & Recording names and data relating to sequence of events in a traffic accident. & -2 & -3 & 0 & -1 \\
\hline & LOADING AND UNLOADING & & & & \\
\hline 3001 & Loading and securing piece goods or palleted goods. & +2 & +2 & +2 & +2 \\
\hline 3002 & Loading and unloading bulk or mass goods. & -1 & -1 & 0 & +1 \\
\hline 3003 & Loading and securing load of lumber or timber. & 0 & +3 & +2 & +1 \\
\hline 3004 & Loading and unloading a tank truck. & 0 & +1 & +1 & 0 \\
\hline 3005 & Covering load with a tarpaulin. & -2 & -1 & 0 & -1 \\
\hline 3006 & Knowing where directions and requirements on securing load are found. & 0 & +2 & +1 & +1 \\
\hline 3007 & Securing and stowing goods according to current requirements. & +2 & +2 & +1 & +1 \\
\hline 3008 & Marking a protruding load. & -1 & +2 & -1 & -2 \\
\hline 3009 & Using vehicle mounted crane for loading and unloading. & -2 & & +1 & +1 \\
\hline 3014 & Loading with regard to requirements for legal load, axle and wheel unit weight. & +3 & +2 & +1 & +2 \\
\hline
\end{tabular}


Risk Taking

Behavior: None addressed in the task descriptions themselves.

Methodology: Formal analysis with written/interview validation by a large sample of truck drivers.

Reviewer: L. Tijerina

Professional Truck Driver Institute of America (PTDIA). (April, 1989). Criteria for voluntarv certification of tractor-trailer driver training courses and curriculum. Elk Grove, CA: Author.

Data Type: Task listing derived from Training Curriculum.

Task Listing: See Table 2.5.

Task Structure: $\quad$ None provided.

Driver: $\quad$ None mentioned. However, given PTDIA as the source, assume significant inputs from professional truck drivers.

Timeline

Analysis:

None.

Driver Information

Needs: $\quad$ None explicitly described.

Operational

Validity: High, given that this is the sanctioned truck driver training curriculum in the United states.

Driving

Conditions: None specified.

Criteria for

Safety Thresholds: None provided.

Usefulness

of Data: $\quad$ Provides additional indication of the core driving tasks which truck drivers must execute.

Risk Taking

Behavior: Not addressed.

Methodology: Not described.

Reviewer: L. Tijerina 
TABLE 2.5. PTDIA DRIVER CURRICULUM (ADAPTED FROM PTDIA, 1989).

\begin{tabular}{|c|c|}
\hline BASIC OPERATION & \begin{tabular}{ll} 
1.1 Orientation & \\
1.2 Control Systems & \\
\multicolumn{3}{l}{} & operate instruments and controls (e.g., gear shift, tach, air gauge, low air \\
warning, etc.) & Pehicle Inspection \\
- & Pm-tripinspection \\
- & Inspection while in operation \\
- & End-of-tripinspection \\
1.4 Basic Control & Starting \\
- & Stopping \\
- & Backing \\
1.5 Shifting & \\
1.6 Backing & \\
1.7 Coupling and uncoupling
\end{tabular} \\
\hline $\begin{array}{l}\text { SAFE OPERATING } \\
\text { PRACTICES }\end{array}$ & $\begin{array}{cl}\text { 2.1 Visualsearch } & \\
\text { 2.2 Communication } & \\
- & \text { signaling, } \\
- & \text { use of horn } \\
\text { - } & \text { other } \\
\text { 2.3 Speed management } & \text { maintaining speed in a variety of situations } \\
- & \text { operating on hills, } \\
- & \text { curves } \\
\text { - } & \text { other } \\
\text { 2.4 Space } & \text { management } \\
- & \text { following distances } \\
\text { - } & \text { keeping space to the sides and rear } \\
\text { - } & \text { passing } \\
\text { 2.5 Night operation } & \text { other } \\
\text { 2.6 Extreme driving conditions } \\
\text { - } & \text { cold and hot weather } \\
\text { - } & \text { stormyconditions } \\
- & \text { mountainous terrain } \\
- & \text { installing chains and towing a stuck vehicle }\end{array}$ \\
\hline $\begin{array}{l}\text { ADVANCED OPERATING } \\
\text { PRACTICES }\end{array}$ & $\begin{array}{l}\text { 3.1 Hazard perception } \\
\text { 3.2 Emergency } \\
-\quad \text { maneuvers } \\
-\quad \text { emergency braking } \\
-\quad \text { evasive actions } \\
\text { 3.3 skid control, jackknifing, and recovery }\end{array}$ \\
\hline VEHICLE MAINTENANCE & $\begin{array}{l}\text { 4.1 Vehicle Systems } \\
\text { 4.2 Preventative Maintenance and Servicing } \\
\text { 4.3 Diagnosing and Reporting Malfunctions }\end{array}$ \\
\hline NON VEHICLE ACTIVITIES & $\begin{array}{l}\text { 5.1 Handling Cargo } \\
\text { 5.2 Cargo Documentation } \\
\text { 5.3 Hours of Service Requirements } \\
\text { 5.4 Accident Procedures } \\
\text { 5.5 Personal Health and Safety } \\
\text { 5.6 Trip planning } \\
\text { 5.7 Public and employer relations }\end{array}$ \\
\hline
\end{tabular}


Friesen, Kaye and Associates (1990, November). An occupational analvsis leading to the development of a professional truck driver curriculum. Prepared for the Canadian Truck Transport Industry.

Data Type: Unknown. No details of method used to derive tasks. This is a task listing to be used to develop a truck driver training program. However, it provides details of the driver activities and skills needed to various driving tasks.

Task Listing: See Appendix 2A at end of this section. (Note: This material was received very recently and thus has not yet been reformatted).

Task Structure: None.

Driver: None specified.

Timeline

Analysis: $\quad$ None.

Driver Information

Needs:

Needs may be inferred from descriptions of individual tasks.

Operational

Validity:

High based on comparison with other listings.

Driving

Conditions: None specified.

Criteria for

Safety Thresholds: None explicitly provided.

Usefulness

of Data: $\quad$ This is the most current task analysis list found to date. It includes a relatively fine grained description of tasks under the Skill/Knowledge heading. This is expected to corroborate (and update) Moe et al., and other sources.

Risk Taking

Behavior: Not discussed.

Methodology: Methods used are not reported in the documentation we have received.

Reviewer: L. Tijerina

McKnight, A.J. and Adams, B.B. (1970). Driver education task anaivsis. Volume I: Task descriptions. Final report (Report No. DOT-HS-800-367; HumRRO-70-103). Washington, DC: National Highway Traffic Safety Administration. 
McKnight, A.J. and Adams, B.B. (1970). Driver education task analvsis. Volume II: Task analysis methods. Final reuort (Report No. DOT-HS-800368; HumRRO-IR-DI-70-I). Washington, DC: National Highway Traffic Safety Administration.

McKnight, A.J. and Hundt, A.G. (1971). Driver education task analvsis. Volume UI: Instructional obiectives. Final reuort (Report No. DOT-HS-800-369; HumRRO-71-9). Washington, DC: National Highway Traffic Safety Administration.

McKnight, A.J. and Hundt, A.G. (1971). Driver education task analysis. Volume IV: The development of instructional objectives. Final reuort (Report No. DOT-HS-800-270; HumRRO72-14). Washington, DC: National Highway Traffic Safety Administration.

Synopsis: $\quad$ The overall objective of this study was to develop "performance-oriented driver education objectives" and instruments to measure attainment of those objectives. Tire results of tbe study are presented in four volumes, as referenced above.

In Volume I, the detailed descriptions of passenger car driver tasks were presented. A total of 45 tasks [see Table 2.61, involving 1,500 driver behaviors, were identified. The list of tasks identified in this report follows later in this summary. The tasks were divided into two major categories: on-road and off-road tasks. In the on-road category, the basic control tasks and tbe general driving tasks were performed throughout all driving, while the remaining tasks in the category were situationoriented tasks. Tasks listings were detailed (i.e., subtasks were included) and other supporting information was described. Supporting information was classified into the following categories: performance information,. performance limits, criticality information, skill information (i.e., perceptual, motor or cognitive processes) and knowledge information. A safety criticality index (range \pm 20 ) was also included for each task and subtask The sample listing for "Skid Control" is provided in Table 2.7.

Volume II described the method used to identify the driver tasks and the method used to obtain the safety criticality index. The identification of driver tasks was based on an analytic procedure that involved: (1) identification of transportation system characteristics that produce situations to which a driver must respond, (2) identification of required driving behaviors, (3) organization of driving behaviors into tasks and (4) detailed analysis of the tasks. The safety criticality of tasks was developed using the judgements of experts in driver education, traffic safety, law enforcement and driver licensing. One-hundred evaluators each ranked three lists of 25 randomly selected driving behaviors from the task analysis in terms of criticality to the overall transportation system. The rankings were normalized and the mean for each task was calculated. An ANOVA indicated good inter-judge agreement.

Volume III of the study outlined the instructional objectives for a driver training program along with the evaluation instruments needed to assess driver performance and knowledge. The objectives are presented in learning units which were designed to assist driver educators to revise or develop new curricula. The evaluation 
instruments included a driving fundamentals test, driving situations test and a driving knowledge test.

Volume IV presents the methods used to develop the instructional objectives.

Note: Volumes III and IV are of little relevance to the truck driver workload project.

Method(s): Analytic

Task Listing: Primary and ancillary tasks described.

Safety

Criticality: Index based on mean normalized rank of task.

Time Line

Analysis:

None.

\section{Detailed Human}

Performance

Descriptions: Includes some description of the perceptual, cognitive and motor requirements of a task. No loading data was provided. Some quantitative data on driver performances are also provided.

Subjects:

100 subject matter experts were used to rate the safety criticality of driving tasks and subtasks. Experts consisted of driving educators, safety specialists and law enforcement personnel.

\section{Driving}

Conditions: No driving performed.

Validity

Issues:

Task analysis was developed for training of automobile drivers not for already-trained truck drivers. Task descriptions must be reviewed as must the criticality indices before this data could be used for truck driving.

Risk Taking

Behavior:

Not discussed.

Usefulness of Data:

There was a relatively high level of detail regarding the task descriptions. Primary and ancillary task listing are provided, along with some data on the requirements of the task. No time line data was presented. Although this analysis pertains to automobile driving, it could serve as a good beginning place for a truck driver task analysis if no suitable one is identified elsewhere in the literature review.

Reviewer: $\quad$ S. Kiger 
TABLE 2.6 DRIVER EDUCATION TASK ANALYSIS (SOURCE MCKNIGHT ET AL.)

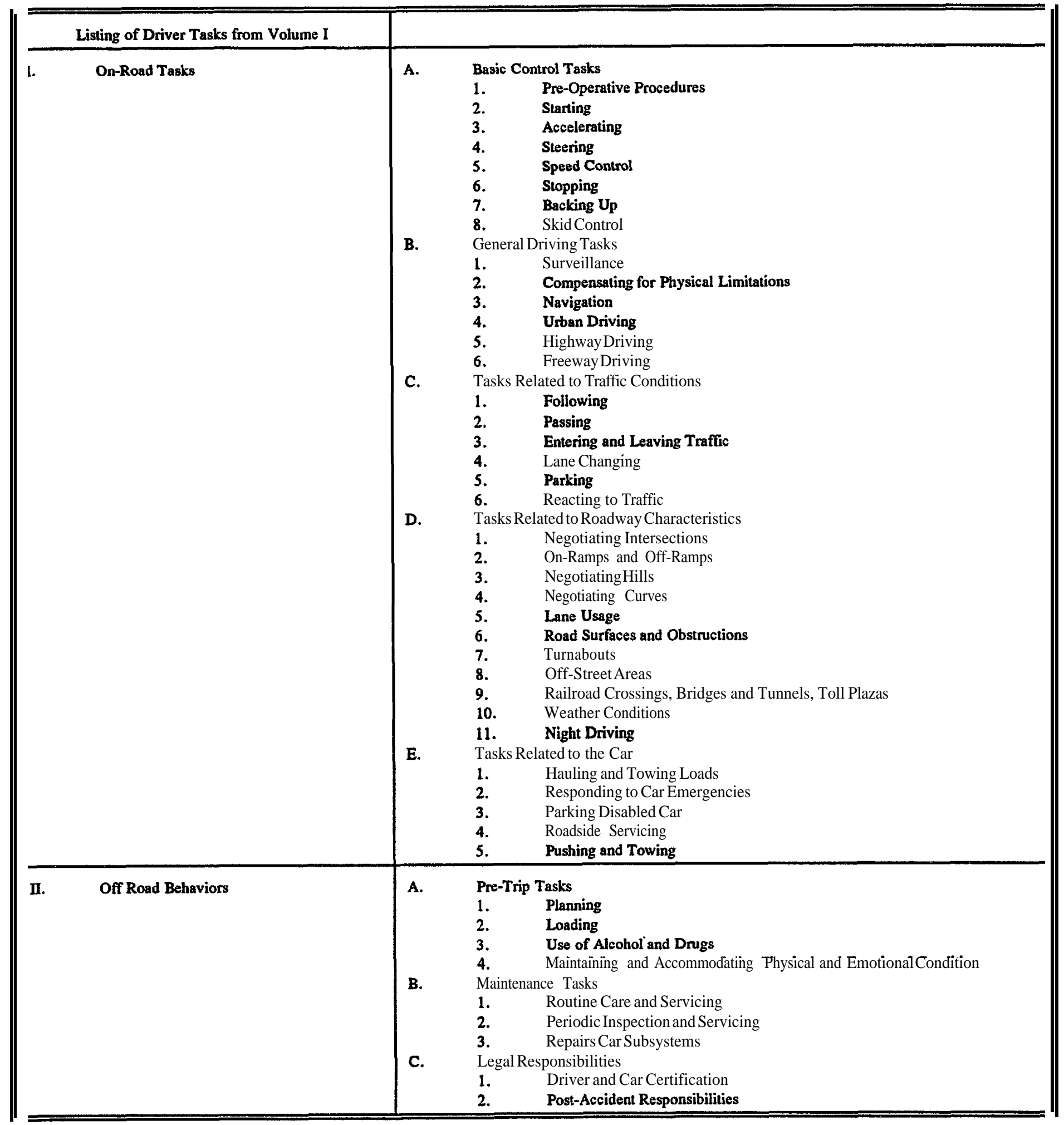




\section{TABLE 2.7 TASK LISTINGS AND ANALYSIS FOR SKID CONTROL [SOURCE MCKNIGHT ET AL.)}

Task 18: Skid Control

18.1

18.11

18.12

18.13

18.22

$18 \cdot 3$

$18-31$

183

18.4

18.4

1842

$18-9$

18.5

18.51

18.52

$18-521$
ANTICIPATES SKID PRODUCING SITUATION SUCH AS:

Qrvesor turns at excessive speed

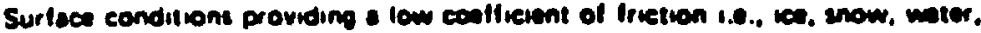

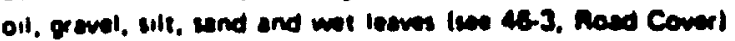

E xcessive rate of deceleration

TAKES PREVENTIVE MEASURES TO AVOID SKIDS

Enters curves or turns at moderate speeds

When driving on slippery surfaces, avoids abrupt changes in car velocity $\infty$ direction of movement

Perceives diccropancy between cars heading and path ongle Idirection car is actually movinel *

Percerves leck of decoleration upon braking

ATTEMPTS TO ARREST SKID .

Gradually releases pressure on accelerator pedal

$6 \times \times \times \times$

Keop foot oll brake pods -

$\mathbf{x} \times \mathbf{x} \times \mathbf{x}$

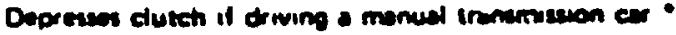

$\mathbf{x} \times \mathbf{x}$

COUNTERSTEERS CAR TO CORRECT FOR THE SKID •

Immediately turns front wheels in the direction of desied car leatg

16

$\mathbf{x} \times \times \times$

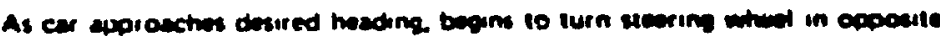

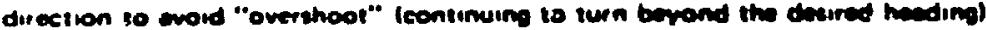

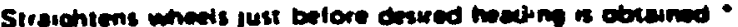

$9 \times \times \times \times$

- $\times \times \times \times$
Criticality

$\times \times \times \times x$ 
TABLE 2.7 (Continued)

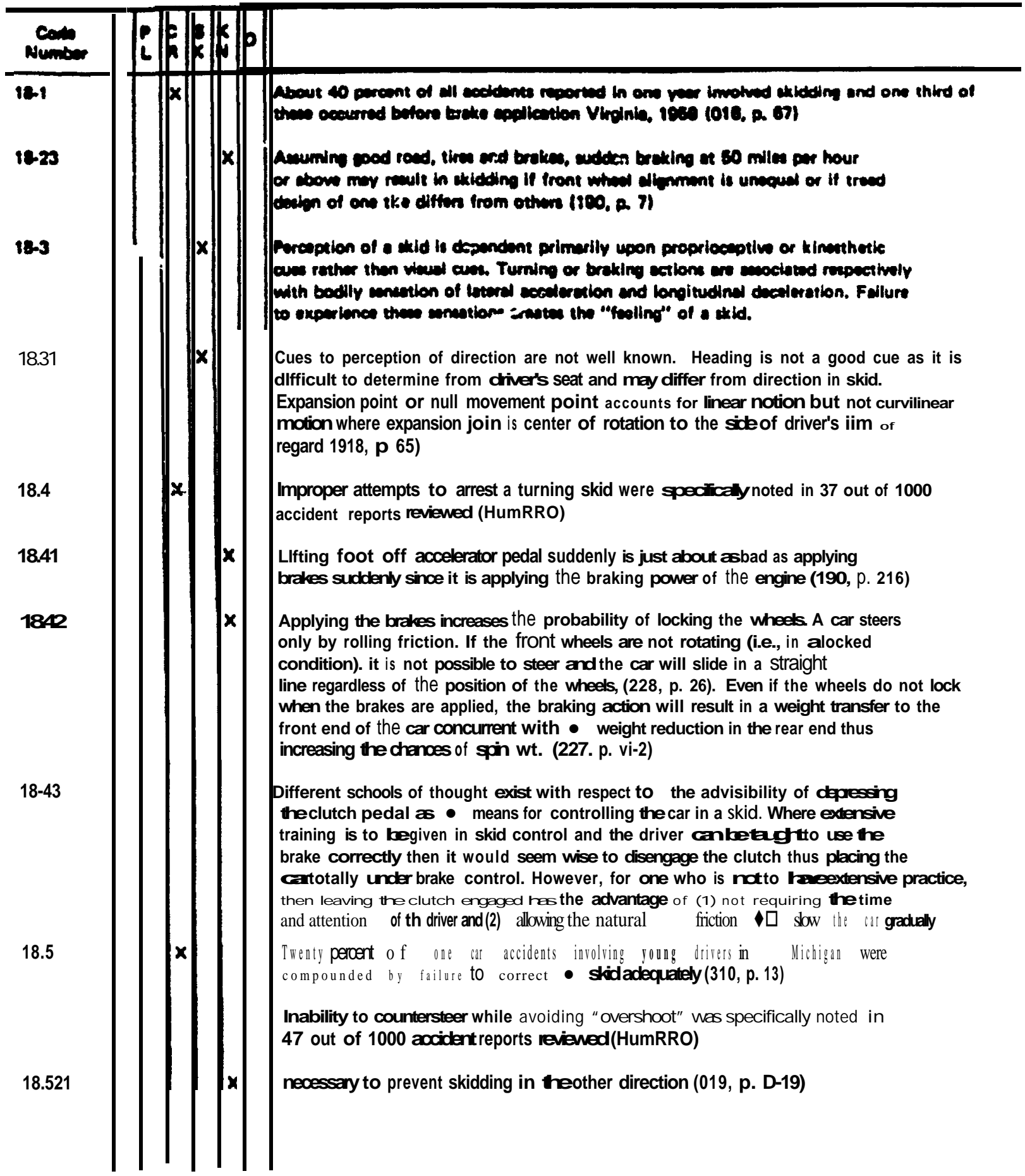


Eberhard, J.W. (1969). Driver Information Requirements and Acceptance Criteria. Highway

Research Record No. 25. Washington, DC: Highway Research Board. pp. 1930.

Synopsis: $\quad$ To support development of in-car displays for the Electronic Route Guidance System (ERGS), an analysis of driver tasks required to negotiate a generic intersection was performed. The analysis was performed from the perspective of an unfamiliar driver using an electronic way finding system like ERGS. Twenty-two tasks were identified (See Table 2.8). Worst case execution times were also presented for two maneuvers: a lane change (See Table 2.8) and a speed change (See Table 2.8).

In a survey of 561 licensed drivers, user acceptance of the ERGS concept, display design features, desired roadway types for implementation and willingness to buy were investigated.

Data Type: Formal analysis and user survey.

Task Listing: Primary and ancillary driving tasks: intersection negotiation assuming use of an electronic in-vehicle display. No detailed descriptions of tasks.

Task Structure:

None.

Driver: $\quad$ Not specified but navigation system under study intended for unfamiliar drivers (first time in an intersection) and aged drivers.

Safety

Criticality: Critical tasks (changing lanes and changing speed) identified for negotiating an intersection, but basis for this determination was not explained.

Time Line Analysis:

Worst case execution times for lane change and speed change maneuvers were presented. Appendices C and D from Eberhard, J. W. Driver Information Requirements, Display Concepts and Acceptance Factors for an Electronic Route Guidance System. Serendipity, Inc., Report No. TR 301-69-12, Contract No. FH 116805 for Bureau of Public Roads, Feb., 1969 were cited for this data but were unavailable for this review.

\section{Detailed Human}

Performance

Descriptions: None. No performance or safety criteria.

Subjects: $\quad$ For task analysis: None.

For user survey: Licensed drivers visiting the History and Technology Museum of the Smithsonian Institution. 
Driving

Conditions: No driving performed.

Operational

Validity: Not applicable.

Risk Taking

Behavior: Not discussed.

Usefulness

of Data:

Limited to enumeration of tasks. No information on cognitive, perceptual or motor requirements of the tasks. No information on task difficulty. No detailed task descriptions.

Reviewer: $\quad$ S. Kiger and B. Kantowitz (combined reviews) 


\section{TABLE 2.8. DRIVER TASKS FOR INTERSECTION NEGOTIATION, AND LEAD TIME DATA FOR LANE CHANGE AND SPEED CHANGE MANEUVERS \\ (SOURCE: EBERHARD, 1969).}

a)

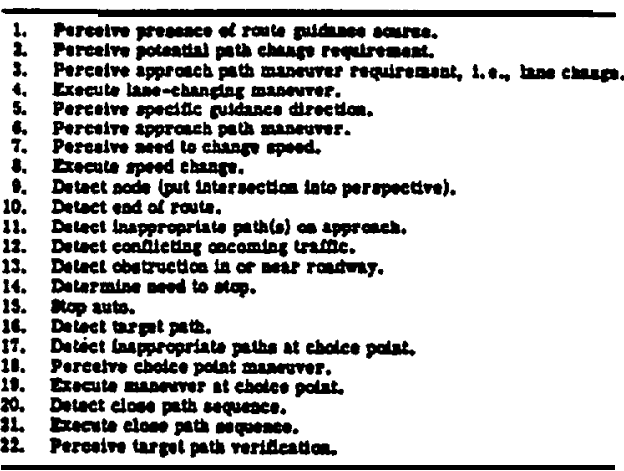

b)

\begin{tabular}{|c|c|c|c|}
\hline ramesen & inimis & Arenone & 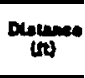 \\
\hline 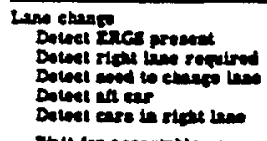 & $\begin{array}{l}2: 1 \\
1: \\
1: 5 \\
\text { i. }\end{array}$ & 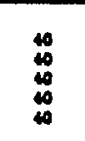 & 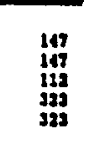 \\
\hline 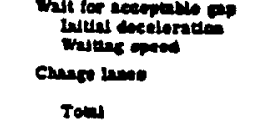 & $\begin{array}{c}1.4 \\
25.4 \\
4.4\end{array}$ & 焉 & $\frac{12,14}{1212}$ \\
\hline
\end{tabular}

c)

\begin{tabular}{|c|c|c|c|}
\hline תmatem & $i=0$ & Aperpon & Non \\
\hline 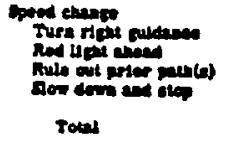 & $\begin{array}{l}\mathbf{3} \\
\mathbf{3} \\
\mathbf{p}\end{array}$ & 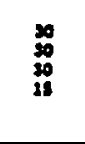 & 崫 \\
\hline
\end{tabular}




\section{$\underline{\text { Summarv on Task Descriptions and Criticality Assessment }}$}

The references reviewed here provide a significant database of driving tasks with which to guide further efforts on this project. The work by Moe, et al. (1973) and M\&night and his collegues, in particular, offers a significant degree of detail with regard to task and subtask descriptions. In general, however, ancillary tasks (e.g., HVAC adjustments, CB operations, or other in-cab tasks) are not described and neither are additional informal driver behaviors (such as pouring coffee, fumbling with snacks, or lighting a cigarette) which may affect driver workload.

With regard to task structure, the task listings suggest the sequential nature of processes. But traditional task analyses cannot be interpreted in this light. The task listings provided by these sources by and large are hierarchical decompositions of the driving tasks garnered from a variety of sources e.g., observations, driver SME inputs, armchair analysis, theoretical considerations, etc. The degree to which task structure must be described for our purposes is not clear but, at a minimum, concurrent task execution seems essential to our understanding and these task descriptions do not provide this.

Driver descriptions are usually absent or vague. There are, on the other hand, a number of references which address variations in driver performance and workload as a function of various driver variables, namely experience, age, familiarity with the route, and cognitive style. These references will be discussed as part of Battelle's Task 4 Interim Report.

Unfortunately, no timeline analyses were found (though Eberhard does provide some task duration data). Perhaps this is because of the dynamic nature of driving. Rabideau and Young, for instance, preferred an event-based mission approach rather than a time- or distance-based mission approach because of highly variable driving conditions. Thus, timelines might not be appropriate. Frequency of occurrence is important, however, for understanding the nature of background concurrent tasks and for prioritizing tasks to be included in as standard driving tasks in a workload assessment. Therefore, frequency of occurrence, which was originally thought to be available from a timeline analysis, is still a task analysis data need. The work of Spolander contributes to this to some extent.

Safety criticality was addressed in several sources using rating and ranking procedures. These ratings will be used to select tasks which are analyzed further in Tasks 2, 3 and beyond. Given that somewhat different definitions of safety criticality were used, there is still a need to verify these assessments, in our estimation. The project staff driver SME consultant provides a useful verifier, but confirmation from a sample of drivers is desirable to avoid any possibility of bias. A common definition of safety criticality is also needed for this purpose.

Driver information needs were addressed indirectly in the task descriptions given in the various reports. However, the only formal presentation of driver information needs is that provided by Rabideau and Young (1973) in Table 2.1. Upon looking at this level of task description, it is unclear how much more is needed. It seems at this point that defining what the driver's eyes, ears, hands, feet, and mind are doing at any given time while at the wheel is more appropriate to our purposes. Therefore, it is recommended that Task 3 data collection NOT explicitly be directed toward filling out such tables for each and every task and subtask to be analyzed. A more reasonable and cost-effective approach will be to augment Table 2.1 with any variations that come up in the course of other, more appropriate, task analysis data collection. 
In general, the data presented in this section is devoid of descriptions indexed by or conditional on driving condition. Furthermore, some driving conditions of interest to us are missing (e.g., driving in fog). Given that driver workload is determined, at least to some extent, by driving conditions, it seems important to understand this factor in advance.

Our conclusion is that the task descriptions contained in these references are of value and provide significant advantage for this project. These task descriptions provide us with guidance on those tasks which we wish to understand further from a workload standpoint. They do, however, need to be verified for completeness and correctness in today's driving environment. They need to be augmented to some extent with additional tasks which may affect workload and safety. The concept of safety criticality needs to be standardized and the criticality assessments provided in this literature need to be verified. Task 3 data collection will be directed toward these ends. 


\section{APPENDIX 2A. PROFESSIONAL TRUCK DRIVER TASKS ANLYSIS DATA [SOURCE: FRIESEN, KAYE, AND ASSOCIATES, 1990].}

Performance Objective 12: $\quad$ Drive Defensively On Public Roads (Urban/Highway/Expressway) OBJECTIVE:

The student will demonstrate the defensive driving skills required for urban, highway, and expressway driving.

\section{CRITERION TEST:}

The student will demonstrate defensive driving techniques in a variety of road and traffic conditions under supervision.

\section{STANDARD:}

The student will drive the tractor/trailer under various traffic and road conditions under the supervision of the instructor to the standards prescribed in the instructor's checklist.

\section{CONCLUSION:}

Close supervision is required during the driving exercises.

\section{Sub-tasks}

12.1 Apply search techniques

\section{Enabling Objectives}

ability to scan to the center, sides and rear using prescribed techniques and patterns

knowledge of the relationship of search techniques to safe driving under various road, traffic and load conditions

knowledge of overhead clearance requirements

ability to judge overhead clearance requirements
Skill/Knowledge

Execute Search Techniques

- use distance scanning techniques look way ahead (10-15 seconds) steer or aim at imaginary target in the center of your path of travel scan to sides as well as center of road vary eye lead-time depending on situation (city, highway, traffic, available sight distance)

- $\quad$ look "all around" (keep eyes moving both near and far)

scan to the sides periodically scan sides when driving use left-right-left technique at intersections, crosswalks, school zones, and in other critical situations

- consider blind spots when scanning

check to the rear monitor load and cargo securement monitor for tire fires monitor adjacent and following vehicles (check minute) 


\section{Sub-tasks}

1z.2 Manage Speed

\section{Enabling Objectives}

knowledge of the relationship of speed to safe driving, fuel economy and equipment wear under various road, traffic and load conditions

knowledge of the relationship of speed to stopping distance under various road, traffic and load conditions

ability to assess driving conditions and adjust speed
Skill/Knowledge

Manage Speed

- be aware of conditions affecting safe speed

posted speed limits

traction

- visibility

- $\quad$ traffic conditions

- $\quad$ road surface characteristics

- load characteristics

- $\quad$ steering characteristics

Manage Stopping Distance

consider braking distance factors vehicle speed

- vehicle weight

- $\quad$ condition of braking components condition of road surface

consider driver response time hazard identification physical reaction time

Maintain Safe Following Distance (i.e. avoid tailgating)
- $\quad$ use timed interval principles
normal conditions equal 1 second for every 10 feet of vehicle length add extra time for hazardous conditions (I second for night, 1 or more for weather and road characteristics and 1 more for motorcycles)

Negotiate Curves

- $\quad$ reduce speed prior to curve

- downshift if necessary

- $\quad$ judge radius of curve

- $\quad$ use proper following distance

- $\quad$ keep trailer fully in lane on curve

- $\quad$ maintain power while going through curve

- $\quad$ frequently check blind side mirror

- on right turns move towards the center line on left turns move towards the shoulder 
Sub-tasks

12.3 Manage space
Enabling Objectives

knowledge of the relationship of tractor/trailer positioning relative to various road, traffic and load conditions

knowledge of the relationship of tractor/trailer positioning relative to stopping distance under various road, traffic and load conditions

ability to assess driving conditions and adjust space accordingly
Skill/Knowledge

Manage Space To The Rear

- $\quad$ avoid sudden stops or other moves

- maintain safe following distance

- $\quad$ reduce speed, if necessary, when others want to pass

Manage Space To The Sides

- $\quad$ position vehicle properly remember width of vehicle and lane width keep vehicle centered in your lane

- $\quad$ position vehicIe to account for trailer tracking when taking curves

- $\quad$ avoid cutting across lanes to straighten out a curve

Manage Space Above determine trailer height when empty (trailer, stack and fairing heights vary)

- $\quad$ ensure adequate clearance at:

- bridges and underpasses low wires tree limbs building structures overhead signs

- clearance heights indicated to be less than 6 inches above the trailer height should be checked be aware that clearances can change (i.e. under bridge when loaded but not when unloaded; in warehouse when loaded but not out) 


\section{Subtaks}

12.4 Drive defensively
Enabling Objectives

knowledge of provincial traffic codes

ability to consistently obey Provincial Highway Traffic Codes

knowledge of sound communication, speed, space and search techniques for driving under various road, traffic and load conditions

ability to consistently apply sound communication techniques while driving

\section{Skill/Knowledge}

Execute Left Turns

- turn signal on well in advance of turn

- $\quad$ get in proper lane well in advance of turn

- $\quad$ try to make eye contact with drivers/pedestrians

- $\quad$ wait to make turn with front wheels pointed straight ahead

- don't cut comer too short

- don't cut comer too tide

- don't shift during turn

- control speed

- $\quad$ keep both hands on wheel

- enter proper lane on cross street

- $\quad$ cancel turn signal promptly

- $\quad$ check mirrors during and after turn

- move towards the shoulder

Execute Right Tums

- tum signal on well in advance of turn

- $\quad$ get in right lane well in advance of turn

- $\quad$ select correct gear

- $\quad$ try to make eye contact with drivers/pedestrians

- determine, if necessary, where you will borrow space for your turn as circumstances and/or Provincial Laws allow

- $\quad$ keep traffic from passing on right side

- don't force way into intersection

- don't cut comer too short

- don't cut comer too wide

- $\quad$ avoid gear change during turn

- control speed

- $\quad$ keep both hands on wheel

- $\quad$ enter right lane on cross street

- cancel turn signal promptly

- check mirrors during and after turn

- move towards the center line 
Sub-tasks

\subsection{Drive defensively}

Enabling Objectives

knowledge of
provincial traffic
codes

ability to consistently obey Provincial Highway Traffic Codes

knowledge of sound communication, speed, space and search techniques for driving under various road, traffic and load conditions

ability to consistently apply sound communication techniques while driving
Skill/knowledge

Cross Through Intersections

- check mirrors

- $\quad$ cover the brake

- gear down for intersection

- $\quad$ reduce speed and make eye contact with stopped cross traffic

- look Ieft/right/left

- $\quad$ yield to pedestrians, other vehicles if necessary

- $\quad$ avoid making lane change in intersection avoid gear shifts in intersection

Come To Stop At Intersections

- $\quad$ check mirrors for following traffic

m. signal stop in advance by tapping brake

- $\quad$ use brake before using clutch

- use engine brake, exhaust brake or retarder when permitted

- select proper gears

- $\quad$ do not coast to a stop

e stop short of line or in sight of lead vehicle rear wheels

- do not make sudden rough stop

- do not allow vehicle to move while waiting

- $\quad$ select appropriate gear for takeoff

- $\quad$ keep clutch depressed while stopped monitor traffic all around while stopped

Starting From Intersection look left/right/left on starting on one-way street look right/left/right

- do not roll back on start

- make smooth, even get-away

- $\quad$ check mirrors 
Subtasks

\section{r2.4 $\begin{aligned} & \text { Drive } \\ & \text { defensively }\end{aligned}$}

Enabling Objectives

knowledge of provincial traffic codes

ability to consistently obey Provincial Highway Traffic Codes

knowledge of sound communication, speed, space and search techniques for driving under various road, traffic and load conditions

ability to consistently apply sound communication techniques while driving
Skill/knowledge

Cross Railway Crossings

- $\quad$ stop, look and listen at unmarked crossings

- $\quad$ look and listen for warning devices

- $\quad$ reduce speed

- downshift

- $\quad$ look both ways

- open window to listen if necessary

- $\quad$ check for second track hazard

- do not shift gears on tracks

- $\quad$ avoid brake application while on tracks

- when stopped for train neutralize transmission and apply parking brake

- $\quad$ stop if transporting dangerous goods Change Lanes

- $\quad$ check side mirror in direction of intended lane change

- $\quad$ assess gap to confirm that it is large enough to allow tractor-trailer to make lane change

- $\quad$ activate signal well in advance

- recheck mirror

- do shoulder check to cover blind spot

- $\quad$ initiate partial lane change to straddle lane divider

- maintain partial lane change position momentarily

- $\quad$ recheck mirrors

- $\quad$ complete lane change steadily and quickly

- $\quad$ cancel signal

Pass On Left (multi-lane)

- avoid passing on right whenever possible

- $\quad$ pull out (execute lane change procedure)

- $\quad$ complete pass as quickly as possible do not linger in other driver's blind spot watch left front wheel of vehicle being passed 
Sub-tasks

\subsection{Drive defensively}

Enabling Objectives

knowledge of provincial traffic codes

ability to consistently obey Provincial Highway Traffic Codes

knowledge of sound communication, speed, space and search techniques for driving under various road, traffic and load conditions

ability to consistently apply sound communication techniques while driving
Skill/knowledge

check passing side mirror to assess gap activate signal well before pulling back into lane

return steadily but quickly to the original lane

- cancel signal

Merging

- $\quad$ check main road for suitable gap activate signals align the rig on the acceleration lane check plane mirror to observe the selected gap

- $\quad$ check convex mirror for vehicles in far lanes trying to move into the gap

- $\quad$ check convex mirror for vehicles on the ramp behind the trailer pulling out to merge into the same gap

- $\quad$ adjust speed to accommodate merge point

- $\quad$ enter gap steadily and quickly

Exiting cancel signal

- $\quad$ know your exit numbers (names> so that you can plan well ahead

- $\quad$ move to correct lane well beforehand

- $\quad$ signal early

- maintain speed

- $\quad$ enter exit lane as early as possible

- $\quad$ reduce speed before reaching exit ramp curve

- $\quad$ avoid braking on the travelled portion of the main highway or in the curve

- $\quad$ use merging procedure to enter new highway or secondary road 
Sub-tasks

12.4 Drive defensively
Enabling Objectives

knowledge of

provincial traffic

codes

ability to consistently

obey Provincial

Highway Traffic

Codes

knowledge of sound

communication, speed, space and search techniques for driving under various road, traffic and load conditions

ability to consistently apply sound communication techniques while driving
Skill/Knowledge

Drive Downgrade (mountains) check brakes before descent compressor maintaining full reservoir pressure slack adjustment correct no audible air leaks glad hands and air lines secure

- $\quad$ pay attention to signs indicating angle and length of grade select proper gear before descent do not attempt down shifts on very steep slopes

- allow drive train to assist in controlling downhill speed

- $\quad$ use engine brake or exhaust brake when necessary

- apply brakes intermittently (no more than 10 p.s.i.)

- do not fan brakes

- do not shift into neutral and coast

- $\quad$ use escape ramps to avoid runaways

Drive Upgrade

- $\quad$ pay attention to signs indicating angle and length of grade

- $\quad$ check brakes at bottom of upgrade

- $\quad$ build momentum on approach

- $\quad$ select proper gear before ascent

- $\quad$ keep engine revs high enough to anticipate gear changes

- $\quad$ position vehicle in far right hand lane or in truck lane

- $\quad$ stay in lane, do not pass unless absolutely necessary

- $\quad$ use four-way flashers before speed drops below traffic speed monitor coolant and oil temperature gauges for signs of overheating 
Sub-tasks

12.4 Drive defensively
Enabling Objectives

knowledge of

provincial traffic

codes

ability to consistently

obey Provincial

Highway Traffic

Codes

knowledge of sound communication, speed, space and search techniques for driving under various road, traffic and load conditions

ability to consistently apply sound communication techniques while driving
Skill/Knowledge

Drive At Night

- $\quad$ slow down to compensate for reduced vision

- $\quad$ do not drive when fatigued adjust to low or varied roadway illumination

- do not overdrive headlights

- avoid blinding others

- $\quad$ avoid glare from oncoming vehicles

- $\quad$ keep cab lighting to a minimum

- communicate earlier (signals, horn, stops)

- $\quad$ increase following distance

Drive In Winter Conditions

- $\quad$ implement cold weather starting procedures

- $\quad$ execute proper operation on slippery surfaces

- $\quad$ start gently

make periodic brake applications (when safe) to check traction

be aware of black ice

check mirrors

adjust turning and braking to road conditions check trailer when braking to detect possible jackknife adjust speed to road conditions and visibility adjust space to road conditions and visibility

- $\quad$ install chains where required by regulation

suspend driving when conditions get very bad

avoid wet brakes. keep light application through flooded areas dry out brakes with steady gentle application

- $\quad$ suspend driving whenever conditions are personally uncomfortable (fatigue, poor visibility, stress, preoccupation, etc.) 
Performance Objective 13: Emergency Maneuvers

OBJECTIVE:

The student will be able to explain emergency braking and steering techniques.

\section{CRITERION TEST:}

The student will take a written test on emergency braking and steering techniques and a simulation test using models.

STANDARD:

The student will achieve an $80 \%$ standard on the written and simulation test.

\section{CONCLUSION:}

Written and simulation testing will be required.

sub-tasks

13.1 Demonstrate specified emergency situations using models

13.2 Explain emergency braking and steering techniques

\section{Enabling Objectives Skill/Knowledge}

knowledge of the handling characteristics of tractors and tractor/trailer combinations

knowledge of the consequences of loss of vehicle control

ability to utilize models to demonstrate consequence of loss of control

knowledge of braking and steering techniques in emergency situations
Evasive Steering

- $\quad$ brake to lower speed before reaching obstacle if there is sufficient distance

- do not brake while turning

- $\quad$ start evasive steering as soon as emergency is realized

- turn only enough to clear the obstacle

- $\quad$ use the hand-over-hand steering technique

- counter-steer as soon as front of trailer clears obstacle

- $\quad$ evade to right if vehicle is oncoming 
Sub-tasks

13.2

Explain emergency braking and steering techniques
Enabling Objectives

knowledge of braking and steering techniques in emergency situations
Skill/Knowledge

- $\quad$ if obstacle ahead is in your lane move left into oncoming lane if clear otherwise evade to shoulder

- $\quad$ if object is in middle lane of multilane road use whatever lane is clear otherwise keep straight

- $\quad$ for merging vehicles first blast horn then swerve away if necessary

- $\quad$ if swerving away puts truck in path of an oncoming vehicle then sideswipe rather than collide head on

Execute Emergency Braking

- $\quad$ use controlled braking (i.e. apply brakes just short of "wheel lockup" and maintain steady pressure on brakes)

- $\quad$ use cadence braking apply brake fully release brake partially when wheels lock when wheels begin to roll ( $\mathrm{I} / 2$ to 1 second > reapply brake

Execute Off-Road Recovery

- $\quad$ brake before turning to educe speed as much as possible

- $\quad$ avoid braking while turning

- $\quad$ keep one set of wheels on pavement if possible

- maintain as straight a course as possible

- $\quad$ if roadside is clear stay on roadside until vehicle comes to a complete stop

- $\quad$ if roadside blocked slow as much as possible and turn wheel carefully toward roadway

- $\quad$ as soon as front wheel rides up on surface turn quickly in the direction of the roadway

Recover From Brake Failure

- $\quad$ if surface level then downshift until able to use spring loaded parking brake

- $\quad$ look for escape paths (side roads, open fields, upgrades, vehicle escape ramps)

- $\quad$ create drag (scrub tires against curb, drive into heavy brush, small bushes) 
sub-tasks

$133 \begin{array}{ll}\text { Explain } & \text { knowledge of braking } \\ \text { emergency } & \text { and steering } \\ \text { braking and } & \text { techniques in } \\ \text { steering } & \text { emergency situations } \\ \text { techniques } & \end{array}$

Skill/Knowledge

Recover from Blowouts

- when a front tire blows:

grasp wheel tightly in reaction to sound or front end drop

- $\quad$ avoid braking maintain speed until vehicle is stabilized allow vehicle to slow gradually pull offroad and brake gently to a stop

- when a rear tire blows: avoid braking allow vehicle to slow and then apply brakes gently pull offroad and brake gently to a stop

Recover From Tractor/Trailer Skids

- $\quad$ remove foot from brake immediately

- $\quad$ ease off accelerator

- disengage the clutch

- use corrective steering techniques (i.e., steer in the direction of skid)

- $\quad$ use countersteering technique to avoid overshoot

- $\quad$ use countersteering technique to avoid fishtailing 


\subsection{DRIVER WORKLOAD INDICATORS AND MODELS}

\section{Introduction}

The review of workload measure-s and related data is scheduled for Task 4 of this project. However, a preliminary look at driver workload measures and models is provided here. This section is formatted in the form of outlined annotations which cover the criteria of Section 1.0. The materials here are not exhaustive of the literature in driver workload assessment and models but rather provide a range of approaches. These approaches and illustrative studies in which they have been applied will be critically reviewed in Task 6.

\section{Burger. W. J., Smith, R. J., \& Ziedman (February, 1989). Supplemental electronic in-cab} truck displavs: An inventorv of devices and approaches to their evaluation (Report No. DOT HS 807 411). Santa Monica, CA: Vector Enterprises, Inc.

Synopsis:

This source contains descriptive material and a review of various classes of incab devices which are currently available for use in heavy vehicles. These were categorized as single/integrated displays, information systems, navigation systems, positioning systems, communication systems, and vehicle safety systems. Of particular interest for our purposes is the fact that an estimate of the visual, cognitive, and motor workload imposed by the various devices was provided. This is the only task analysis information in hand regarding the incab devices rather than driving tasks per se.

Data Type: $\quad$ Formal analysis.

Task Listing: $\quad$ A sample trip recorder task description is included (see Figure 3.1). None others per se. In Table 3.1, a taxonomic phrase is used to indicate the nature of a device display, controls, and the processing functionality.

Task Structure: $\quad$ See Figure 3.1.

Driver: $\quad$ No driver sample described.

Timeline Analysis: None.

Driver Information

Needs:

None explicitly described. Each in-cab device provide information specific to its purpose.

Operational

Validity: N/A.

Driving

Conditions: $\quad$ Table 3.2 reports workload estimates imposed by truck activity (e.g., moving forward, parked, stopped, backing). 
FIGURE 3.1. EXAMPLE TASK SEQUENCE WITH IN-CAB TRIP RECORDER [SOURCE: BURGER, SMITH, ZEIDMAN, 1989].

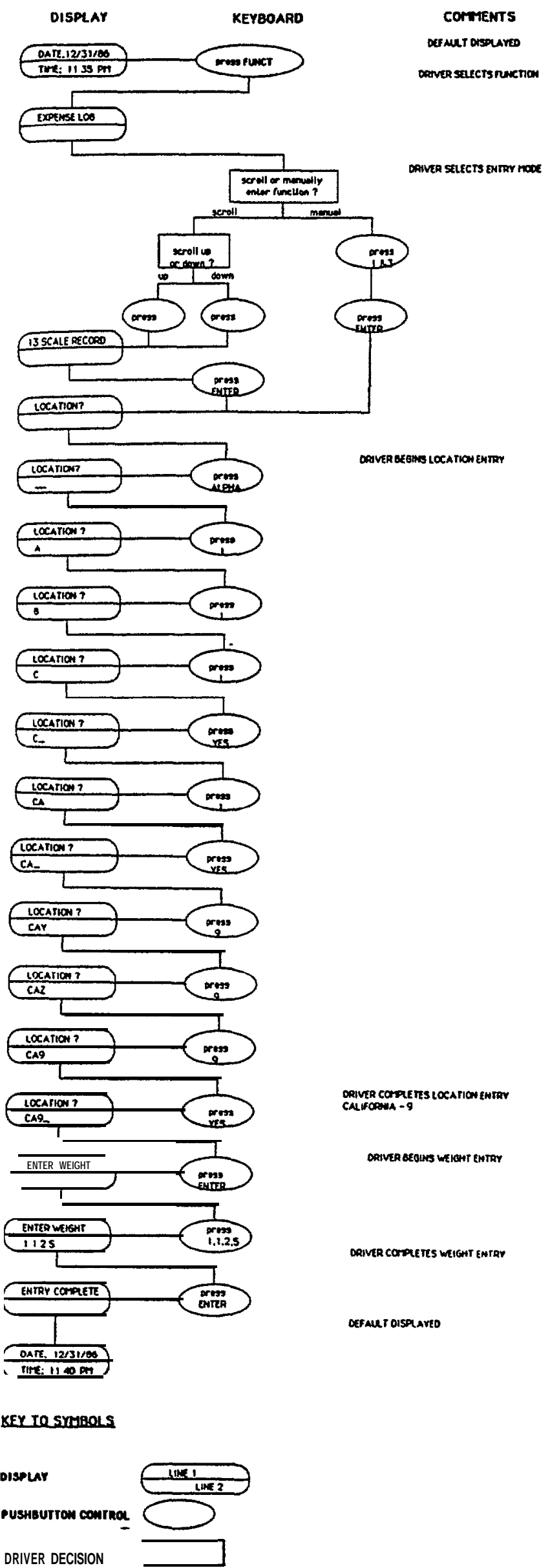


TABLE 3.2. IN-CAB DISPLAY \& CONTROL DEMANDS AS A

FUNCTION OF VEHICLE STATUS,

[SOURCE: BURGER, SMITH, \& ZEIDMAN, 1989].

\begin{tabular}{|c|c|c|c|c|}
\hline \multirow[t]{2}{*}{ IN-CABDEVICE } & \multicolumn{2}{|c|}{$\begin{array}{l}\text { DEVICE DISPLAY } \\
\text { DEMANDS }\end{array}$} & \multicolumn{2}{|c|}{$\begin{array}{l}\text { DEVICE CONTROL } \\
\text { DEMANDS }\end{array}$} \\
\hline & LOW & $\mathrm{HIGH}$ & LOW & $\mathrm{HIGH}$ \\
\hline \multicolumn{5}{|l|}{ SIMELE/MTEGAATED DISPLAT } \\
\hline EASY WEIGH & PKO & N/A & PKD & N/A \\
\hline CHARLIE & FWD & N/A & FWD & N/A \\
\hline FUEL TACH & FWD & N/A & N/A & N/A \\
\hline CARGOSAVERII & Fwo & N/A & PKD & N/A \\
\hline MODEPRO IV & FWD & N/A & FWD & N/A \\
\hline MODEPROV & FmD & N/A & FWD & N/A \\
\hline DB212 DIGITALIBAROGRAPH & FNo & N/A & FWD & $\mathrm{N} / \mathrm{A}$ \\
\hline ETEC ॥ & N/A & N/A & N/A & N/A \\
\hline DIGTAL TEMP CONIROL SYS. & & & & \\
\hline ARGOEDM & FWD & N/A & FWD & N/A \\
\hline T MACS & FWD & $\mathrm{N} / \mathrm{A}$ & & N/A \\
\hline ELECTRONIC DASH & FWD & N/A & FWD & N/A \\
\hline AUDIT & $?$ & $?$ & ? & $?$ \\
\hline ELECTRONIC DASHBOARD & $?$ & $?$ & $?$ & $?$ \\
\hline DDEC II & FWD & N/A & FWD & N/A \\
\hline VIP & FWD & N/A & N/A & N/A \\
\hline \multicolumn{5}{|l|}{ IEXT COMHANCATION SYSTEA } \\
\hline MDI 7031 & N/A & FWD & STP & N/A \\
\hline MDI 9031 & FWD & FWD & FWD & STP \\
\hline \multicolumn{5}{|l|}{ VEHICLE IMFONAATION SYSTEH } \\
\hline FMSS 1330 (BASIC) & N/A & STP & STP & STP \\
\hline FMES1330 (OPTIONAL) & N/A & STP & STP & STP \\
\hline CADEC 100 & FWD & N/A & N/A & $\mathrm{N} / \mathrm{A}$ \\
\hline CADEC $200 \& 300$ & FWD & STP & FWD & STP \\
\hline FLEET DATA MASTER & FWD & N/A & FWD & STP \\
\hline UNIPARS & STP & N/A & STP & $\mathrm{N} / \mathrm{A}$ \\
\hline SILENT 1000 & FWD & STP & FWD & STP \\
\hline TRIPMSTR (WIKEY INPUT) & N/A & N/A & N/A & N/A \\
\hline TRIPASTR ( $w$ DAIVER INPUT) & N/A & STP & STP & STP \\
\hline RRIPHSTR ( W DAIVER KEY) & FWD & STP & FWD & STP \\
\hline DATA-com & $?$ & $?$ & $?$ & $?$ \\
\hline SYSTEM 7000 & FWD & STP & FWD & STP \\
\hline DRIVER INFORMATION SYS. & FWD & FWD & FWD & STP \\
\hline TC-I & FWD & STP & FWD & STP \\
\hline ELECTRONIC RECORDER & N/A & N/A & N/A & N/A \\
\hline MOG & N/A & STP & N/A & STP \\
\hline AL100 & N/A & STP & N/A & STP \\
\hline \multicolumn{5}{|l|}{ NAVIGATION SYSTEMS } \\
\hline TRUCK TRACKER & $?$ & FWo & $?$ & FWD \\
\hline NAV-COM & $?$ & FWD & $?$ & FWD \\
\hline OMNIRACS & $?$ & FWD & $?$ & FWD \\
\hline ETAK NAVIGATOR & $?$ & FWD & FWD & FWD \\
\hline \multicolumn{5}{|l|}{ TRACKING SYSTEMS } \\
\hline ross & N/A & STP & N/A & STP \\
\hline II monnow VTS & N/A & STP & N/A & STP \\
\hline ROCKWELL POSITIONIHS SYS & $?$ & STP & $?$ & STP \\
\hline \multicolumn{5}{|l|}{ VEHICLE SAFETY SYSTEMS } \\
\hline CAR VISION SYSTEM 9300 & STP & $\mathrm{BCK}$ & STP & N/A \\
\hline MODEL 750 & STP & BCK & STP & N/A \\
\hline AUTOMOTIVE WATCHCAM & $?$ & BCK/FWD? & $?$ & $N / A$ \\
\hline CARDAR & $?$ & BCK & $?$ & N/A \\
\hline RETRO-GUARD & STP & N/A & STP & N/A \\
\hline TATTLE TALE & STP & N/A & $?$ & $?$ \\
\hline EBS 1013 & STP & N/A & STP & N/A \\
\hline EBS 3060 & STP & N/A & STP & N/A \\
\hline BACK SENSOR & STP & N/A & STP & N/A \\
\hline EAGLE EYE & STP & N/A & $?$ & N/A \\
\hline
\end{tabular}

KEY TO ABREVIATIONS

FWD = MOVING FORWARD ONROAD

STP $=$ STOPPED ON IOFF ROAD

PKD=PARKED

BCK • BACKING

N/A $=$ CONTROLDISPLAY NOT AVAILABLE 
Criteria for

Safety Thresholds: None explicitly given. Refers to glance frequency data from Antin, Dingus, Hulse, and Wierwille (1986) and indicates the strong relation between number of glances and percent trials where a lane crossing occurred.

Usefulness

of Data:

Risk Taking

Behavior:

Reviewer:

Ellingstad, V. S. (1970). A driving task analysis. In V. Eliingstad, Iniurv control in traffic safetv (pp. 176 - 200).

Data Type:

Task Listing:

Task Structure: None given.

Driver:

Timeline Analysis: None.

Driver Information

Needs:

\section{Operational}

Validity:
As a first approximation to the workload imposed by in-cab devices of the type to be evaluated by the workload protocol, this report has extremely high relevance.

Not discussed.

L. Tijerina
Formal analysis.

A listing of driver tasks (rather than driving) is given:

- $\quad$ search and scan tasks (number and nature of inputs);

- $\quad$ perceptual task (identification and recognition; relating perceived information to information stored in memory); decision task (risk taking and decisions to engage or not engage in a maneuver);

- $\quad$ physical response (wheel movement to control placement on roadway; accelerator and brake pedal movements to control speed)

None specified.

This chapter describes driver processes in general terms. For example, search and scan tasks make use of roadway geometry, traffic density, surface condition, and rate of traffic flow. Car following requires the perception of a gap between own car and leading car, cues for velocity. No further level of detail is provided.

Unknown. This is a formal analysis, not an empirical one. May apply equally to all vehicular control (e.g., car, truck, bus, tank, etc.). 


\section{Driving}

Conditions: $\quad$ None specified.

Criteria for

Safety Thresholds: None given.

Usefulness

of Data:

Not particularly useful for workload purposes. The driver tasks are apparent; their nature is not..

Risk Taking

Behavior:

Nothing of note mentioned.

Methodology: $\quad$ Essentially a literature review and synthesis.

Reviewer: $\quad$ L. Tijerina

Noy, Y. I. (1990). Selective attention with auxiliary automobile displays. Proceedings of the Human Factors Society 34th Annual Meeting (pp. 1533 - 1537).

Noy, Y. I. (February, 1990). Attention and performance while driving with auxiliarv in-vehicle displays (Report No. TP 10727). Ottawa: Transport Canada.

(Note: These two reports cover the same experiments and so are discussed together).

Synopsis:

Data Type:

Task Listing:

Task Structure: None given.

Driver:

Timeline Analysis: None.

Driver Information

Needs:
This was a simulator study of tbe driving performance effects of a visual search task (Perception) and a memory task (Sternberg paradigm), both administered via a CRT and responded to via left-foot presses of one or the other of two switches.

Moving base simulator study.

Driving task was to drive a car over a winding road and maintain constant lane position and follow a lead truck at a constant headway of 50 meters (3 seconds of headway at a speed of $60 \mathrm{kph}$ ). Driving task difficulty was assessed through variations in radius of curvature.

A total of thirty (30) healthy male and female (college) student volunteers. None were truck drivers and the simulator was not a truck simulator.

None described as part of the experiment. 
Operational

Validity:

Driving

Conditions:

Criteria for

Safety Thresholds: Primary driving measures of time to lane crossing (TLC), standard deviation

\section{Usefulness}

of Data:

\section{Risk Taking}

Behavior:

Reviewer:

Unknown. (Undated). Evaluation of high-technology driver displavs. Interim briefing on progress of Task 4.2, Evaluation of High-Technology Driver Aids.

Data Type: $\quad$ Formal analysis. of lane position, standard deviation velocity, and headway all affected by auxiliary tasks.

The author describes the auxiliary tasks as similar to those involved in complex in-cab device interactions such as navigation displays (for perception task). Relevance of memory task not described. Neither task can be judged an acceptable (or unacceptable) analogue of in-cab device interactions without a taxonomic basis. While visual search is considered part of map reading, a taxonomic basis was not provided and so it is difficult to determine, for example, just how similar a Sternberg task is to land navigation device interaction. Driving performance degraded due to intrusion by auxiliary tasks, but only the perception task data is presented by name.

One of the studies reported attempted to increase the utility for focusing on the CRT auxiliary tasks by monetary incentive. No modification (i.e., even more extreme driving degradation than previously observed) in workload allocation was found. Author interprets this to mean that attention allocation is automated for experienced drivers and resistant to short-term reallocation. I disagree and suggest that it would have been better, to test the utility hypothesis, to try and entice the subjects in the direction of the primary task, given that they already showed a propensity to let primary task performance degrade. Relevance of this simulator study to real driving needs validation because the allocation strategies may indeed be quite different.

Simulated two-lane roads taken from a roads library were used. Daylight, low-to-no traffic, good weather conditions simulated. Straight and circular arc road segments were sequenced differently for different trials to insure unique road geometry but essentially the same driving conditions and demands.

This study, while it does only indirectly address the issue of driver workload for driving tasks, does indicate measures which appear sensitive to intrusion effects (at least for the auxiliary tasks and range of difficulties used).

None.

L. Tijerina 
Task Listing: None.

Task Structure: $\quad$ A data collection taxonomy is offered to characterize in-cab devices (See Table 3.3.), but its utility is unclear at this point.

Driver: $\quad$ None.

Timeline Analysis: None.

Driver Information

Needs:

Indirectly given by sensory system used when interacting with a device.

Operational

Validity: Not applicable.

Driving

Conditions: $\quad$ None.

Criteria for

Safety Thresholds: None.

Usefulness

of Data:

Such a characterization may have value in estimating the workload imposed by a device. It should be explored further in later phases of this project.

Risk Taking

Behavior: None.

Reviewer: $\quad$ L . Tijerina

Verwey, W. B. (1991). Towards guidelines for in-car information management: Driver workload in specific driving situations (Report No. IZF 1991 C-13). The Netherlands: TNO Institute for Perception.

Synopsis:

This was a workload assessment study using three secondary tasks. One was a VISUAL task which involved the subject saying "Yes" (in Dutch) if a twodigit number appeared on a visual display mounted on the dashboard; two $x x^{\prime}$ 's were displayed when the numerals were not. AVISUAL-COGNITIVE task involved adding 12 to the visually displayed number (when presented) and saying the result out. A COGNITIVE ONLY task was the same as the preceding task but the two-digit number was presented auditorily (i.e., no visual component). Eye glance measures and driving measures (steering wheel activiation rate, speed and its standard deviation, time to merge, distance required to merge, speed when merging had finished, time of braking before an intersection, distance of braking before an intersection, and speed while braking before an intersection) were also measured. 

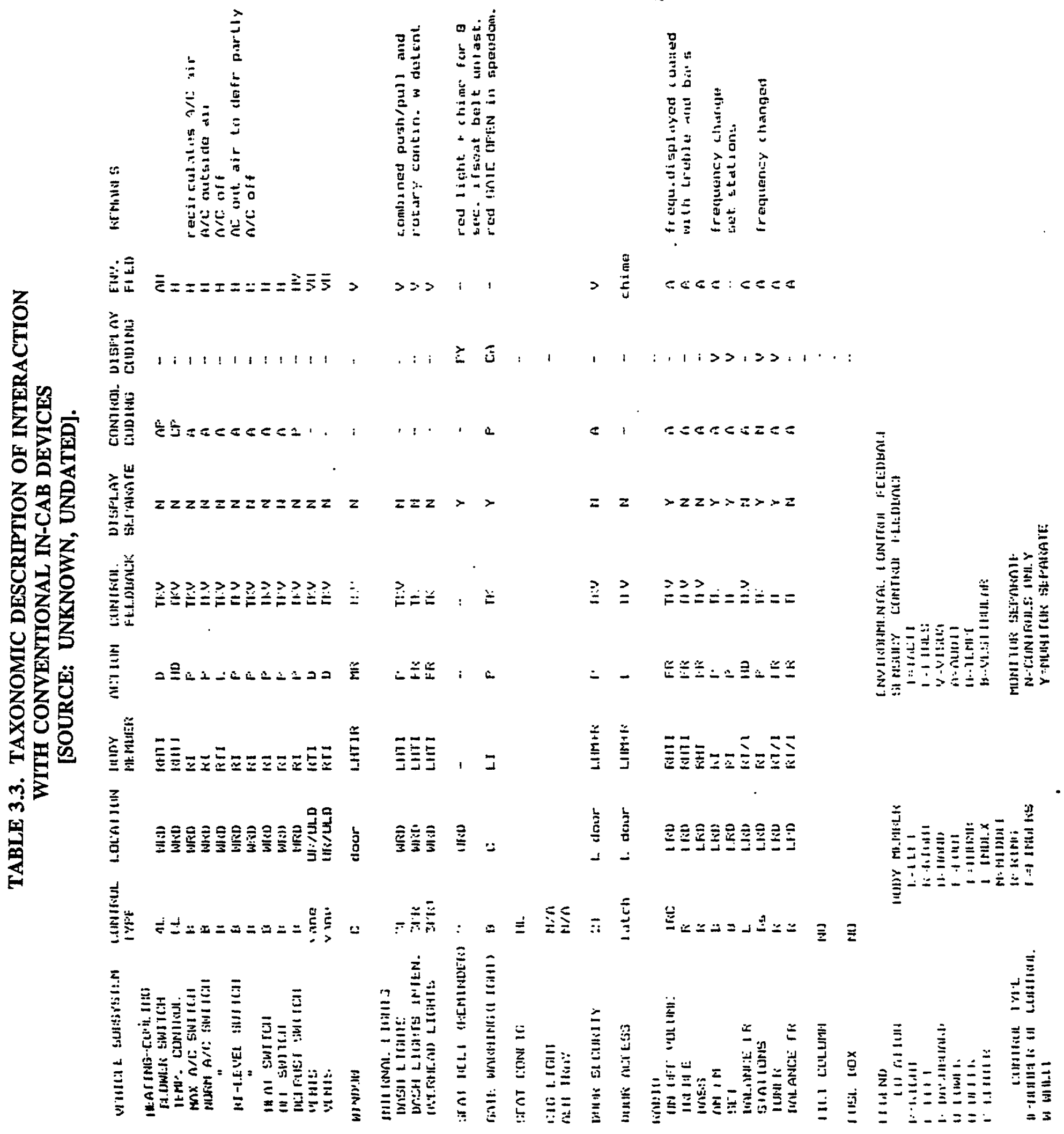
Data Type: $\quad$ On-the-road data collection in a Volvo 240 station wagon.

Task Listing: $\quad$ No driving tasks listed as such. Drivers drove a route which included merging/exiting, a straight motorway, a roundabout, a simple right turn, a complex turn left including crossing a bicycle lane, and straight rural road where bicycles are allowed.

Task Structure: None.

Driver: $\quad$ Twelve male and twelve female car drivers. Half of each group was inexperienced (e.g., licensed less than one year and had driven less than $10,000 \mathrm{~km}$ ) and the other half was experienced (e.g., licensed for five or more years and had driven more than 10,000 km per year). Mean age $=28$ yrs. for experienced drivers and 21 yrs for inexperienced drivers.

Timeline Analysis: None.

Driver Information

Needs:

None specified

Operational

Validity:

High, for automobile driving.

Driving

Conditions: Incomplete description. Driving done atthree different times of the morning and three different times of the afternoon to capture differences in traffic density.

Criteria for

Safety Thresholds: None given.

Usefulness

of Data:

Indicates that visual and cognitive workload while driving can be assessed through steering wheel actuation rate and mirror glance frequency. A significant effect of experience was found on secondary cognitive task performance. Visual load affected all drivers. Author admits further research needed for verification.

\section{Risk Taking}

Behavior:

None mentioned.

Methodology: $\quad$ Secondary task, visual allocation, primary driving measures.

Reviewer: $\quad$ L. Tijerina 


\section{Allen, T.M., Lunenfeld, H., and Alexander, G.J. (1971). Driver information needs. Highway Research Record, No. 366. Washington, DC: Highway Research Board. pp. 102-115.}

Note:

Synopsis:

$\operatorname{Method(s):~}$

Task Listing:

Safety

Criticality:

Time Line

Analysis:

Detailed Human

Performance

Descriptions:
This article summarizes a portion of a larger report. Because of the summary nature of the article, it is difficult to assess the usefulness of the source data for the truck driver workload project. It is recommended that the complete report be acquired and reviewed, if it can be obtained. Reference: Alexander, King and Warskow. Development of Information Requirements and Transmission Techniques for Highway Users. AIL, Division of CutlerHammer. Nov. 1967.

A driver task analysis was performed to enable information required by drivers to be identified. The task analysis divided driving into several interrelated subtasks which are hierarchically organized. Tasks differed in time scale, "primacy" (i.e., priority) and in the level of cognitive activity required. A graphical model of the driving task (See Figure 3.2) was presented to illustrate the relationships.

Three major task divisions were identified: (a) control or micro-performance tasks (i.e., steering and speed control), (b) guidance or situational performance tasks (i.e., responding to specific situations such as weather, traffic or road conditions) and (c) navigation or macro-performance tasks (e.g., pre-trip planning, direction finding). A sample of the task analysis is attached.

Eight categories of driver information needs were identified: (1) vehicle micro-performance, (2) advisory, restrictive or inhibitory (ARI) microperformance, (3) road micro-situational, (4) traffic situational, (5) ARI situational, (6) service macro-performance, (7) directional macro-performance and (8) ARI macro-performance. Table 3.5 presents the information needs for the directional macro-performance category.

Analysis based on observations of drivers.

Primary tasks, but not well defined in the article. See Table 3.4.

None.

The task listing appears to be sequential in nature, but no frequency or duration data are supplied.

Perceptual, cognitive and motor requirements are described, but not with the detail needed for this project. No task loading data. 
FIGURE 3.2. DESCRIPTION OF DRIVING

[SOURCE: ALLEN, ET AL.; 1971]

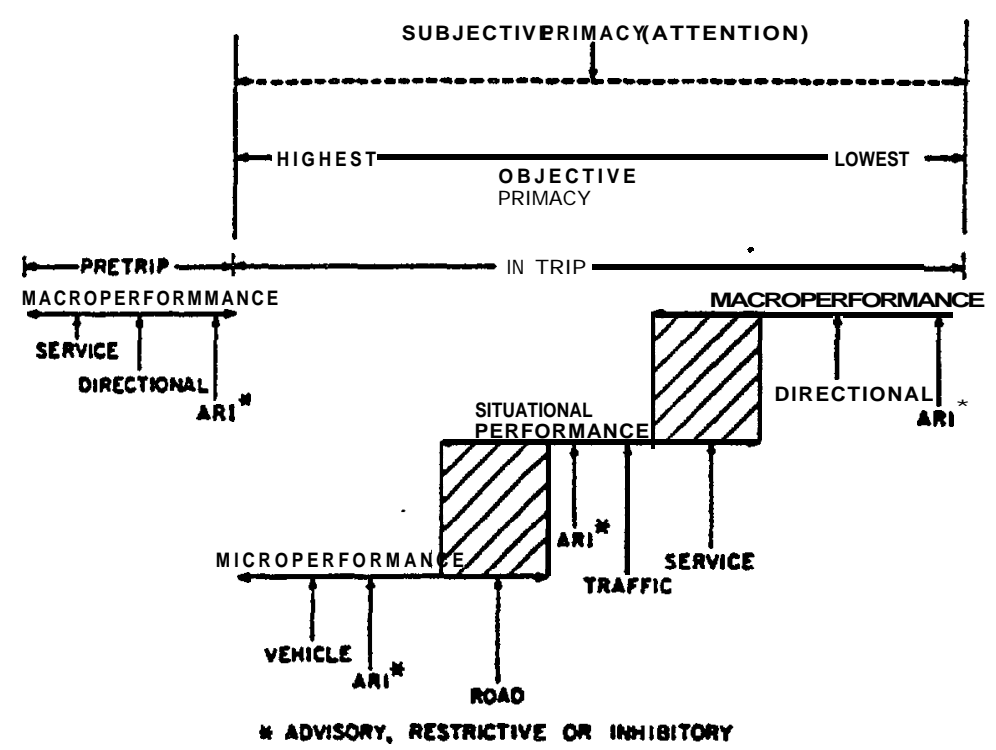


TABLE 3.4. VEHICLE CONTROL TASK ANALYSIS [SOURCE: ALLEN, ET AL.; 1971]

\begin{tabular}{|c|c|c|c|c|c|c|c|c|c|c|c|}
\hline \multicolumn{2}{|c|}{ Hew } & \multirow{2}{*}{$\begin{array}{l}\text { Bomed } \\
\text { (maph) }\end{array}$} & \multirow{2}{*}{$\begin{array}{l}\text { Roed Cooditione } \\
\text { and Trane } \\
\text { Dparmies }\end{array}$} & \multicolumn{3}{|c|}{ Driwar Dphemen } & \multirow{2}{*}{$\begin{array}{l}\text { Yehicle } \\
\text { Reapoesee }\end{array}$} & \multirow{2}{*}{$\begin{array}{l}\text { Fened: } \\
\text { back: }\end{array}$} & \multirow{2}{*}{$\begin{array}{c}\text { Dletrace } \\
\text { Howe }\end{array}$} & \multicolumn{2}{|c|}{ Remerto } \\
\hline No. & Elappend & & & Purcaption & Coeplition" & Rospones & & & & 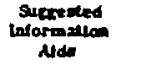 & Comonenter \\
\hline \multirow[t]{2}{*}{14} & $\infty \overline{000}$ & $\bar{\infty}$ & Farmen loc & rentex ar & 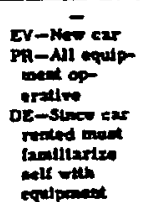 & 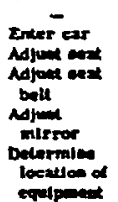 & Non $=$ & $v \tilde{r}$ & $=$ & 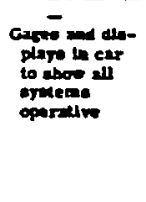 & Prwetitis: \\
\hline & & & & All & 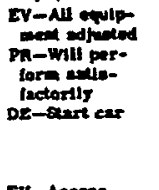 & 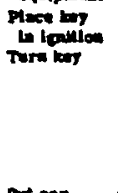 & Car & $v / T / A$ & - & 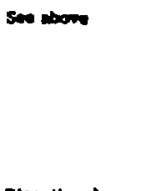 & 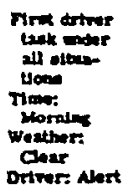 \\
\hline \multirow[t]{2}{*}{88} & $\infty 000$ & 20 & 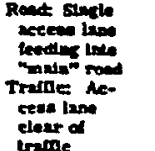 & 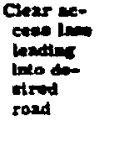 & 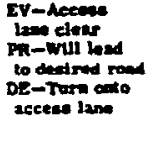 & 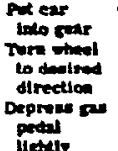 & 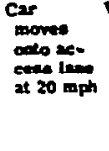 & $v / T / x$ & $\begin{array}{l}\text { Pedre: } \\
\text { irtan: }\end{array}$ & 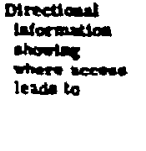 & 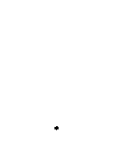 \\
\hline & $\infty 00.5$ & 20 & 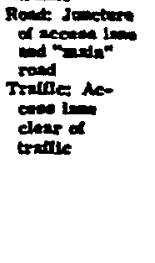 & Theld alon & 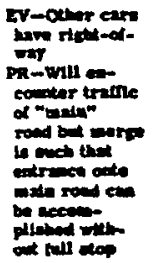 & 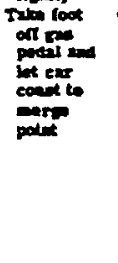 & Car & $v / T / \pi$ & - & 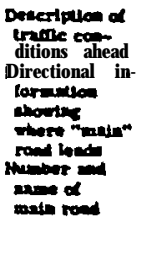 & 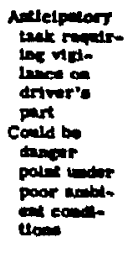 \\
\hline
\end{tabular}


TABLE 3.5. DIRECTIONAL INFORMATION NEEDS

[SOURCE: ALLEN, ET AL., 1971]

\begin{tabular}{|c|c|c|c|}
\hline then & 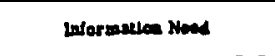 & Doflueluce & 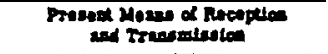 \\
\hline I & 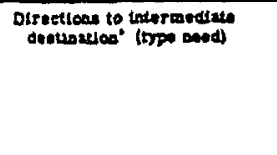 & 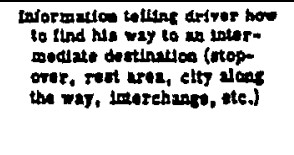 & 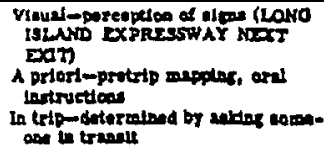 \\
\hline 2 & 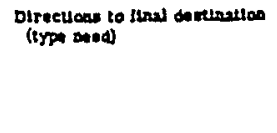 & 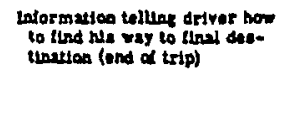 & 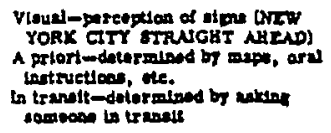 \\
\hline 3 & 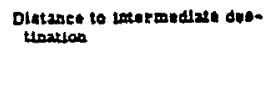 & 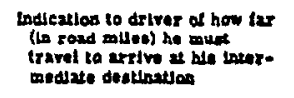 & 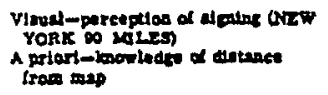 \\
\hline 4 & $\begin{array}{l}\text { Nummete route; onetall } \\
\text { liype noeds }\end{array}$ & 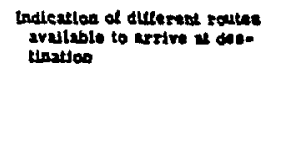 & 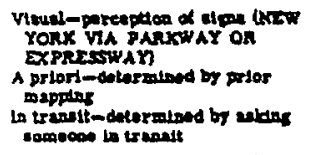 \\
\hline 3 & 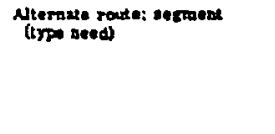 & 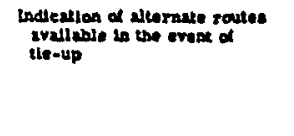 & 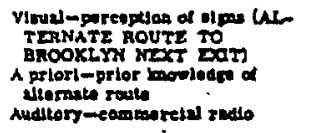 \\
\hline : & 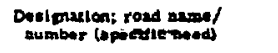 & 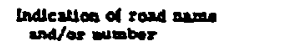 & 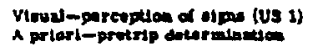 \\
\hline 7 & 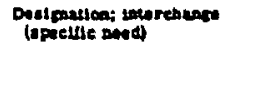 & 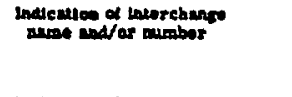 & 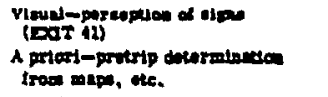 \\
\hline 1 & 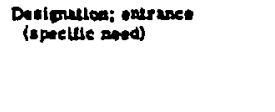 & 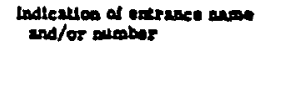 & 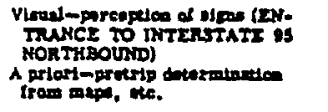 \\
\hline ? & 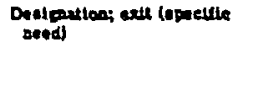 & 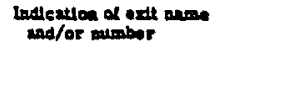 & 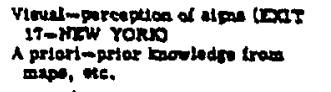 \\
\hline 10 & 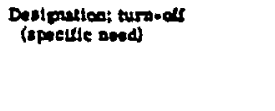 & 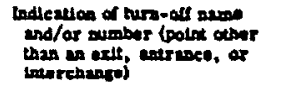 & 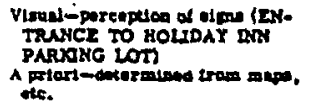 \\
\hline t1 & 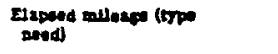 & 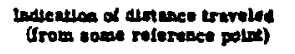 & 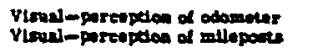 \\
\hline 12 & 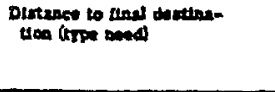 & bodleation of milles to so to & 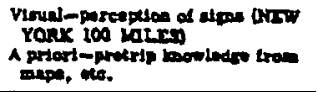 \\
\hline
\end{tabular}


Subjects:

Driving

Conditions:

Operational

Validty:

Risk Taking

Behavior:

Usefulness

of Data:

Reviewer:

Subject characteristics not stated.

Urban freeways \& one long distance trip. Actual conditions not stated.

Appears high for car drivers; unclear for truck drivers.

Not discussed.

This summary article lacks the detail needed to be useful in this project.

Stein, A.C., Parseghian, Z., Allen R.W. and Hayes, J.T. (1990). The Development of a LowCost Portable System for the Detection of Truck Driver Fatigue. Proceedings of 34th Annual Association for the Advancement of Automotive Medicine, October 1-3, 1990, Scottsdale, AZ.

Synopsis:

The status of efforts to develop a portable, low-cost device for evaluating fatigue in commercial truck drivers was presented in this conference paper. The development focused on the use of a Sub-critical Tracking Task in a driving simulation to detect fatigue-induced driving impairment.

The device developed was a static, PC-based, part-task truck simulator which included included an instrument panel (IP), steering wheel, pedals and seat. The Sub-critical Tracking Task, described as "moderate workload," was incorporated into the longitudinal speed and the lateral placement subsystems of the vehicle. A secondary task (not described in the paper) was incorporated in the truck side "mirrors."

Seventy professional truck drivers participated in 200 demonstration runs of 20 minutes duration. Experimental runs were conducted either before or after the driver's work shift. Independent variables were: before/after work shift and run segment (10 segments per run). Dependent variables used were: (a) mean response time to secondary task, (b) RMS of response time, (c) mean lane position, (d) mean vehicle speed, (e) RMS of speed, (f) RMS of steering wheel activity, (g) RMS of gas pedal activity and (h) RMS of lane position. An ANOVA indicated no significant differences between means for either the mean response time or lane tracking variability. For all other measures, significant before/after effects were observed. Interaction effects between before/after and 2-minute run segments were observed for all variability measures (except lane tracking) and also for mean speed.

Method(s): $\quad$ Simulated truck driving with secondary task (not specified). 
Task Listing: None.

Safety

Criticality: None.

Time Line

Analysis:

None.

Detailed Human

Performance

Descriptions:

None for driving tasks. Data presented was limited to four graphs of primary task performance (see Figure 3.3).

Subjects:

Seventy truck drivers employed by a national trucking company. No data on subject characteristics was given.

Driving

Conditions:

Unspecified levels of simulated gusting wind and speed perturbations. Other conditions were not described.

Validity

Issues:

Realism of driving task. Mean response time and RMS of lane position were reported to be consistent with full scale tests.

Risk Taking

Behavior:

Not addressed.

Usefulness

of Data:

This article is of little help in the development of a task analysis. However, potential measures of primary task performance are suggested which could be used in simulated or actual driving later in the project.

Reviewer: $\quad$ S. Kiger 
FIGURE 3.3. DRIVING PERFORMANCE DATA

[SOURCE: STEIN, ET AL., 1990].

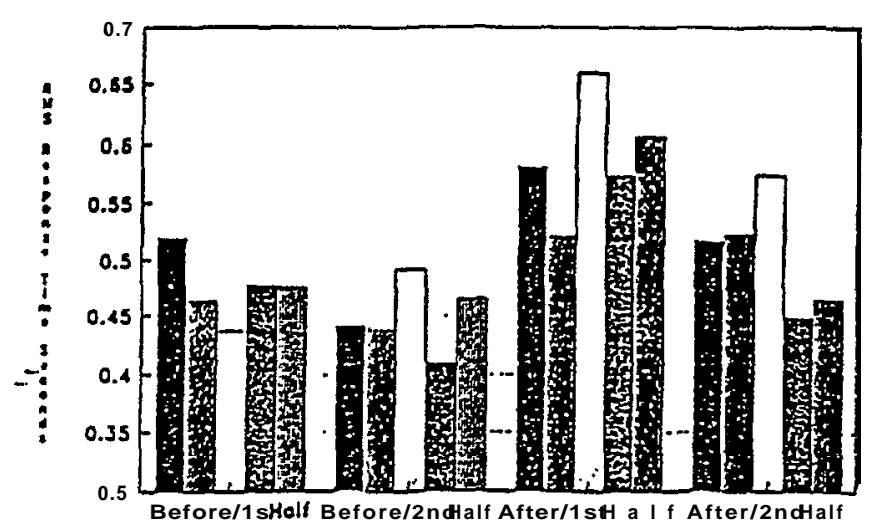

High Risk DrivelProject RMSResponse Time

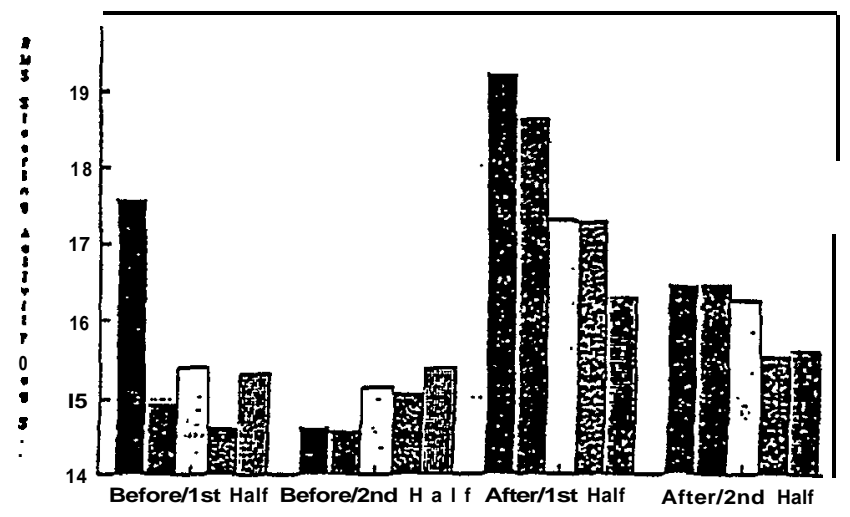

High Risk Driver Project - RMS Steering Activity

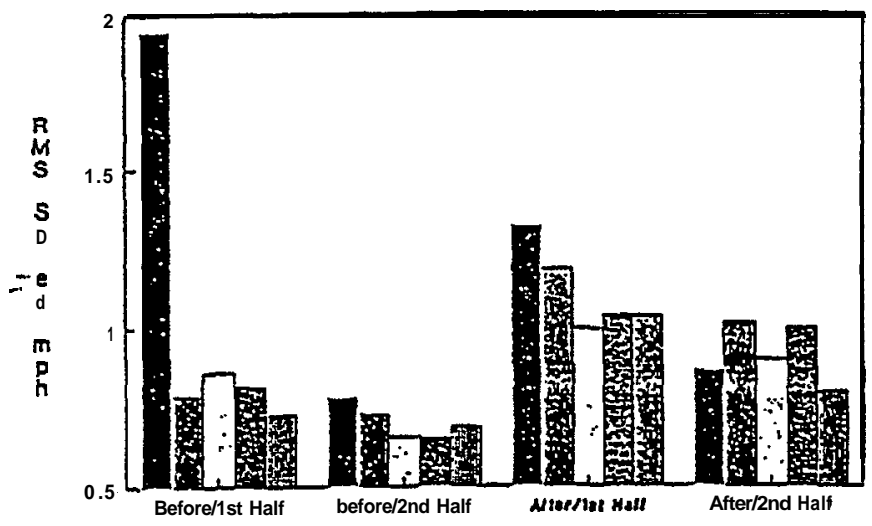

High Risk Driver Project - RMS Speed

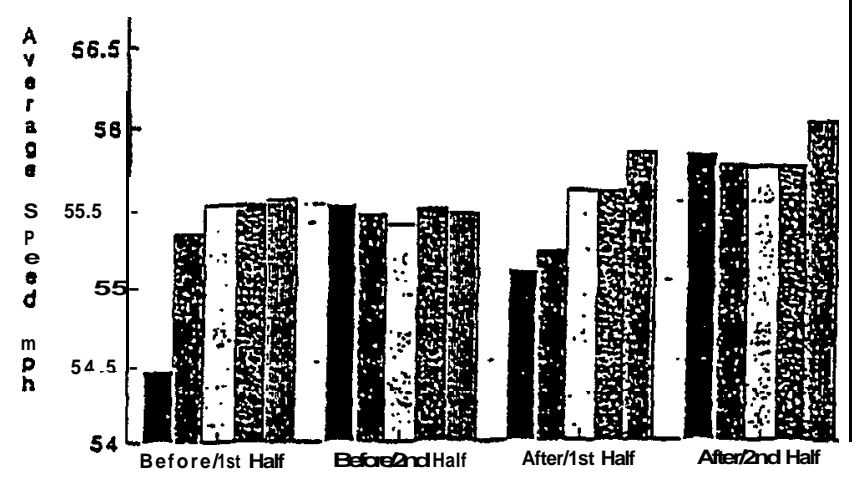


Taoka, G.T. (1990). Duration of Drivers' Glances at Mirrors and Displays. LE_Journal, October 1990, pp. 3539.

Synopsis:

Method(s):

Task Listing:

Safety

Criticality:

Time Line

Analysis:

Detailed Human

Performance

Descriptions:

Subjects:

Driving

Conditions:

Validity

Issues:

Risk Taking

Behavior:

Usefulness

of Data:

Reviewer:
Log-normal probability distributions were fit to experimental data published by Wienville and Rockwell for visual tasks performed by car drivers. The visual tasks included looks to: the radio, left mirror, right mirror, speedometer, temperature gauge, defroster and roadway name sign. Percentile estimates of glance durations were calculated for each task based on the distributions fit.

Statistical data analysis.

No task analysis performed.

None.

None.

None.

No driving experiments conducted.

None.

Significant differences may exist between truck and car driver visual allocation.

Not discussed.

No task analysis was performed. Estimates of driver glance durations for several visual tasks typically performed by car drivers are presented. Whether these data are applicable truck drivers is not known, as there are significant differences between truck and car driving (e.g., vibration, cab layout, etc.)

M.J. Carnot \& S. Kiger 


\section{Hale, A.R., Quist, B.W., and Stoop, J. (1988). Errors in routine driving tasks: a model and proposed analysis technique. Ergonomics. Vol. 31. No. 4, pp. 631-641.}

Synopsis:

Method(s):

Task Listing:
An occupational safety model for the analysis of "individual behavior in the face of danger" was applied to driving to assist in identifying the causes of a certain class of accidents at a specific intersection in the Netherlands. At this intersection, a large number of drivers from the minor road were struck, while crossing or turning left, by fast-moving traffic on the second lane of a divided major highway. A key characteristic of these accidents was the failure of the at-fault driver to detect the oncoming car. The overall objective of the study was to identify accident countermeasures for the site.

The occupational safety model used was a hierarchical model of performance (i.e., input, processing and output) at three levels of functioning: skill based, rule based and knowledge based functioning (see Figure 3.4). From an analysis of accident data and on-site observations (not described), a proposed model of "ideal" behavior for drivers approaching on the minor road at the intersection in question was presented (see attached). Using the "ideal" behavior model, a discussion of driver errors at the intersection was included, Interviews with drivers on the minor road at the intersection were conducted to obtain data to validate the proposed model, but he data obtained were "unreliable" and could not be used.

Analysis.

No detailed listings. Only a very general (i.e., vague) listing of driver tasks at an intersection were presented.

1. Approach crossroad and observe (from signboards, road layout + memory of where in the journey he is) that Winsum crossroad is ahead.

2. Select appropriate Krimpenerwaard crossing programme.

3. Slow speed (and change gear).

4. Look left.

5. If traffic is approaching, and there is no time to cross safely in front of it, then stop and give way (switch to more conscious level of control).

6. If no traffic is approaching, or there is time to cross safely in front of it, then accelerate (and change gear).

7. Slow speed (and change gear). .

8. Look right.

9. If traffic is approaching, and there is no time to cross safely in front of it, then stop and give way (switch to more conscious level of control).

10. If no traffic is approaching, or there is time to cross safely in front of it, then accelerate (and change gear).

11. Reselect normal road programme. 
FIGURE 3.4. MODEL OF INDIVIDUAL BEHAVIOR IN THE FACE OF DANGER [SOURCE: HALE, QUIST, AND STOOP, 1988].

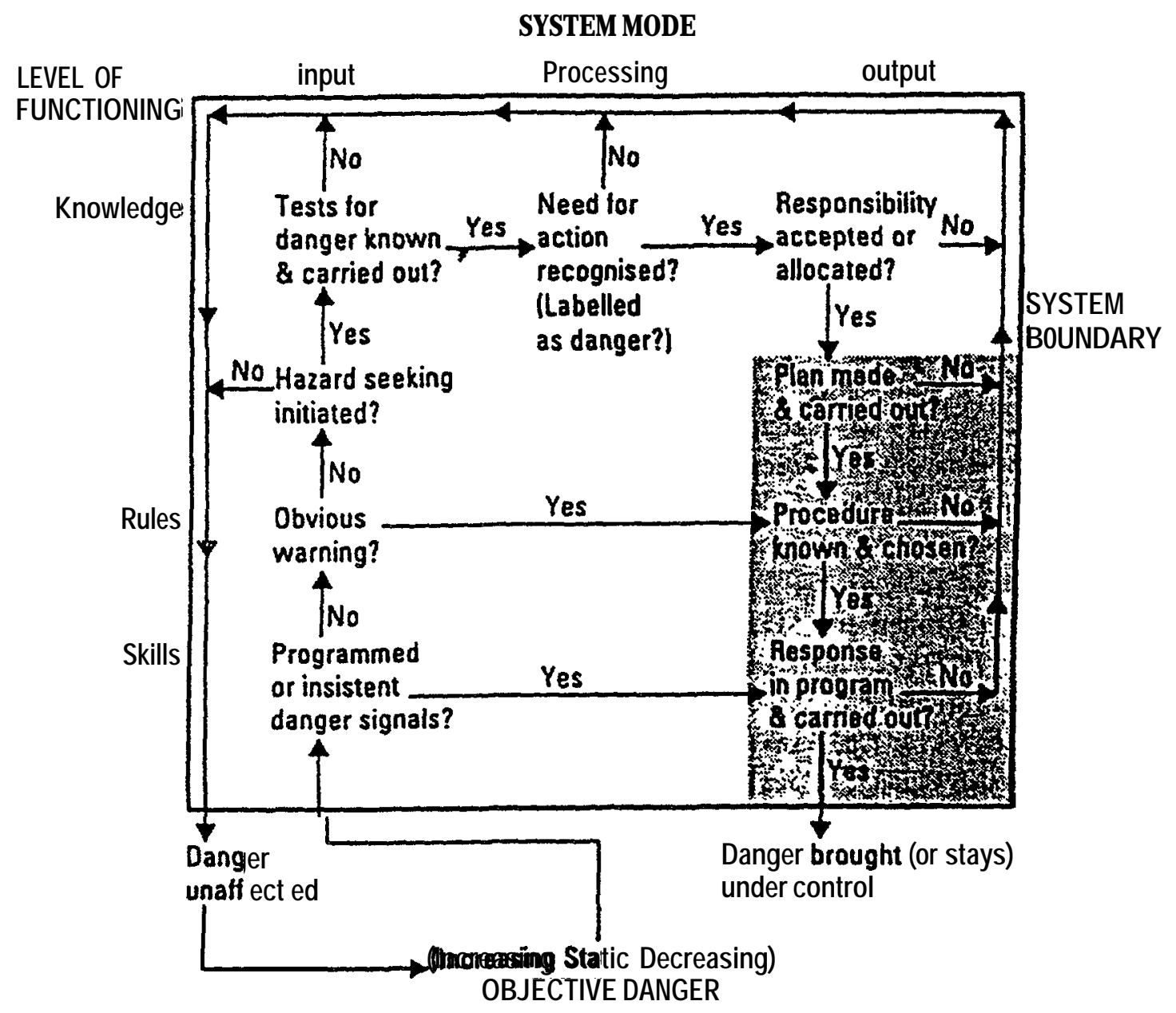


Safety

Criticality: None.

Time Line

Analysis:

None.

Detailed Human

Performance

Descriptions:

None.

Subjects: Not described.

Driving

Conditions:

Not described.

Validity

Issues:

Proposed model was not validated.

Risk Taking

Behavior:

The article stated that a person engages in risk assessment at the knowledgebased level of functioning. Errors and accidents occurring here would involve such factors as ignorance of risk, conscience preference for speed over safety or preoccupation with one problem at the expense of others.

The authors suggested that there is "scope" for risk homeostasis at both the rule-based and knowledge-based levels of functioning.

Usefulness

of Data:

Not useful. No task analysis presented. Insufficient detail in proposed model. Model not applicable to U.S. driving. Model not validated.

Reviewer: $\quad$ M. J. Camot \& S. Kiger

Drory, A. (1985) Effects of rest and secondary task on simulated truck-driving task performance. Human Factors, 27, 201-207.

Data Type: Modified Redifon light motor vehicle simulator.

Task Listing:

Primary task: $\quad$ Basic driving

Secondary task: $\quad$ Light-canceling vigilance task with an average frequency of one light every $40 \mathrm{sec}$.

Secondary task: $\quad$ Voice communication four times during each 15 minute block. Driver reads the two least significant digits on odometer.

Task Structure: None. 
Driver:

Timeline Analysis: None.

Safety Criticality: Driver fatigue hypothesized to be safety critical.

\section{Driver Information}

Needs:

\section{Operational \\ Validity:}

\section{Driving}

Conditions:

Criteria for

Safety Thresholds:

Usefulness

of Data:

\section{Risk Taking \\ Behavior:}

Methodology:

Reviewer: years with a large mining firm. leading simulated truck at random intervals. environment"). period every 15 minutes. experiment. secondary task.

Dual-task.

B. Kantowitz
Sixty professional truck drivers. Mean age $=39$. Mean experience $=3$

Driver had to maintain a "reasonable speed." Had to slow down or brake for

Driver validity high. Simulator validity unspecified but probably low (e.g., "appearance of a dimly lit road surrounded by a black, featureless

Simulated night driving for a $7 \mathrm{hr}(12 \mathrm{PM}$ to $7 \mathrm{AM})$ shift. Six minute rest

Tracking error, steering wheel reversals, brake responses and reaction time and control-light response time recorded but not explicitly related to safety. Subjective fatigue checklist administered 15 minutes prior to end of

Results showed that performance and perceived fatigue were significantly higher when the voice communication secondary task was added. Less effect for secondary vigilance task. Extra 30 minute rest period alleviated reported fatigue but did not alter performance. Note that the secondary task was intended to increase driver arousal and improve primary-task performance. While this did occur, these data cannot easily be used as an index of workload because primary-task performance was altered by the insertion of the

Not explicitly mentioned. However, brake reaction time was faster with the secondary vigilance task present which may have implications for risk taking. 
Haight, F. A. (1969?). A mathematical model of driver alertness. XXXXXXXX, 367-378.

Data Type:

Task Listing:

Task Structure:

Driver:

Timeline Analysis:

Safety Criticality:

Driver Information

Needs:

\section{Operational}

Validity:

Driving

Conditions:

\section{Criteria for}

Safety Thresholds:

\section{Usefulness \\ of Data:}

\section{Risk Taking \\ Behavior:}

Methodology:
Automotive driver collision avoidance model (10 parameter).

Collision avoidance task is divided into (i) "Hazard" - danger perceived by driver, (ii) "Scanning Process" - the process of sampling the hazard state, and (iii) "Avoidance Manoeuvre and Risk" - a manoeuvre (e.g., braking) is executed only when the scanned hazard is judged to be above a risk threshold.

"Hazard," "Scanning Process," and "Avoidance Manoeuvre and Risk" are strictly sequential.

Conceptual driver population characterized only in terms of parameter variations that might be used for individual differences.

One example shown that is typical of what might be derived from model outputs.

Model is focused on one accident potential scenario.

Model implicitly identifies limited internal information (e.g., risk threshold) and gross external information (re: hazard).

No validation in this report, but may have evolved since paper was written.

Conditions not specified but some environmental variations are conceptually possible with parameter variations.

This models could yield performance criteria to a very limited extent (e.g., probability that a collision would occur under specified condition variations).

Temporal safety margin and other safety-related distribution could be explored to limited extent using model.

Modeled in terms of danger-hazard perception function, scan rates, risk tolerance.

This model's "minimum scan rate" attentional parameter could be related to outcomes (e.g., accident/no accident); hence, provides an attentional workload measure of potential relevance.

A. Bittner 
Hancock, P. A., Wulf, G., Thorn, D., and Fassnacht, P. (1990). Driver workload during differing driving maneuvers. Accid. Anal \& Prev., 22, 281-290.

Data Type: $\quad$ On the road in automobile.

Task Listing: $\quad$ Right and left turns.

Task Structure: $\quad$ None.

Driver: $\quad 18$ staff from USC. Mean age $=30$.

Task Analysis: None.

Safety Criticality: Motor cycle-automobile crashes occur predominantly when auto makes a left turn into path of oncoming motorcycle.

Driver Information

Needs:

Not specified.

Operational

Validity:

High for automobiles.

Driving

Conditions: $\quad$ Urban streets in Los Angeles.

Criteria for

Safety Thresholds: None.

Usefulness

of Data:

Used both TLX and SWAT subjective mental workload scales. Simple RT probe asynchronous secondary task. (Note: Analysis of probe response error was incorrect.) Eye blinks and head reversals recorded. Results were interpreted as supporting structural interference and resource competition both play an important role in detection failures during left turns.

Risk Taking

Behavior:

Not discussed.

Methodology: Dual task, eyeblinks and head reversals, and subjective rating scales.

Reviewer: $\quad$ B. Kantowitz

Harms, L. (1991). Variations in drivers' cognitive load: Effects of driving through village areas and rural junctions. Ergonomics, 34, 151-160.

Data Type: $\quad$ On-the-road automobile driving in village and rural areas in Sweden. 
Task Listing: $\quad$ Primary driving task. Secondary arithmetic subtraction task.

Task Structure: $\quad$ None.

Driver:

Experiment 1: Nineteen professional drivers from a private salvage organization. Instructed to drive as "they would usually do."

Experiment 2: Fourteen non-professional drivers

Timeline Analysis: None.

Safety Criticality: Inverse relationship found between driving speed and cognitive load. (Note: This replicates Harms 1986 which related cognitive load to safety directly.)

Driver Information

Needs:

Not very specific - only village versus urban driving.

Operational

Validity:

Strong empirical support for secondary-task methodology for automobile driving.

Driving

Conditions:

Urban and rural. Other conditions not specified.

Criteria for

Safety Thresholds: None.

Usefulness

of Data:

Excellent indication of cognitive spare capacity.

Risk Taking

Behavior:

Not formally discussed in article. However, result that "processing demands arising from the traffic environments of village areas and rural junctions were higher and caused a substantial increase in the drivers' cognitive load although the drivers actually reduced their speed in those environments" has implication for risk homeostasis hypothesis.

Methodology: Dual task.

Reviewer: $\quad$ B. Kantowitz

Kuruu, C. N., and Morrow, B. W. (1979?). A causal model for single vehicle accidents. In HS 801979 XXXXXXXX, 535549. 
Data Type: General armchair analysis of single vehicle accidents including, a causal factor taxonomy, and consideration of use of model in countermeasure development.

Task Listing: $\quad$ Accidents are seen as involving one of a number of mutually exclusive tasks:

(i) "Maintaining Course, (ii) "Vehicle Control," (iii) "Vehicle Avoidance,"

(iv) "Obstacle Avoidance," and (v) "Hazard Response" that occur in the context of a specific preaccident maneuver (e.g., Going Straight, Curve Negotiation, etc.).

Task Structure: Drivers accomplish tasks following a sequence of: Search (for situations), Identification (of situation as potential problem), Evaluation (of alternative responses), Decision (selection of response), and Driver Action (making response) that is followed by Vehicle Action.

Driver: $\quad$ Driver is categorized with respect to five causal factor categories: (i) Asleep/Unconscious, (ii) Physical Deficiency, (iii) mental Perception, (iv) Driving Deficiency. These, in turn, are broken down in more detail (e.g., Asleep/Unconscious may arise from Injury/Illness, Fatigue, Alcohol, etc.).

Timeline Analysis: $\quad$ Source suggests timeline of drivers tasks in each situation as described in Task Structure. [It does not indicate frequencies of Task, but suggests use of frequencies of causal factors in selecting countermeasures.]

Safety Criticality: Source does not indicate what tasks are critical from a safety standpoint (though accident analysis using its taxonomy might produce such a list for heavy vehicles).

\section{Driver Information}

Needs:

Model is not explicit with respect to nature of information types and cues.

Operational

Validity:

Driving

Conditions:

Frequencies of some general motor accident features are provided as a context for model (e.g., 95\% of single vehicle accidents involve driving on shoulder at some point).

Criteria for

Safety Thresholds: None explicated.

Usefulness

of Data:

Causal Factor Categorization useful in identifying aspects that need to be specifies in driver workload protocols.

Risk Taking Behavior:

Risk taking behaviors are not addressed as such. 
Methodology: $\quad$ Formal analysis of single vehicle accidents provided a task structure and a causal factor taxonomy.

Reviewer: $\quad$ A. Bittner

MacDonald, W. A., and Hoffman, E. R. (1980). Review of relationships between steering wheel reversal rate and driving task demand. Human Factors, 22, 733-739.

Data Type: $\quad$ Review including on the road, test track, and simulator driving.

Task Listing: $\quad$ N/A

Task Structure: $\quad$ None.

Driver: $\quad$ Not specified.

Timeline Analysis: None.

Safety Criticality: Relationship between steering wheel reversals and task demands depends on the level of task difficulty relative to the driver's capacity to cope with it.

Driver Information

Needs:

Lane width and width of car.

Operational

Validity :

Can't be evaluated without reading the cited studies.

Driving

Conditions: $\quad$ Not specified.

Criteria for

Safety Thresholds: Some implications for driver experience and possible overload.

Usefulness

of Data:

Useful warning of a major limitation in interpreting steering wheel reversal rate. Traditional interpretation that reversals are "a measure of task load" is called into question. Low reversal rate could indicate either low or very high task demand. For high task demands low reversal rate could indicate a decrease in attention to the steering task.

Risk Taking

Behavior:

Not discussed.

Methodology: $\quad$ Analytic evaluation based upon models of attention and capacity.

$\begin{array}{ll}\text { Reviewer: } & \text { B. Kantowitz }\end{array}$ 


\section{Malone, T. B., Krumm, R. L., Shenk, S. \& Kao, H. (1972). Human Factors Criteria for Vehicle Controls and Disulavs (Final Report for DOT-HS-120-1-174)). Alexandria, VA: Essex Corporation (DOT HS-800 742).}

Data Type:

Task Listing:

Task Structure:

Driver:

Timeline Analysis:

Safety Criticality:

Driver Information

Needs:

Validity:

Driving

Conditions:

Criteria for

Safety Thresholds: None given.

\section{Usefulness}

of Data:

None. study.

None.
This study was directed toward developing criteria for standardizing control locations in cars, trucks, and buses using: (i) Control-Display Analyses (e.g., paper studies of commonality, criticality, etc.) as a means of identifying standard control and display panel locations; (ii)_A laboratorv Driving Analog to evaluate three beam control locations; and (iii) An On-track Study to evaluate the effects of familiar and unfamiliar control location. These latter two studies were directed at respectively validating the paper analysis approach and need for commonality in panels.

Driving tasks were characterized only for the two evaluation tasks. [The "Driving Analog" involved placing a beam control to High, medium or low on verbal command while simultaneously tracking three exterior lights with the wheel. "On-track" involved speed of locating controls and displays while simultaneously driving the track course.]

Drivers were identified sparsely in this report. "Six subjects" were identified in the Analog Study and " 16 male and 9 female car drivers" for the On-Track

Source does not indicate what tasks are critical from a safety standpoint.

Information needs are not directly assessed (though criticality and other scaling of displays might be used to address this issue.

Source provides some empirical data for the two experimental evaluations to suggest a validation of the earlier Display-Control Analyses.

N/A excepting to the On-Track study where apparently only ideal daylight driving conditions were experienced.

Studies illustrated dangers of using Time alone as a sole criteria for workload. Time and Errors were both impacted by the different experimental conditions in both evaluation studies (with uncontrolled time-error tradeoffs apparent). 


\section{Risk Taking}

Behavior:

Methodology:

Reviewer:
Risk taking behaviors are no addressed as such.

Display and control analyses were combined with limited laboratory analog and on-track studies to derive and partially validate criteria for standardized control locations in cars, trucks, and buses. Evaluation experiments contraindicate use of time as sole measure of workload.

M\&night, A. J., Shinar, D., and Hilburn, B. (1991). The visual and driving performance of monocular and binocular heavy-duty truck drivers.Accid. Anal. \& Prev, 23, 225-237.

Data Type: $\quad$ On the road and test track truck driving.

Task Listing: $\quad$ See attached table.

TaskStructure: None.

Driver: $\quad 40$ monocular and 40 binocular heavy truck drivers. Average age $=45$.

Average years of truck driving experience $=21.5$ (monocular) or 16.8

(binocular). [Difference not significant.]

Timeline Analysis: None.

Safety Criticality: Tasks in attached table are considered safety critical, especially for monocular drivers, based upon previous research cited in article.

Driver Information

Needs:

Visual performance test battery to measure static visual acuity, dynamic visual acuity, low illumination acuity, glare resistance, glare recovery, visual field, depth perception, and contrast sensitivity.

Operational

Validity:

High: Empirical results.

Driving

Conditions:

Day and night. On-street and off-street.

Criteria for

Safety Thresholds: Not discussed explicitly.

Usefulness

of Data:

No tasks performed in the highway environment showed differences between monocular and binocular drivers.

Risk Taking

Behavior:

Not discussed. 
Methodology: $\quad$ Test battery correlated with driving measures.

Reviewer: $\quad$ B. Kantowitz.

\begin{tabular}{||l|l|l||}
\hline \multicolumn{3}{|c||}{ TABLE 3.6 DRIVING PERFORMANCE MEASURES } \\
[SOURCE: McKNOGHT., SHINAR, AND HULBN, 19911.
\end{tabular}


Reid, L. D. (1981). A survey of recent driver steering behavior models suited to accident studies. Accident Analysis \& Prevention, 34, 23-40.

Data Type:

Task Listing:

Task Structure:

Driver:

Timeline Analysis:

Safety Criticality:

Driver Information
Needs:

Operational

Validity:

\section{Driving}

Conditions:

Criteria for

Safety Thresholds:

Usefulness

of Data:
Automotive driver steering models (8) published between 1975-1980 suited to accident studies.

Steering "control" aspect of driving task. (This can be thought of as a lowest part of the hierarchy of: (i) "navigation", (ii) "guidance," and (iii) "control.")

DRIVEM (Wolf \& Barrett, 1978a\&b), one of the models, breaks the steering task into three sequential components: (i) detection of critical event (scanning etc.), (ii) decision on required action, and (iii) open and/or closed-loop control.

Various populations used to develop models. Parameter variations used to characterize individual differences.

None shown but can be typically derived from model outputs.

DRIVEM characterizes 11 accident potential scenarios involving vehiclefollowing, merging, right-angle conflicts, and stationary obstacles.

Models are implicitly very specific with regard to information drivers use/need (e.g., roadway curvature).

DRIVEM and other model structures probably have evolved since this paper was written. Certainly other model types have emerged (e.g., Optimal Control). Validation of models is limited at best at the time of this report.

Conditions not specified but some environmental variations possible in some models.

DRIVEM, and other models to some extent, provide performance criteria (e.g. probability that an accident will occur under specified conditions and their variations).

Temporal safety margin and other safety-related distributions can be established using models. 
Risk Taking

Behavior:

Methodology:

Reviewer:

Walton, N. E., and Messer, C. J. (1973?). Warranting fixed roadway lighting from a consideration of driver workload. XXXXXXXX, 9-21.

Data Type:

Task Listing:

Task Structure:

Driver:

Timeline Analysis: $\quad$ Task timelines could be derived from model but are not given in paper.

Safety Criticality: Model identifies "positional" control needs as paramount for safety, but "situational" needs are certainly critical as well. These distinctions appear of limited value in terms of assessing impact of applying new technologies within

heavy vehicles.

Driver Information

Needs:

\section{Operational \\ Validity:}

Not formally discussed. However, many risk taking behavior effects can be assessed using models (e.g, as a simple parameter variation, "speeding effects" can be assessed directly).

DRIVEM, and other models to some extent, incorporate implicit task analyses. These analyses are expressed in their model structures.
Automotive driver workload (TR/TA) model for various roadway and lighting conditions for use in determining warranted lighting.

Driving task is divided into a hierarchy of levels:

(i) "Positional" informational search and control,

(ii) "Situational" information search, and

(iii) "Navigational" information search. .

Drivers service their information needs in a cyclic order with Positional requirements meet before Situational, and Situational before Navigational (as time permits).

Driver model parameters based upon: (i) various mean values observed in various field conditions, and (ii) values extracted from previously established values.

Model is explicit with respect to nature of information types and cues vis-a-vis positional, situational, and navigational information needs.

No validation in this report beyond limited parameter selection based upon small studies and previous models, but may have evolved since paper was written. 
Driving

Conditions:

Criteria for

Safety Thresholds:

Usefulness

of Data:

Risk Taking

Behavior:

Methodology:

Reviewer:
Aimed at a variety of roadway and lighting conditions.

Model indicates a workload safety threshold that is isomorphic with the Tie Required (TR) being less than the Time Available (TA): TR/TA $\leq 1$.

TR/TA approach has historically proven valuable in military and commercial aircraft settings. Bittner and Morrissey (1990) note that: (i) the historical criteria is TR/TA $<.7$, and (ii) TR variance and other important aspects are not addressed by the criteria.

Not explicitly modeled (though vehicle speed could be used as a surrogate).

The conceptual positional-situational-navigational hierarchy appears a reasonable starting point for analysis of a wide range of situations. TR/TA and extensions are likely to be value for analysis of device task interactions (cf., Bittner \& Morrissey, 1990).

Mourant, R. R., and Rockwell, T. H, (1971). Visual scan patterns of novice and experienced drivers. In Psvcholoeical Aspects of Driver Behavior, Volume 2 (Applied Research) (section II.2.A, pp. 1-19). Washington, D.C.: Department of Transportation NHTSA.

Data Type:

Analysis of novice and experienced car driver eye-movements during five onroad tasks with three levels of training.

Task Listing: $\quad$ Driving tasks were characterized grossly as:
(i) "Stop Sign 1",
(iii) "Traffic Light,"
(iv) "Approach Left Turn," and
(v) "Approach Right Turn."

Task Structure: None.

Driver: $\quad$ Novice drivers were 16-17 years old with 20/20 vision and less than 15 min. behind wheel and experienced were $21-43$ years with at least $8 \mathrm{~K}$ miles per year over last 5 years.

Timeline Analysis: N/A.

Safety Criticality: Source does not indicate what tasks are critical from a safety standpoint. 
Driver Information

Needs:

Operational

Validity:

Driving

Conditions:

Criteria for

Safety Thresholds: None explicated.

Usefulness

of Data:

Risk Taking

Behavior:

Methodology:

Reviewer:

Summary

Burger, Smith, and Ziedman (1989) indicated in their report that assessments of truck driver workload are nonexistent and, indeed, very little is known about (car) driver load and spare capacity. The references reviewed in this section provide a preview of the diverse methods and outcomes that exist. In particular, however, it should be noted that estimates of driver loads, by driving task and driving conditions, simply are not available.

Driver workload assessment poses a conundrum. In order to develop workload protocols, one must have at least some idea of what driver workload is but one can't know what the workload is without some workload protocol (or other estimates). Workload information is useful in selecting and developing methods that measure the 'right' thing just as duration of driving tasks is important to know because this suggests about how fast or slow the workload measure must be to capture the workload effect. This is akin to knowing that, for example, when a doctor needs to know patient temperature under given circumstances a thermometer rather than a stethoscope is needed. (This does not denigrate the usefulness of stethoscopes, only their appropriateness for this particular case).

The end product of this project will be a workload assessment protocol which is suitable for measuring the driver workload impact of introducing high technology in-cab devices. To help us get to this goal, preliminary SME subjective estimates of driver loads and limited observational data probably hold the best hope for us to overcome the conundrum introduced above. There is a risk that 
such estimates will be too coarse or perhaps will mislead, but at this point in the project there seem to be few alternatives. The course of this project should allow us to refine our methods over time and the task analysis information we seek now should allow us to pick very good, if not the best, paths to workload protocol development. 


\subsection{SAFETYRELEVANTCRITERIA}

\section{$\underline{\text { Introduction }}$}

One of the goals of Heavy Vehicle (HV) driver workload evaluation is to relate, if possible, the levels and types of workload to safety, particularly accident rates. This is an ambitious goal, but one that if achieved could be extremely valuable. Under ideal circumstances, an analyst would be able to assess incremental workload created by the introduction of extra equipment or a system into the vehicle cab. The analyst could use a procedure that related the increase in workload to the predicted increase in accident rate either for the vehicle fleet, or say, per 100 million vehicle miles.

Of course, no matter what procedure is developed, it will be criticized. There are limits to the accuracy with which such predictions can be made. Nevertheless, even though such predictions may be somewhat in error, they would go a long way toward specifying acceptable levels of workload for HV drivers.

We have uncovered several potential forms of "safety-relevant criteria," that is, methodologies that may be capable of predicting accident rates based on workload level or incremental workload increases. None of these is well-developed at the present time. They do however show potential for predicting accident rates.

\section{$\underline{\text { Perel's Philosonhy }}$}

Perel (NHTSA, 1976) examined accident rates using a North Carolina data base. He attempted to relate these accident rates to in-vehicle incompatibility problems by examining reported causes. He found that it was possible to determine the proportion of all accidents caused by specific in-vehicle tasks. For example, he determined the proportion reported to have been caused by lack of attention to driving while tuning the in-vehicle radio.

Perel's goal was to determine which in-vehicle problems were inducing higher accident rates. He could then study such problems in greater detail to determine why they were causing accidents. This approach, though subject to reporting inaccuracies (as Perel has already indicated) is nevertheless capable of predicting accident rates based on "diversion of driver resources" or workload.

Assume that we can get moderately accurate accident rate data for, say, ten or more in-vehicle tasks that make different demands on driver resources, particularly visual resources. Then, if the accident rate increases as a function of visual resource usage, we can perform regression to fit the best line or curve to the data. It then becomes possible to predict the accident rate based on a visual resource usage parameter. Multiple regression could be used if more than one driver resource is to be included. This is a powerful approach, and, though subject to inaccuracies, probably represents the best available and most feasible approach to development of safety-relevant criteria. 


\section{Visual Resource Parameters Approach}

An approach closely related to Perel's approach is to directly examine visual parameters, under the assumption that there is a limit (whether soft or hard) to which these may be diverted from driving. The idea is that only a limited incursion into the visual resource is tolerable (Wierwille, 1992; Rockwell, 1988).

The specific visual measures of interest when considering visual load of in-cab devices are usually the following:

mean single glance time

mean number of glances

total glance time (sums of individual glance times) and

mean (interstitial) roadway glance time.

Mean single glance time is considered to be a measure of difficulty in extracting information. Such glance times ordinarily range between 0.6 and 1.6 seconds, with longer values associated with difficulty of information extraction. Number of glances is a measure of number of steps or complexity of procedure of performing the in-cab task. Number of glances ranges from about 1 for simple check reading to about 7 for difficult navigation tasks. Total glance time is a global assessment of the visual demand of an in-cab task and thus represents the "taxing" effect such a task has on the driver's visual resource. Finally, mean roadway glance time may be assessed in some cases and is a measure of "road load" of the driving task. For example, when faced with a potential conflict in the forward scene, glance time to the roadway increases markedly (Wierwille, Hulse, Fischer, and Dingus, 1988).

Some researchers have attempted to place limits on mean single glance time and mean number of glances (Wierwille, 1992). For example, Zwalen, Adams, and DeBald (1988) submit that single glance times should not exceed about one second and number of glances should not exceed 3 . Wierwille has suggested "that mean single glance lengths of 1.25 seconds should be considered acceptable, although values shorter than this are preferred. This later duration is at the conservative end of the average glance durations for car radio operation [ 1.27 to 1.42 seconds] reported by Rockwell(1988). In terms of number of glances, a limit of about 6 is probably acceptable, particularly when mean single glance times do not exceed 1.25 seconds.

These estimates are based on the idea that small incursions into the visual resource are acceptable and that large ones are not. The values suggested above are within the range derived from data on visual allocation to commonly used in-cab systems [HVAC, radio, etc.]. It is assumed that such subsystems interactions are reasonably safe, an assumption which is open to challenge by Perel's logic, among others. If a more scientific basis can be found or developed for specifying bounds on visual parameters, then safety relevant criteria follow. It should be possible to show that the safety-relevant aspects of driving are compromised when visual incursions exceed certain thresholds for a given set of conditions. For example, Antin, Dingus, Hulse, \& Wierwille (1986) found a strong relationship between glance frequency to the in-cab display and percent trials where lane crossings occurred but found no reliable relationship between glance duration and percent trials where lane crossings occurred. 
This is a promising approach and is useful in performing side-by-side comparisons of in-cab devices. Such devices can be rank ordered in terms of their visual demands. The main problem that remains is determining a scientific rationale for setting threshold values.

\section{$\underline{\text { Harm's Concept }}$}

In a recent study of driver cognitive load performed in Denmark, Harms (1986), used an auditory second task. The task involved responding to rapidly presented arithmetic problems. Harms showed that reaction times were longer when driving speeds were lower. These lower speeds occurred in villages where cognitive load for drivers was believed to be higher. Thus, Harms concluded that the secondary task could successfully be used to assess driver cognitive load.

In addition, she examined accident rates at various 100 meter stretches of roadway and compared them to cognitive load. She found a direct relationship between the two, although no correlation coefficient or other measure of agreement was presented.

The Harms approach suggests that cognitive load may be directly linked to accident rate, which in turn suggests the possibility of developing safety-relevant criteria based on the concept. There are some difficulties associated with Harms' concept. Hicks and Wierwille (1979) showed that secondary tasks of digit shadowing and of occlusion were not sensitive to variations in handling difficulty created by random crosswinds. This suggests that only certain types of driver load may be assessed by certain secondary tasks. That is, secondary tasks must be selected which tap into the same driver resources as those loaded by primary task demands or else a finding of "no effect" is to be expected. Furthermore, the Harms study may not be generalizable to the U.S. because of large differences in roadway design between urban areas of Europe and the U.S. Validity of the results obtained by Harms needs to be determined by replicating the experiment in other areas using other experimenters.

Finally, there is the problem of intrusion. The secondary task in Harms' research was presented auditorily using earphones. It would thus likely be intrusive in any in-vehicle task having auditory or verbal components. Furthermore, it is difficult to envision use of an additional secondary task when the driver is attempting to perform an in-vehicle task while driving. Since the in-vehicle task is also a secondary task, the two secondary tasks would have to compete with one another. While embedding is possible, there are no research results for such an approach. In spite of these difficulties, the concept of having a probe task, the results of which are known to predict accident rate, is certainly an appealing one. A key element to the success of probe tasks for predicting safety is a thorough understanding of what exactly it is they are measuring. Until some taxonomic basis is used to characterize such tasks, this key element will prove elusive.

\section{Time-to-Line Crossing (TLC) Concept}

Godthelp and his colleagues (Godthelp, Milgram, and Blaauw, 1984) have developed a so-called TLC model to determine when drivers must obtain a new visual sample of the roadway. The concept involved in this work is similar to a much older one developed by Senders, Kristofferson, Levison, Dietrich, and Ward (1967). They demonstrated that if the driver's forward view is occluded, there is an uncertainty buildup with time. The driver then needs another sample, or will immediately slow the vehicle. 
TLC (time-to-line crossing) is much the same, but hypothesizes that the uncertainty is directly related to the amount of time it would take for the vehicle to cross either of the two lines marking the driving lane, assuming the steering wheel is held constant. This time represents a "safety margin" in that, when there is no traffic, the vehicle is safe as long as it remains in its lane.

Since TLC can be calculated assuming transverse disturbances are small, it represents a form of safety relevant criterion. To see how this could be applied, consider that between visual samples to the roadway a driver attempts to perform in-vehicle tasks. As long as the driver returns glance to the forward view in a time less than TLC, there should be no problem in controlling the vehicle. This is tantamount to specifying the maximum length of time the eyes can be off the forward view.

The TLC concept has shortcomings. It does not account for traffic, emergencies, or crosswinds and transverse roadway disturbances. Furthermore, HVs are subject to greater variation in their trajectories when steering is fixed than are automobiles (HVs require more careful steering control). Thus, uncorrected TLC-derived values would permit much longer glances into the heavy vehicle than would ordinarily be considered safe. Perhaps the concept could be extended to include these other issues. However, we might as well go back then to Senders et al's original concept, which is based on occlusion measurement and information theory.

\section{Manual Loading Approach}

It is possible to develop safety-relevant criteria for manual loading in a manner similar to that described earlier as the Visual Resource Parameters Approach. The approach is based upon measures and cutoff values, as previously described.

For manual loading the most important measure appears to be "hand-off-wheel time." To obtain this measure, drivers are instructed to begin the task with both hands on the wheel and to return the used hand to the wheel on completion of the task. Hand-off-wheel time then becomes a measure of total manual demand of an in-cab task. Additional measures having some value are hand transition time in each direction (that is to the control from the wheel and vice versa) and actuation time (the time that the hand is on the control).

Since vehicle control is probably not as precise with only one hand on the wheel, it may be possible to relate manual demands to safety. Furthermore, there may be an upper limit or threshold value which, say, hand-off-wheel time should not exceed. There are at present no data on which to specify such a threshold, thus limiting the potential application of such an approach. Furthermore, it is at least theoretically possible to drive for very long stretches with only one hand on the wheel (or no hands if the roadway is level). Indeed, Viano, Patel, \& Ciccone (1989) analyzed arm position from films of 1,890 vehicles and reported that drivers used their armrest 34.4 percent time on the open road and the driver's left hand was on the steering wheel only 69.6 percent of the time when stopping at intersections. However, heavy vehicles may be less tolerant of one-hand operation and the configuration of American trucks implies many add-on devices will be positioned to the right of the driver. Thus, drivers must use their [normally dominant] right hand for device manipulation while attempting to steer with the non-dominant hand. 
Biomechanical Interference Approach

Heavy vehicle drivers depend heavily on their side view mirrors for situation awareness while driving. Due to the presence of the trailer, rear view mirrors are irrelevant and head turning is often of little use (especially in cabs with sleepers). Therefore, another safety-relevant criterion may be the "biomechanical interference" induced by an in-cab device. This may be defined as the extent to which the device, by its position and design, prompt the driver to lean out of the neutral driving posture. This effect would, in principle, change the viewing angles to the side view mirrors and thus compromise safety by effectively occluding vision to the road scene in back and to the side of the driver. This effect would also potentially affect control of the vehicle by changing foot angle to the accelerator, brake, and clutch pedals, by perhaps increasing steering errors, and so on. Given that some in-cab devices may be located a distance from the driver, this approach merits further consideration.

As a measurement problem, biomechanical interference potential might be quantified as follows. Reach envelopes (relative to the Seat Reference Point (SRP)) would be used to analytically determine the degree to which a driver of a given size would have to leave the neutral sitting posture to manipulate the device. It may be possible to describe a range of reach envelopes (e.g., 5\%-ile female, $5 \%$-ile males) beyond which device placement is unacceptable. Studies could be conducted to determine the range of reaches beyond which visual angles to the side view mirrors are unacceptably degraded or steering and pedal activation is compromised. Given significant variation in mirror size, shape, orientation, tractor trailer profile, and cab geometry, this could be challenging work. However, such an approach would lend itself well to a computerized prediction model.

\section{$\underline{\text { Other Resource Loading Approaches }}$}

Theoretically, it is possible to assess any resource the driver may use to perform an in-cab task. We have already discussed visual and manual resources in detail. There remain the cognitive, verbal, auditory, and pedal resources. If we assume for the moment that various resources do not interact, then individual limits could conceivably be specified for measures associated with each resource. On the other hand, if the resources do interact, then some combined limit procedure must be used which allows some trading of one resource for another.

The problem with the approach is that theoretically it is possible to use, say, the verbal resource almost continuously without creating a known, definitive hazard in driving. Thus, specification of limits may not be scientifically justifiable and therefore not defendable. As Wierwille (1992) has pointed out, it may well be desirable to reduce visual load by using other resources. It is precisely because these other resources are not as safety-relevant that they can be drawn upon.

\section{$\underline{\text { Summary }}$}

Deriving the safety relevant criteria for candidate measures of driver workload will prove to be a difficult and scientifically heated endeavor. For any measurement technique, someone will question its validity and offer a counterexample or experimental contraindication. Are these to be taken seriously or are they to be written off as some eccentric demonstration concocted to prove that, theoretically, 'it all depends'? For any recommended range of 'acceptable' or 'unacceptable' 
measures, someone could question their validity and generalizability. Tradeoffs will have to be made, e.g., how acceptable is it to incur a slightly greater glance frequency for richer emergency traffic information which can keep the driver from dashing headlong into harm's way? From a statistical population standpoint, where should criteria reside, e.g., is it defensible to only protect $95 \%$ of all drivers? From a measurement standpoint, will the state-of-the-art allow for such precision in any event? It may well be that the most prudent goal is to identify candidate criteria or measures with which to conduct relative comparisons though this still begs a host of issues.

Perel's philosophy is, conceptually, an ideal approach to establish safety relevance. If only there were sufficient data from accident reports to identify in-cab transactions that precipitated an accident. If only there were a human performance taxonomy with which to characterize various in-cab devices using a common vocabulary. If only there were known relations between that vocabulary and driver loads or resources. The models thus derived would go a long way toward addressing some of the issues mentioned in the preceding paragraphs. A great deal of work, including more refined accident reporting, is needed to make this approach a reality. Conversely, any in-cab device which improves driver performance can only be inferred to reduce accidents since accidents are rare events based on any exposure metric.

Given the primacy of vision to safe driving, the relevance of visual allocation measures is apparent. Glance duration and frequency information has high face validity, has demonstrated relationships to driving performance, and so will undoubtedly be a significant part of any useful workload assessment protocol. It is not a panacea, however. The suggestion to use existing visual allocation data for conventional in-cab systems to derive safety relevant criteria is problematic to the extent that conventional in-cab systems (e.g., complex stereo systems) may sometimes be dangerous to use (though this might be partialled out by some means). The visual allocation (distinct from eye movement measurement per se) approach will likely be useless for in-cab devices which have, say, only auditory or kinesthetic-tactile displays. Wierwille (1991, submitted for publication) has made the point that more refined eye movement measurements may be useful even in these circumstances because high cognitive loading can lead to perceptual narrowing, manifested by a temporary suppression of visual scanning needed for hazard detection. Nonetheless, the level of complexity for data collection and analysis increases sharply.

Harm's concept of relating secondary (or, indeed, any) workload measurements to driving safety is troubling for the reasons mentioned earlier. The significance of the result rests on the fact that variations in the secondary task varied with other observable indicators of workload (e.g., road type and location in villages vs. open highways). Thus, one is prompted to ask what value the secondary task measure provided that these other observables could not provide more directly? Similarly, correlating variations in secondary task performance with accident rates per unit length of road is fraught with problems, e.g., hazard exposure differences and assuming causation from a correlation. (These problems, it must be said, plague all methods to some extent). The challenge (and the benefit) of the secondary task approach lies in elucidating the locus of such effects and providing information which cannot be gathered any other way. If visual allocation is judged to assess primarily structural interference (you can't look foveally in two directions at once), then secondary tasks may complement workload assessment by providing insights into central processing: limitations. However, it remains to be demonstrated that visual allocation, in heavy vehicle driving, does not sufficiently gauge, say, attentional deployment, thus making additional measures both useful and necessary. 
The TLC measure has appeal as a primary driver workload metric for lane keeping. Others will also be sought out in Task 4 work (e.g., Steering Wheel Actuation Rate, pedal reversals, etc.). These quality of driving measures should support a more direct relationship to safety on the road, but much of this relationship may have to be developed by logical argument. For example, TIC is attractive because it seems self-evident that crossing the centerline or the fog line degrades safety and reduces the drivers' options. One vexing aspect of driver performance, however, is that 'quality of driving' is broadly defined. Drivers do not necessarily try to maintain zero lane deviation, for instance, and so increased lateral tracking error may not be nearly so important as, say, range of lateral excursions.

Manual loading and biomechanical interference have intuitive appeal and so should be considered further. Given that truck drivers sometimes drive with only one hand, the impact of in-cab device manipulation on safety is called into question. Perhaps it should be, unless those manipulations are for, say, visually guided movements. The taxonomy developed by Wierwille and presented in Section 1.0 (Table 1.1) becomes increasingly important for determining what manual loadings are safety critical and what loadings are not. Similarly, the degree of biomechanical interference which is allowable is currently undetermined.

Oftentimes, human factors professionals do not have cutoff values, thresholds, and firm guidelines upon which to assess systems or influence their design. In these cases we strive to minimize (e.g., glance frequency, resource competition), or maximize (range of adjustability) in a relative sense. While opportunities for more absolute criteria do exist, safety relevant criteria may need to be addressed in a relative way for the application of the to-be-developed workload assessment protocol. 


\section{$\underline{\text { References }}$}

Antin, J.F., Dingus, T.A., Hulse, M.C., and Wierwille, W.W. (1986, November). Human factors test and evaluation of an automobile moving-map navigation svstem. Part I: Attentionaldemand requirements: Part II: Methodology issues- Effectiveness. efficiency and strategy. Blacksburg, VA, UPI and SU.

Godthelp, H., Milgram, P. and Blaauw, G. J. (1984). The development of a time-related measure to describe driver strategy. Human Factors, 26(3), 257-268.

Harms, L. (1986). Driver's attentional responses to environmental variations: a dual-task real traffic study. In A. G. Gale (Ed.), Vision in Vehicles (pp. 131 - 138). Amsterdam: North-Holland, pp. $131-138$.

Hicks, T. G. and Wierwille, W. W. (1979). Comparison of five mental workload assessment procedures in a moving-base driving simulator. Human Factors, 21(2), 129-143.

National Highway Traffic Safety Administration (NHTSA) (1976). Analyzing the role of driver/vehicle incompatibilities in accident causation using police reports (Report No. DOT-HS-801858). Washington, DC: U.S. Department of Transportation. (Author, M. Perel; March).

Rockwell, T.H. (1988). Spare visual capacity in driving - revisited: New empirical results for an old idea. In A.G. Gale et al. (Eds.), Vision in vehicles II (pp. 317-324). Amsterdam: Elsevier.

Senders, J. W., Kristofferson, A, B., Levison, W. H., Dietrich, C. W., and Ward, J. L. (1967). The attentional demand of automobile driving. Highway Research Record, 195, pp. 15-32.

Viano, D., Patel, M., and Ciccone, M. (1989). Patterns of Arm Position During Normal Driving. Human Factors, 31(6), 715-720.

Wierwille, W. W. (1992). Demands on driver resources associated with introducing advanced technology into the vehicle. Paper submitted for possible publication in Transportation Research. (Currently under review.)

Wierwille, W. W., Hulse, M. C., Fischer, T. J., and Dingus, T. A. (1988). Strategic use of visual resources by the driver while navigating with an in-car navigator display system, XXII FISITA Congress Technical Papers; Automotive Systems Technology: The Future, Vol. II, SAE P-211. Warrendale, PA: Society of Automotive Engineers (September) 2.661-2.675, (Paper No. 885180).

Zwahlen, H. T., Adams, C. C., Jr., and DeBald, D. P. (1988). Safety aspects of CRT touch panel controls in automobiles. In A. G. Gale (Ed.) Vision in vehicles II (pp. 335 - 344). Amsterdam: North-Holland Press. 


\subsection{RISK TAKING AND WORKLOAD}

\section{Introduction}

There is little doubt that some degree of risk is associated with virtually all important activities engaged in by operators of motor vehicles. This is certainly true of heavy vehicle drivers. It is not surprising then that as models of driver behavior and theories of accident involvement have evolved, the concept of risk has become a prominent component in virtually all these models (c. f., McKenna, 1988; van der Molen \& Botticher, 1988). In fact, a special issue of the journal Ergonomics (Volume 31,1988 ) was devoted to the topic of "Risky Decision Making in Transport Operation." However, if one examines the twenty-one papers presented in this issue of the journal a number of research issues relevant to our workload assessment study become clear. This section will summarize these points.

\section{The Role of Risk in Models of Driving Behavior}

In the most prominent models of driving behavior (Wilde, 1982a, 1982b), there is a distinction between two kinds of risk. First there is objective risk which is quantified in a given domain on the basis of the assimilation of stable relative frequency data over an extensive number of observable periods (e.g., annual fatalities, probability of death per hour of driving time, etc.). The second type of risk, subjective risk, refers to an individual's perception of the degree of risk associated with a particular behavior. The relationship between these two variables forms the basis of an important component of models of driving behavior.

For example, in Wilde's (1982a, 1982b) Risk Homeostasis Theory, the model assumes that an individual has a desired level of risk that he or she will maintain. This level need not be and for most individuals will not be some zero level. Such an assumption has been used to form the basis of psychological models of general risk-taking behavior (e.g., Coombs' Portfolio Theory, 1975), and appears to have significant empirical support. In his model, Wilde (1982a) proposes that decision making in driving behavior will be guided by the discrepancy between the perceived level of risk and the desired level. "If the level of subjective risk is higher or lower than the level of risk desired, the individual will take action in an attempt to eliminate this discrepancy (p. 212)."

Other models include the concept of subjective risk as well. Naatanen and Summala (1976), for example, have also developed a model of driver decision making that has perceived risk as a central component. In their model there exists within the individual a "Subjective Risk Monitor", that has a unique threshold value. When this threshold is exceeded (i.e., when perceived risk is higher), the driver's subsequent behavior is predicted to be altered.

\section{Gaps in the Data Base}

An important conclusion to be drawn from this research is that although there are compelling theoretical arguments for the inclusion of perceived risk as a significant component of models of driving behavior, there are at least two major gaps in the research as it relates to this heavy vehicle assessment study. First, there are very few empirical studies of quality that have attempted to test hypotheses concerning the roles of perceived risk and predisposition toward risk-taking in these models. Many attempts to support or reject these models are based on theoretical exercises (e. g., 
van der Molen \& Botticher, 1988) with questionable validity. Studies with strong empirical work are rare.

An interesting exception was presented in the 1988 special issue of Ergonomics A study by Riemersma (1988) attempted to examine how drivers internally represent features and functions of traffic system components. Subjects in the study were shown slides of 28 sections of road and made judgments about them on 22 different constructs, The data were analyzed via multidimensional scaling and clustering techniques in an attempt to examine how the drivers cognitively represented and categorized the conditions and how these psychological representations matched with objective characteristics. A similar approach could be used successfully to assess truck drivers' psychological representations of events that have low to high perceived risk components. Multidimensional scaling techniques could be used to analyze the data.

Secondly, no empirical work appears to have been reported on risky decision making and driving behavior of heavy vehicle operators. This lack of research has several practical implications. First, it suggests that a basis for choosing measures to study decision making in heavy vehicle operators will have to be developed. There is virtually no established work that would support any particular measures as having anything other than face validity at this point. Secondly, there does not appear to exist any empirical evidence to suggest that what data is available from studies of automobile driving behavior generalize to heavy vehicle operation. Such generalizations will need to be established.

\section{Workload and Risk-Taking Behavior}

There appears to be only one recent paper that begins to address the relationship between mental workload and risk-taking in driving behavior. In that paper, Hoyos (1988) attempts to show the value of a mental-load approach to the modelling of traffic behavior. Although, he argues for an approach that tries to "understand the cognition and recognition of hazards as the comprehension and processing of hazard indicators" (p. 580), there is very little that is new in this paper with respect to establishing useful valid measures. Basically, two approaches were suggested. Extensive interviews were used to identify the hazard indicators that individuals recognized, and subjective rating scales were used to assess perceived stress (workload) on test drivers under different traffic conditions.

\section{$\underline{\text { Summarv }}$}

In sum, based on the sparse literature covering the relationship between workload and risk taking behavior in heavy vehicle operators, three points seems clear.

1. The concept of risk is established only at a theoretical level in models of driving behavior. From a psychological standpoint, the work thus far is very weak and disjointed. What little empirical work there is not tied well to theory.

2. However, only a very small empirical data base exists for studying risk taking in automobile driving behavior (virtually all of it done in Europe), and no data base exists for heavy vehicle operators. 
3. The literature suggests that there are no established measures for assessing risk-taking in driving behavior. Such measures will need to be developed.

4. The relationship between risk taking and workload is not defined in the literature reviewed. Thus, there is no published justification in the literature reviewed to expect risk taking to raise or lower in the face of in-cab device induced workload (or vice versa).

\section{References}

Coombs, C. H. (1975). Portfolio theory and the measurement of risk. In M. Kaplan \& S. Schwartz (Eds.), Human judgment and decision processes. New York: Academic Press.

Hoyos, C. G. Mental load and risk in traffic behavior. (1988). Ergonomics, 31, 469-484.

Huguenin, R. D. The concept of risk and behavior models in traffic psychology. (1988). Ergonomics, 31, 557-569.

McKenna, F. P. (1988). What role should the concept of risk play in theories of accident involvement? Ergonomics, 31, 469-484.

Naatanen, R., and Summala, H. (1976). Road user behavior and traffic accidents. Amsterdam: North Holland.

Riemersma, J. B. J. (1988). An empirical study of subjective road categorization. Ergonomics, 31, 621-630.

van der Molen, H. H., and Botticher, M. T. (1988). A hierarchical risk model for traffic participants. Ergonomics, 31, 537-555.

Wilde, G. J. S. (1982a). The theory of risk homeostasis: Implications for safety and health. Risk Analysis, 2, 209-225.

Wilde, G. J. S. (1982b). Critical issues in risk homeostasis theory. Risk Analysis, 2, 249-258. 


\subsection{DRIVING TASK ANALYSIS DATA COLLECTION PROTOCOLS}

In this section, a variety of methods which have been used for driver task analysis data collection are described briefly. Their relevance and potential for the current effort are described. These methods span the range of subjective, observational, and behavioral techniques. Their diversity reflects the complexity of characterizing driver behaviors and the need for a multifaceted approach; no single technique is likely to be satisfactory. The use of multiple techniques may provide both unique information and converging operations for areas of overlap in analysis of the job of driving heavy vehicles.

\section{Activity Analysis/Work Sampling}

This is an established human factors method useful for determining the proportion of time spent in different activities which comprise a job. In the case of automotive applications, Rabideau and Young (1974) used activity sampling based on 20 second segments, randomly selected. A total of 60 such segments were used per 8 hours of driving and ride-along data collectors used a pre-established activity sampling sheet for data collection purposes (See Table 6.1). Frequency of occurrence ranged from 1 to 60 in terms of number of segments during which an activity took place. Other conditions, e.g., weather, traffic density, nature of the road segment, were noted but not included in tabled results,

In order to use this method, a variety of steps must be taken (Meister, 1985). A sampling strategy must be determined (e.g., random, fixed interval, ratio delay, event-based). A sample duration must be decided upon. Data collection forms with the desired behaviors (defined at the desired level of description) must be prepared. The driving conditions and tasks to be sampled must be decided upon. A desired sample size to be collected must be chosen (e.g., 60, 100, 500). Finally the data collector must receive some training in observation and use of the driver behavior taxonomy, the recording of multiple concurrent activities, and so on.

Activity sampling is useful for determining the frequency with which observable driver behaviors occur for specified driving conditions. It is a relatively simple method to apply and data reduction and analysis is reasonably straightforward. However, activity sampling cannot be used for unobservable (e.g., cognitive) activities. It depends heavily on the observer's performance and his ability to categorize behaviors, observe multiple behaviors occurring concurrently, and describe the driving conditions under which the behaviors arose. It nonetheless holds promise for use in this project to identify the frequency of events.

\section{Personal Interviews}

Interviews with subject matter experts have also been used extensively. Again, referring to Rabideau and Young (1974), interviews were conducted with long haul truck drivers to identify their attitudes and subjective assessments of selected aspects of truck driving. (See Table 6.2). While these authors do not provide sufficient detail on the nature of their interviews, they did use a combination of openand close-ended questioning to address the topics indicated. Structured interviews can also use 


\section{TABLE 6.1. ACTIVITY SAMPLING SHEET USED FOR LONGHAUL DRIVER TASK}

ANALYSIS. (SOURCE: RABIDEAU AND YOUNG, 1974).

(Segment Duration - 20 Secs.)

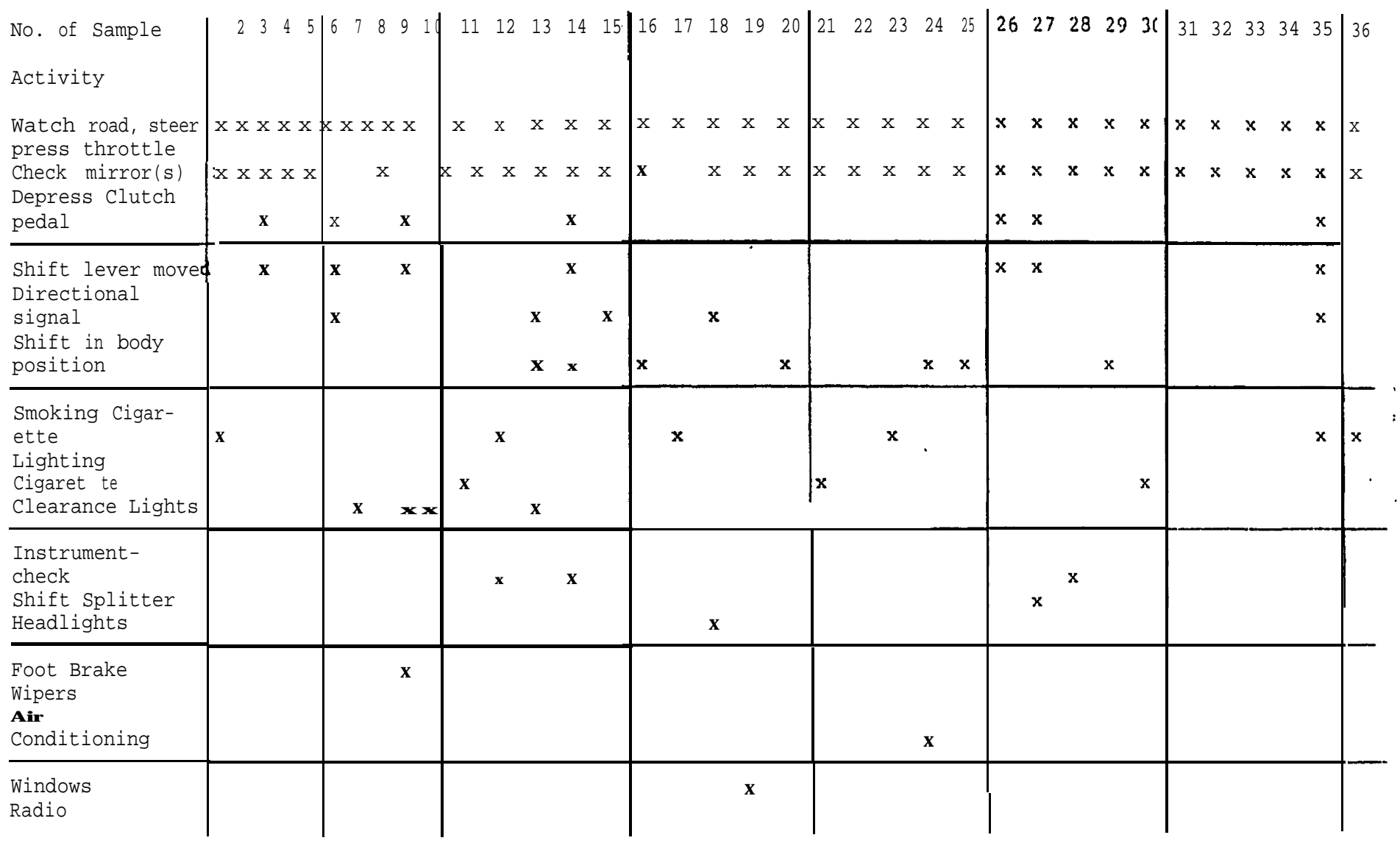


TABLE 6.2. INTERVIEW DATA FROM LONG-HAUL DRIVER TASK ANALYSIS (SOURCE: RAFHDEAU AND YOUNG, 1974).

\begin{tabular}{|c|c|c|c|}
\hline Topic & Positive Comments & Negative Comments & Inferences \\
\hline General Safe Driving & $\begin{array}{l}\text { Attention to driving plus sensible decisions } \\
\text { ranked highest }\end{array}$ & $\begin{array}{l}\text { Skill in handling rig ranked } \\
\text { as low requirement }\end{array}$ & $\begin{array}{l}\text { Information processing is more } \\
\text { important than effector output } \\
\text { skills }\end{array}$ \\
\hline $\begin{array}{l}\text { Risk Avoidance in Unfamiliar } \\
\text { Situations }\end{array}$ & $\begin{array}{l}\text { Caution and planning ahead for worst case } \\
\text { possibilities }\end{array}$ & $\begin{array}{l}\text { Quick thinking and use of } \\
\text { avoidance or escape } \\
\text { techniques downrated }\end{array}$ & $\begin{array}{l}\text { Maneuverabilityandbraking } \\
\text { abilities of trucks is poor. Use of } \\
\text { caution is easier on driver over } \\
\text { many hours than being tensed } \\
\text { and ready for instant accident } \\
\text { avoidance. }\end{array}$ \\
\hline Other Vehicle Behaviour & & $\begin{array}{l}\text { Lack of signaling of } \\
\text { intentions and general lack } \\
\text { of predictable bchaviour }\end{array}$ & $\begin{array}{l}\text { Contingency planning by truck } \\
\text { driver is difficult if not useless. }\end{array}$ \\
\hline Day vs. Night Driving & $\begin{array}{l}\text { Day driving preferred by } 40 \% \text { (approx.) } \\
\text { because of ease of staying awake, better } \\
\text { scenery and greater number of interpersonal } \\
\text { contacts. }\end{array}$ & $\begin{array}{l}\text { Night driving preferred by } \\
\text { approx. } 50 \% \text { because of } \\
\text { reduced traffic density, and } \\
\text { ease of other vehicle } \\
\text { location using headlamp } \\
\text { glare or aura. }\end{array}$ & $\begin{array}{l}\text { Day driving reduces problems of } \\
\text { alertness but night driving allows } \\
\text { for easier sensing of certain } \\
\text { critical stimuli. }\end{array}$ \\
\hline Visual Search Techniques & * & * & $\begin{array}{l}\text { Visual search patterns are } \\
\text { relatively automatic or non- } \\
\text { conscious, but quite complex and } \\
\text { rigorous. Likely require much } \\
\text { practice to develop to highly } \\
\text { effective stage. }\end{array}$ \\
\hline Critical IncidentCauses & & $\begin{array}{l}\text { Unexpected behaviour by } \\
\text { other vehicles which is often } \\
\text { very dangerous by itself. } \\
\text { Lack of attention to driving } \\
\text { task either through } \\
\text { daydreaming or actually } \\
\text { starting to fall asleep. }\end{array}$ & $\begin{array}{l}\text { Situation in which driver finds } \\
\text { himself is impossible to predict } \\
\text { except on contingency basis and } \\
\text { may be impossible to avoid. } \\
\text { Lack of attention causes } \\
\text { immediate stop or reduction of } \\
\text { information gathering and } \\
\text { processing and subsequent similar } \\
\text { changes to outputs. }\end{array}$ \\
\hline Route Pacing & & $\begin{array}{l}\text { Drivers disliked working for } \\
\text { companies which had } \\
\text { specific route schedules for } \\
\text { time to distances, etc. }\end{array}$ & $\begin{array}{l}\text { Drivers preferred Io set speeds to } \\
\text { mad and personal conditions; to } \\
\text { set objectives based on variable } \\
\text { fatigue rates etc. Different road } \\
\text { speeds allow driver to modulate } \\
\text { information processing rates to } \\
\text { suite his abilities at that time. }\end{array}$ \\
\hline Rest Stop Criteria (Timing) & $\begin{array}{l}\text { Most drivers used criteria of physical feeling } \\
\text { (discomfort) and noticeable performance } \\
\text { decrement (poor shifting) or availability of a } \\
\text { familiar pleasant stop point. On familiar } \\
\text { mutes former criteria were not used as } \\
\text { frequently as latter. }\end{array}$ & $\begin{array}{l}\text { Worst time to be on mad is } \\
\text { period from } 4-6 \text { am. Most } \\
\text { drivers try to eat through it } \\
\text { or sleep 2-4 hrs. in this } \\
\text { area. }\end{array}$ & $\begin{array}{l}\text { Drivers recognize a diurnal low } \\
\text { point in performance. Subjective } \\
\text { self evaluation of performance by } \\
\text { driver does not generally yield } \\
\text { clear, repeatable criteria for } \\
\text { taking breaks. Driver will } \\
\text { disregard fatigue symptoms to } \\
\text { drive to a desirable stop. }\end{array}$ \\
\hline
\end{tabular}

All drivers had great diffkulty in verbalizing their techniques yet all admitted it was one of the most important aspects of safe driving. General feeling was that a driver could not be too observant - he had to see everything. 
videotapes of nms or scenarios to elicit from the driver answers to such questions as the following (Hannigan and Parke-s, 1988): how do you do this?, why do you do this?, what do you do next?; what do you do at the same time? Interviews will thus form an essential element in Task 3 data collection.

\section{$\underline{\text { Commentarv Driving/Protocol Analysis }}$}

An important technique for uncovering driver cognitive operations is commentary driving or protocol analysis, an introspective technique wherein the driver 'talks aloud' about the driving task. It may be done during task execution, or afterward (perhaps with the use of a videotape of the task). Hannigan and Parkes (1988) indicate that a major cause for concern with this method is its introspective nature. It is possible that drivers' comments may omit critical elements of the driving situation or driver strategy, may include false items which are based on the driver's prescriptive (rather than descriptive) internal model of the task, or may generate irrelevant items which do not provide help in understanding the driving situation and driver responses to that situation. It is also well known that some people are not able to articulate their thought processes well and may resort to verbalizing low level behaviors (e.g., "Now I'm tuning the wheel", rather than "I'm turning the wheel because..."). Finally, tasks which are highly automated may not be articulated well (because they are beyond the driver's consciousness) and quite difficult tasks may be equally difficult to articulate.

The analysis of the verbal protocol ranges from a content analysis to uncover driver strategies, critical information requirements and the like, to a translation of the verbal content into a structured format suitable for building computer simulations (called productions) of the human. While it is expected that a content analysis would be sufficient for the current effort, this method is labor intensive to apply though it currently offers a unique insight into the cognitive processes drivers use. It is a way to identify, a task context, the information requirements of drivers, the strategies they use to deal with a particular driving situation, and the processing that they go through in a task. Therefore, it is anticipated to be useful in later tasks of this project. One variation of its use may be to present a driver with a scenario in an interview format and ask the driver to verbalize the data they would attend to, decision processes they would undertake, and responses which would be made. Such an approach may help to verify existing truck driver tasks and uncover new ones.

\section{Critical Incident Technique/Critical Decision Method}

The Critical Incident Technique (CIT) and variants such as the Critical Decision Method (CDM) require the driver to recall 'close calls', describe them, and speculate on their causes. This method obviously depends on the driver's ability to recall such details and is open to distortions of memory and motivation. While relating critical incidents, drivers may cast themselves in a more favorable light, are likely to distort the frequency or duration information about an event, and will likely ignore the more routine aspects of driving. Despite this, CIT has proven to be a robust and useful methodology in identifying safety-critical situations which can help us in this current effort to identify the driving conditions and tasks which merit close scrutiny. Such data will have to be treated as estimates to be verified through other means. 


\section{Subjective Workload Ratings}

One approach to workload estimation (which has been used in an aviation context) is to have professionals, well acquainted with heavy vehicle driving, rate the degree of loading attendant to a particular task. For example, Aldrich, Szabo and Bierbaum (1988) developed a set of anchored rating scales for various pilot workload factors (See Table 6.3). Something similar to this may be useful for the current effort. The negative aspects of this are the subjective nature of the assessment, the need for validation of the rating scales (e.g., at least through inter-judge concordance assessments), the need for reliability assessment of the scales (perhaps with intra-judge reliability coefficients), and the labor intensive nature of the data gathering (e.g., task-by-task assessments, varied by combinations of driving conditions). However, at this early stage of this project, this approach merits further consideration for use in Task 3. To anchor such scales, a variety of driving task descriptions (with driving conditions) would be presented to a group of drivers to rate on a scale of, say, 1 to 7 . Our driver Subject Matter Expert (SME) would help ensure that the task descriptions spanned the range of workload. Then anchors would be chosen by calculating mean ratings and standard deviations of ratings to pick the descriptions with minimum variance at the low, medium and high end of the scale. These minimum variance ratings would serve as scale anchors.

It is worth noting that the above rating scales are quite distinct from established subjective workload methods like the Subjective Workload Assessment Technique (SWAT), the Task Load Index (TLX), the modified Cooper-Harper scale, or the Bedford scale (see Hart \& Wickens, 1990 for a review). None of these methods provide the breakdown of driver loads into the categories of visual, manual, cognitive, pedal, auditory loads of interest to this project. Thus, additional task analytic data must be collected on these using a different set of techniques. The established subjective workload measures, however, can provide a useful overall assessment of the driver's perception of load.

\section{$\underline{\text { Visual Allocation }}$}

In Task 4 of this project, review and analysis of various workload measures applicable to driving will be made. Visual allocation measures (i.e., measures of glance duration and frequency in various incab device interactions) have been collected in numerous studies to characterize the visual load imposed by various in-cab devices on the driver, as well as variations in visual allocation as a function of driver experience, task type, and so on. Given the primacy of vision in driving, visual allocation and visual workload will be an important consideration for latter portions of the project.

\section{On-The-Road Driver Performance Monitoring}

Again, in Task 4 of this project, review and analysis will be carried out on driver performance measures (e.g., TLC, pedal reversals, steering wheel movement, etc.) which have been used to assess driver workload and the quality of driving. Further discussion of the relevance of driver performance monitoring is provided in the section on safety-relevant measures and criteria. 
TABLE 6.3. EXAMPLES OF WORKLOAD COMPONENTS SUITES (SOURCE OF ALDRICH, SZABO, \& BIERBAUM, 1989)

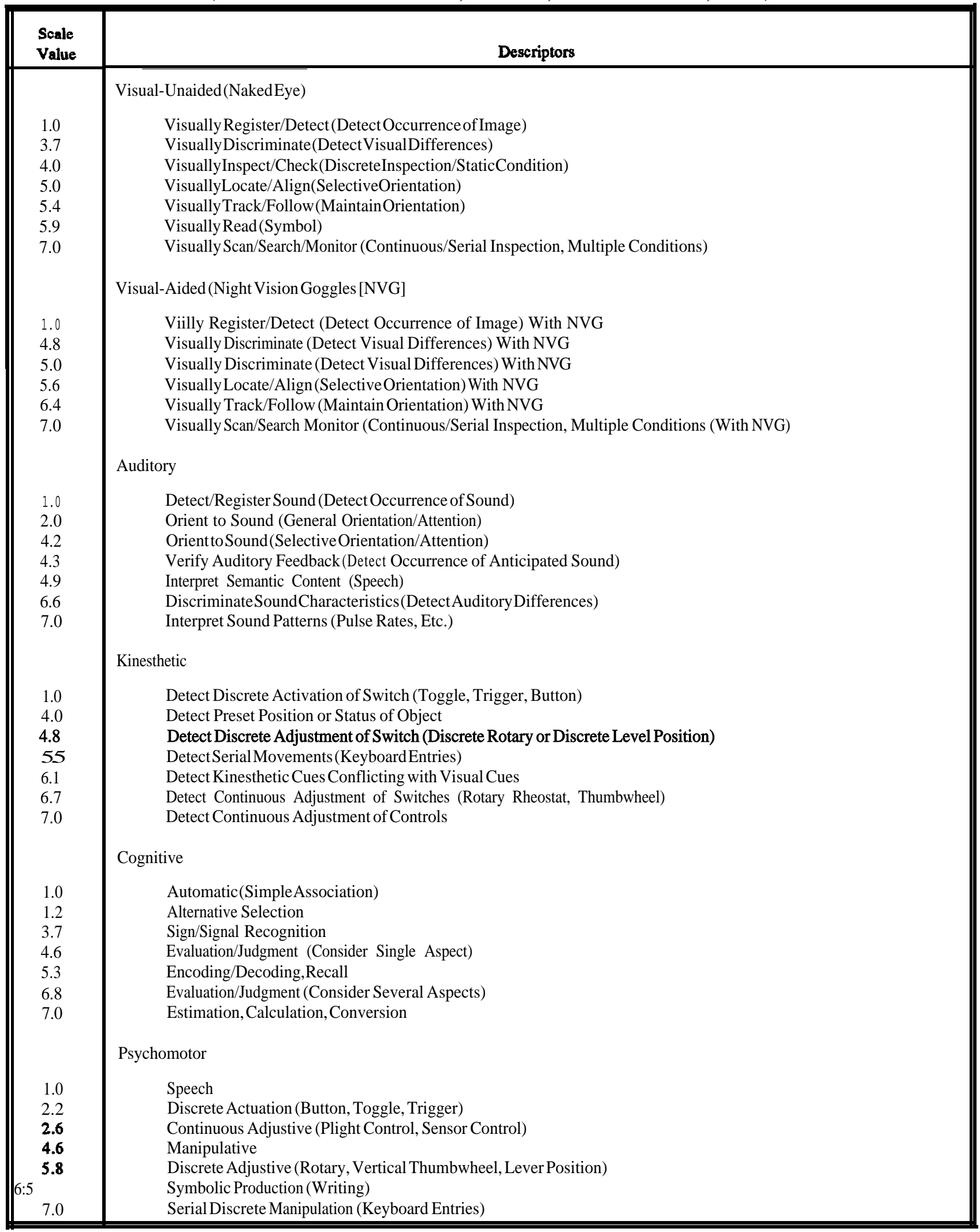




\section{Criticality Ratings and Rankings}

The task descriptions and criticality ratings reflect the use of various methods used in driver task analyses. For example, Spolander (1980) used magnitude estimation to develop subject matter expert (SME) estimates of frequency, safety-criticality, effectiveness (for transportation), and difficulty-tomaster. As briefly described in the English translation of the abstract, the SMEs rated each task on each dimension using a scale from 1 to $100(1=$ least to $100=$ most on that dimension $)$. These ratings were collated, scaled, and turned into integer standard scores.

Moe, et al. (1973) used card sorting procedures and had their SMEs rank driving tasks in terms of criticality. Specifically, thirty-seven (37) truck Driver SMEs and twenty-four (24) bus driver SMEs rated tasks on safety criticality. Each SME was sent three envelopes with 25 randomly selected task descriptions in each envelope. The SME first sorted the 25 task descriptions into three piles of safety criticality, i.e.,

High criticality - Tasks the driver must do

Moderate criticality - Tasks the driver ought to do

Low criticality - Tasks the driver may do

Then the SME rank ordered the 25 tasks from most safety-critical to least safety-critical. Finally, the SME drew a line to mark the High-criticality tasks from the Moderate-criticality task and the Moderate-criticality tasks from the Low-criticality tasks. This procedure was used to identify high criticality driver tasks. Results indicated there was considerable disagreement among SMEs on the rank position of the tasks. Perhaps this was because any given task was ranked relative to the set of randomly selected items with which it was included; differing 'context' would have influenced the rankings.

The ranking and rating methods hold promise for use in the current effort. The assessment of criticality (or other task dimensions to be determined) can probably not be efficiently collected without some recourse to these methods. Inter-judge concordance or reliability is probably important to consider and the effects of various driving conditions may significantly affect the ratings.

\section{Other Techniques}

A variety of other techniques have been used in cognitive science, artificial intelligence, and human factors to uncover the cognitive aspects of tasks and performers (see Appendix A). One class in particular attempts to develop conceptual maps or cognitive graphs of a problem. These graphs depict the relations among concepts that a person uses in a task or the organization of knowledge in memory. The appropriateness of this level of detail for the purposes of workload protocol development are unclear at this time. If the need for such detail is justified, such methods may be used in later phases of this project. 


\section{$\underline{\text { References }}$}

Aldrich, T., Szabo, S., \& Bierbaum, C. (1989). The development of models to predict operator workload during system design. In G. McMillan et al. (Eds.) Applications of human performance models to svstem design (pp. 65 - 80). New York: Plenum Press.

Hannigan, S., \& Parkes, A. (1988). Critical incident driver task analysis. In A. G. Gale et al. (Eds.), Vision in vehicles II (pp. 105 - 114). Amsterdam: Elsevier.

Hart, S. G., \& Wickens, C. D. (1990). Workload assessment and prediction. In H.R. Booher (Ed.), MANPRINT: An approach to svstems integration (pp. 257 - 296). New York: Van Nostrand Reinhold.

Meister, D. (1985). Behavioral analvsis and measurement methods. New York: Wiley.

Rabideau, G., \& Young, P. (1974). Validation of a task analvsis methodology applied to long - haul truck driver behaviour. National Research Council of Canada. (Microfiche No. ED 083 295).

Spolander, K. (1980). Professional driver training for the 1980's. Truck and bus driver task analysis. Linkoping, Sweden: National Swedish Road \& Traffic Research Institute. (In Swedish, Portions Translated by Battelle). 


\subsection{TASK 3 TASK ANALYSIS DATA COLLECTION PLAN}

$\underline{\text { Introduction }}$

A data collection plan for Task 3 is presented in this section. This activity is intended to fill in data gaps which will support the development of the heavy vehicle workload assessment protocol. It departs somewhat from the emphasis included in the original Battelle proposal, i.e., almost exclusive on-the-road data collection. Our proposed changes have come about because of a better understanding of the project requirements and real world limitations associated with on-the-road data collection using commercial drivers on their assigned routes.

\section{$\underline{\text { Objectives }}$}

The objectives of this data collection effort are as follows:

- $\quad$ Evaluate the demand placed on the driver by various driving conditions;

- $\quad$ Determine the safety criticality, relative frequency, and difficulty of selected standard driving tasks and driver behaviors;

- $\quad$ Collect on-the-road data on the visual, manual, and cognitive loads imposed by various tasks and in-cab behaviors found in trucks today; and

- $\quad$ Begin task analysis of selected high-technology in-cab devices to determine the nature of their interactions with drivers and loads which may be placed on drivers.

\section{Scope}

Battelle will restrict the task analysis effort to only those tasks and driver behaviors in which the vehicle is in motion. While important to highway safety in the broadest sense, tasks such as pre-trip planning, before-trip inspections, after-trip inspections, on-the-road repairs, and the like are considered outside the scope of this project.

To the extent possible, we will probe drivers for ancillary driver behaviors which may have safety and workload implications. These include such behaviors as fumbling for snacks, pouring coffee, writing notes into a log book, and so on.

A task analysis will be started on selected examples of high-technology in-cab devices. It is likely that this aspect of the project will continue in greater depth through later phases. This initial task analysis will provide in indication of the nature of device interactions and loads imposed by various kinds of devices to which this protocol will be directed.

Finally, from a workload perspective, tasks or driving situations which involve driver underload will not be actively pursued in this effort. 


\section{$\underline{\text { Annroaches }}$}

In order to meet the objectives listed above, a combination of data collection methods will be used. These include:

- $\quad$ scaling procedures applied to driver assessments of the demand imposed by various driving conditions and relative frequency with this such driving conditions are encountered;

- discussion to elicit driver expert judgements of task safety criticality, frequency, and difficulty;

- $\quad$ on-the-road data collection to gather data on the perceptual, motor, and cognitive loads imposed by various driving tasks and in-cab behaviors found in trucks today; and

a preliminary task analysis of selected high technology in-cab devices.

These methods and a proposed schedule for task analysis data collection are presented below.

Step 1. Evaluate the demand placed on the driver by various driving conditions. The driving condition outside the cab is thought to be the prime determinant of driving demand. Driving conditions can be characterized along a number of dimensions. While there are a large number of different driving conditions which might be encountered, only a selection can be examined in the workload assessment protocol development. This step will involve characterizing driver assessments of the relative demand imposed by various driving conditions by means of conjoint scaling. Drivers will also be asked about the relative frequency with which various driving conditions are encountered. This data will support the selection of driving conditions to be incorporated in subsequent phases of this project.

Step 2. Determine the safety criticality, relative frequency, and difficulty of selected standard driving tasks and driver behaviors. Task criticality, frequency, and difficulty are likely to be contingent on the prevailing driving conditions. Therefore, the results of Step 1 will be used to provide "context" in discussions with drivers about selected driving tasks and driver behaviors in terms of safety criticality, relative frequency, and difficulty. It is likely that drivers will first identify those tasks which are imply high workload and their frequency of occurrence. Additional discussion will address safety criticality and difficulty issues. Data collected in this step will allow us to prioritize tasks which we wish to include as standard tasks in future phases of this project.

Step 3. Collect on-the-road data on the visual, motor, and cognitive loads imposed by various tasks and in-cab behaviors found in trucks today. It is useful for protocol development to have some data on the workload associated with various driving tasks and in-cab behaviors found in trucks today. This type of information can be used to determine the range of visual demands imposed by tasks conducted in trucks without high technology devices, for example, and how such demand changes by driving conditions. This type of data might then be used to direct protocol development or for comparative purposes in analyzing selected in-cab devices. To address the need to obtain preliminary information about driver workload, ride-along observation of truck drivers will be 
conducted. An on-board video recording system will be mounted into the cab of the truck to record the data for this effort.

The ride-along observer may ask the driver to visually scan selected gauges on the instrument panel or manipulate instrument panel controls when the driving task permits. The driver will be instructed that the driver is the final judge on whether or when to respond. Under no circumstances will the driver be asked to perform any action which is not a part of a normal drive. The ride-along observer may also discuss with the driver various issues in heavy vehicle driving (e.g., difficulty or safety criticality of certain tasks, their opinion of how driving conditions affect driving safety, etc.). Battelle will make its best efforts to schedule ride along observations under a variety of driving conditions.

Step 4. Begin task analysis of selected high-technology in-cab devices to determine the nature of their interactions with drivers and loads which may be placed on drivers. In addition to characterizing the driving tasks, it has become apparent that preliminary analysis of selected in-cab devices is also of great use. This analysis, though not originally included in this task, will therefore be started. Data on various classes of devices will be reviewed by the human factors staff, an attempt will be made to obtain one or more devices for static evaluation, and local trucking lines which use such devices can be contacted to begin dialogue on their experiences with them.

\section{Materials and Equipment}

Minimal equipment is needed for Steps 1,2, and 4; Step 4 will benefit by obtaining one or more devices for in-house static evaluations, however. Step 3 requires a video recording system for its completion. The recording system will consist of two video cameras, a video cassette recorder, TV monitor, time generator and an observer's video log. One video camera will be directed toward the road scene and the second camera will be directed to the driver's face to capture gaze location and duration. The observer's log will consist of a panel with thumbwheel switches, each of which provides a digital (O-9) input which will be superimposed on the video tape during recording. This log will allow the observer to enter coded information unobtrusively during taping. The time generator will provide a digital clock to the video scene to facilitate subsequent data analysis (e.g., glance time, glance frequency, inter-glance interval). A video splitter/inserter will be used to combine the views of both cameras into a single input to the VCR.

\section{$\underline{\text { Subject Sample }}$}

Battelle anticipates a sample of 20 to 40 drivers for participation in Steps 1, and 2. Battelle would make best efforts to secure a sample which ranged over the variables of age, sex, and experience. Subjects will be volunteers solicited from area freight, storage, and commercial firms while awaiting warehousemen to load their trailers. For Step 3 over-the-road data collection, Battelle anticipates a sample of 8 to 10 commercial drivers driving on their assigned routes. To the extent possible, a variety of drivers of varying age, experience, and sex will be observed during this portion of the work. Step 4 is not expected to make use of subjects, per se. 
Data Reduction and Analvsis

Conjoint analysis will be used to scale the relative demand of various driving conditions and the contribution different factors make on demand. Driver evaluations of task safety criticality, frequency, and difficulty will be represented in the form of summary statistics. Video tapes will be reviewed to extract visual allocation information and summary statistics will be prepared for these as well. Initial task analysis of selected in-cab devices will be carried out by human factors staff and characterized in tabular form, as appropriate. Table 7.1 provides some indication of the factors which will be evaluated in the in-cab device analysis.

\section{Anticipated Report Format for Task Analvsis Data}

The results of this task analysis effort will be reported in narrative, tabular, graphic, and statistical formats, as appropriate.

\section{Task Analvsis Data Collection Schedule}

A tentative Schedule is provided here. Note that the Draft Task 3 Interim Report delivery date is rescheduled for May 29, 1992.

\section{MONTH \& YEAR}

$\begin{array}{lll}\text { Feb92 } & \text { Mar92 } & \text { Apr92 }\end{array}$

Step 1

Step 2

Step 3

$\mathbf{X}^{*}$

Step 4

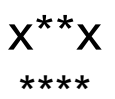

$X^{\star *} X$

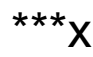

$X^{* *} X$

Draft Report 
TABLE 7.1. DATA GATHERING PROTOCOL FOR NHTSA HV TASK ANALYSIS: IN-CAR DEVICE INTERACTION

\begin{tabular}{|c|c|}
\hline In-cab Device Dimension & Possible Values \\
\hline When used: & $\begin{array}{l}\text { Pre-trip only; when stopped only; backing, in forward motion, } \\
\text { etc. }\end{array}$ \\
\hline $\begin{array}{l}\text { Relevant forward motion } \\
\text { driving tasks: }\end{array}$ & See Task 2 Interim Report for standard driving task. \\
\hline $\begin{array}{l}\text { Driver-initiated or device- } \\
\text { initiated interaction: }\end{array}$ & $\begin{array}{l}\text { E.g., driver elects to glance at speedometer vs. communications } \\
\text { or warning device which "hails" driver. }\end{array}$ \\
\hline Loads placed on driver: & Visual, manual, auditory, cognitive, pedal. \\
\hline $\begin{array}{l}\text { Type of cognitive tasks } \\
\text { reauired: }\end{array}$ & E.g., check reading, text display, typing input, etc. \\
\hline Time required: & In seconds. \\
\hline Error modes: & What can go wrong and likely driver reactions. \\
\hline Task steps: & Indicated, perhaps, by mode of operation. \\
\hline Perseverance effects: & $\begin{array}{l}\text { Device allows driver to readily break the task into segments and } \\
\text { come back to it vs. design which prompts driver to persevere } \\
\text { until task is complete (e.g., because needed data is rolling off the } \\
\text { screen, because system timeouts reset a control, etc.) }\end{array}$ \\
\hline Importance of interaction: & $\begin{array}{l}\text { Perceived criticality (e.g., must do, optional); an indication of the } \\
\text { urgency with which the driver will want to respond to the } \\
\text { devices. }\end{array}$ \\
\hline Positioning: & Where device is mounted: on dash, overhead, on seat, other. \\
\hline $\begin{array}{l}\text { Likelihood of use with } \\
\text { other systems: }\end{array}$ & $\begin{array}{l}\text { What other systems might be used with the device in question. } \\
\text { E.g., a multifunctional/integrated digital communications system } \\
\text { may not be used with a trip recorder (because it already has that } \\
\text { function built into it) but could be used with, say, a cellular } \\
\text { phone. }\end{array}$ \\
\hline
\end{tabular}


APPENDIX A

COGNITIVE TASK ANALYSIS METHODS 


\title{
COGNITIVE TASK ANALYSIS METHODS
}

\author{
Carol A. Tolbert, Ph.D. - Battelle HARC
}

Introduction

Cognitive Task Analysis (CTA) is a host of techniques used to assist in defining the cognitive requirements of tasks. Its purposes are similar to those for traditional, behavioral task analyses: to identify task characteristics and demands for use in developing task hardware, software, training programs, and expert models. While traditional task analyses have used observable behavioral indices, CTA cannot afford this luxury. Instead, CTA must rely on other techniques for identifying and representing the cognitive strategies and structures required for task performance. CTA is actually a complex methodology that consists of:

a) data collection methods, also called 'knowledge elicitation' methods,

b) data analysis methods, and

c) data representation methods.

Each CTA technique that is discussed in the literature typically addresses one or more of the above elements. This can result in confusing terminology regarding what is or is not a CTA method. In this section, we will present an overview of CTA methods that have been used. We will also indicate whether each one is a data collection, analysis, or representation method.

Although "Cognitive Task Analysis" is a relatively new term (coined within the last few years), analyses of cognitive tasks have been performed for decades. The work of Newell and Simon (1972) was a stark indication of CTA. They studied human cognition by having subjects think aloud as they solved problems. The think-aloud verbal protocols were audiotaped and then transcribed for analysis. The analyses revealed, in great detail, how each subject solved each problem. This information was useful for understanding the cognitive limitations of humans and, therefore, the boundaries of expected task (problem) performance. This type of information, in turn, is clearly integral to any task analysis. For example, we know that people have an upper limit on the quantity of incoming information they can cognitively process within a given period of time; thus, a user-interface should not display information to the user at a level that exceeds this limit.

A great deal of research has suggested that people have a variety of cognitive strengths and limitations. For example, they are prone to commit a variety of biases that distort their decisionmaking strategies (e.g., Kahneman, Slovic, \& Tversky, 1982). Memory limitations can also hinder task performance (e.g., Anderson, 1980). Conversely, studies have also shown that students who are provided with methods for organizing concepts and memorizing information perform better than those who are not (Thomas \& Robinson, 1972). Therefore, it is possible to compensate for some limitations by improving other cognitive capabilities. Similarly, research has suggested that even some of the identified limitations can be mitigated through certain instructional strategies (Tolbert, 1989).

Despite these findings, cognitive requirements were not considered for integration into the Instructional Systems Development QSD) paradigm until fairly recently. As a result, traditional taskanalytic methodologies in ISD had already been developed, and did not explicitly address the cognitive limitations of users or the cognitive requirements of the task (Redding, 1990). Methodologies for analyzing the cognitive requirements are relatively young and, consequently, are 
still being developed. The youthfulness of the field is evident everywhere: for example, terms are not standardized, taxonomies of cognitive structures have yet to be established, and implementations and definitions of methodologies vary widely. This suggests that the knowledge base of the field is not sufficiently developed to endorse a unified set of standardized, well-accepted, and reliable methods of CTA. Rather, researchers and practitioners in the fields of ISD, cognitive psychology/science, and human factors are all working to advance CTA methodology, which requires the skills and knowledge in these areas, as well as those in statistics and research methodology (Redding, 1990).

Several methods of CTA have been developed to accommodate data collection, analysis, or representation. In this section, we will limit our discussion to the most widely used methods: verbal protocol analysis, the Critical Decision Method, Concept Mapping, Conceptual Graphs, and psychological scaling methods. For the most part, these methods are not mutually exclusive; in fact, some combinations generate highly effective and complementary approaches to CTA. Each method has strengths and weaknesses that should be considered when deciding on a method.

\section{$\underline{\text { Cognitive Task Analvsis Methods }}$}

\section{$\underline{\text { Verbal Protocol Analvsis }}$}

Verbal protocol analysis (VPA) is a methodology for collecting and analyzing verbal data. Ericsson and Simon (1984) provided an in-depth theoretical framework for conducting VPA; many researchers have used it to conduct VPA studies of their own. VPA typically involves the following activities:

- A person is given a task to perform.

- $\quad$ The person performs the task and thinks aloud while performing the task ("concurrent" VPA).

- $\quad$ The person's verbalizations are audiotaped.

- The person's audiotape is transcribed.

- The person's transcription is analyzed.

- $\quad$ Conclusions are drawn about the cognitive processes the person applied while performing the task.

A variation of this general approach is to require the person to verbalize how he performed a task some time after he performed it ("retrospective" VPA). Another variation is to videotape the session; the extra visual information can be valuable in the data analysis. An important consideration that affects the entire methodology is the task that is chosen. Ericsson and Simon (1984) suggested that task characteristics, such as task difficulty and automaticity, be maintained in a preferred range. If the task is too difficult, thought processes and verbalization can be hindered. If the task is too easy, the person might perform it automatically. In this case the person would be unable to verbalize his thinking because he would be executing the task without conscious awareness.

A criterion for verbalizing one's thoughts, according to Ericsson and Simon (1984), is that they be available for retrieval from short-term memory. Therefore, cognitions that a) cannot be retrieved from long-term memory, b) are not attended to by the person, or c) are otherwise at a non-conscious level, cannot exist in short-term memory and are thus not verbalizable. 
The analysis of verbal protocols is more challenging than their collection and no standardized techniques exist. Close-ended tasks with well-defined problem spaces are the easiest to analyze, while open-ended tasks with undefined problem spaces are the most difficult to analyze. The theoretical framework of Ericsson and Simon (1984) did not address undefined tasks; however, open-ended tasks have been analyzed (e.g., Tolbert, 1985). Such novel applications are central to understanding how people in normal jobs solve daily tasks because most of them are open-ended.

The analysis of verbal protocols (close-ended tasks) usually includes the following activities:

- $\quad$ defining the vocabulary of terms used in the verbalizations

- $\quad$ testing and refining the vocabulary

- $\quad$ segmenting the audiotape transcriptions into semantic chunks

- $\quad$ encoding the segments by applying the vocabulary

The technique(s) chosen for summarizing and presenting the results is at the researcher's discretion (e.g., tables, graphs, verbal summaries), depending upon the goals of the project and what information the researcher is trying to convey. In any case, the selected format should be able to summarize the thought processes used in performing the given task.

The information obtained from VPA can be used in a variety of ways to assist researchers, cognitive scientists, ergonomists, and systems engineers. Researchers have used the method to understand how people solve problems and make decisions (e.g., Bhaskar \& Simon, 1977; De Groot, 1965; Duncker, 1926; Newell \& Simon, 1972; Watson, 1920). Cognitive scientists have used VPA to acquire expert domain knowledge for building models of expert performance (e.g., Roth, Bennett, \& Woods, 1987; Roth \& Woods, 1988). Ergonomists have only recently begun to explore VPA as a method for helping define task requirements and evaluating existing systems (e.g., Bowers \& Snyder, 1990; Denning, Hoiem, Simpson, \& Sullivan, 1990). Systems engineers, similarly, could work with ergonomists to use VPA in the requirements definition and design phases of system development. In a recent paper, Tolbert and Bittner (1991) explored the potential uses of VPA throughout the systems development cycle: during conceptual design, after system start-up (during operation), and after specific incidents (during operation) to help identify problem sources.

VPA provides comprehensive and detailed analyses of cognitive performance on tasks. This information is crucial for understanding the cognitive components of task performance. It uncovers both the knowledge and the strategies used to perform the task. Unfortunately, the cost of the method's comprehensiveness is that it is resource-intensive, requiring extra time, effort, and special skills. In the absence of one or more of these resources, VPA results can produce misleading or questionable conclusions. Because standardized methods of VPA have not been adopted, the method is prone to criticism.

\section{Critical Decision Method}

Developed by Klein, Calderwood, and Clinton-Cirocco (1986), the Critical Decision Method (CDM) is a method of eliciting knowledge. It was derived from Flanagan's Critical Incident Technique (1954). CDM elicits the deeper knowledge that can be difficult to retrieve through other methods. For this reason, CDM is often used to elicit the knowledge of experts. It is thus a data collection method, using the elicited cognitions of experts to analyze cognitive task requirements. 
The following procedures are used in CDM (Thordsen, 1991):

1. The person is asked to recall a specific incident that occurred in the past in which the person's expertise was challenged or affected the outcome of the incident.

2. The person then verbalizes his recollection of the incident including, for example, a description of the incident, his role, actions, thoughts, and observations during the incident.

3. A chronology of the incident is developed based on the person's recollections.

4. The person is questioned to fill in details or gaps suggested by the chronology. Examples include questions about decisions that were made and their bases, as well as where specific information was obtained.

CDM can be viewed as an abbreviated form of retrospective VPA, similar to that described in the foregoing VPA subsection.

By focusing on unusual events, experts are able to recall the event and its surrounding circumstances. Unusual events require the expert's unique skills and knowledge to be utilized. CDM capitalizes on this requirement in order to reveal the expert's deep knowledge and refined skills used to help resolve specific incidents. This feature is particularly useful because research has demonstrated that experts chunk their knowledge (in ways that novices do not) and automate their knowledge retrieval strategies (e.g., Chase \& Ericsson, 1982; De Groot, 1965). As a result, their cognitive skills and knowledge are not available for conscious retrieval by the expert. According to Klein and Crandall (1990), $\mathrm{CDM}$ is an effective method for indirectly accessing the expert's expertise.

An advantage of CDM is therefore its ability to elicit experts' deep knowledge and skills (Thordsen, Wolf, \& Crandall, 1990). It is also practical to apply because it can be performed anytime after the incident, in almost any locale. However, these practical assets are also liabilities: the longer the time between the incident and the time of recollection, the more the expert's memory for the incident will become degraded and distorted. Still, the flexibility offered by CDM is a unique benefit.

\section{Concert Mapping}

Concept Mapping is a method of data collection and data representation. Originally proposed by Gowin and Novak (1984), it has recently been used to elicit domain expertise (Wolf, Klein, Thordsen, \& Klinger, 1991) and to analyze user needs and develop workstation designs (e.g., McFarren, 1987).

Concept Mapping uses interviewing as its tool for eliciting knowledge from an expert. Typically, an expert and a knowledge engineer work together to develop a "Concept Map". Before the Concept Mapping session, the knowledge engineer identifies the task domain that will be queried and the expert who will participate. The session begins with the knowledge engineer indicating the task domain to the expert. The expert is asked to identify the key concepts involved in performing the task, as he understands them. For example, key concepts involved in 'going grocery shopping' might 
include making a list, driving to and from the store, finding the items on the list, and paying for them. Another person's key concepts for this task might be different, excluding making a list, and taking the bus to and from the store.

As the expert provides his key concepts, the knowledge engineer begins to draw a diagram that illustrates the concepts and the relationships among the concepts. Concepts are drawn as nodes, and relationships are drawn as directional arrows connecting the appropriate nodes. The knowledge engineer continues to query the expert to reveal the finer concepts and to ensure that all relationships

and concepts are represented. This iterative process between the knowledge engineer, the expert's responses, and the knowledge engineer's adjustments to the Concept Map continue until the level of detail provided matches the needs of the knowledge engineer.

Concept Mapping results in a visual representation of an expert's perspective on performing a particular task. Representations such as these have been used for many years by cognitive theorists to represent knowledge [e.g., Anderson's "propositional networks" (1980)]. They are a useful and concise method of portraying the abstractions inherent in analyzing human cognition. However, they can become unduly large and complex, thus losing the advantage of conciseness. This must be avoided by carefully selecting and defining the task to be explored.

As presently used, Concept Maps do not capture the multidimensional and dynamic nature of many experts' thinking. Furthermore, they may not reveal the hidden knowledge and skills of the expert (i.e., automated skills). To address this concern, Thordsen (1991) suggests that Concept Mapping be conducted along with the Critical Decision Method. Thordsen contends that Concept Mapping can provide an overview structure of the expert's thinking, while CDM can elicit the deeper knowledge and skills needed to fill in the Concept Map.

\section{Conceptual Graphs}

Conceptual graphs, like Concept Maps, are visual representations of a person's knowledge. Conceptual graphs also consist of concepts, represented by nodes, and the relationships among concepts, represented by directional arrows. They are a generic type of knowledge representation, produced through any of several data collection methods, including interviewing. Moore and Gordon (1988) adapted a "question-probe" method, originally developed by Graesser (Graesser \& Clark, 1985; Graesser \& Goodman, 1985), to be used as a data collection method to generate conceptual graphs. In instructional applications, Moore and Gordon combined the question-probe and conceptual graph methods to develop instructions, diagnose student comprehension (Gordon, Gill, \& Moore, 1988), and assess the effects of different instructional techniques (Gill, Gordon, Moore, \& Barbera, 1988).

For use in prose comprehension, the combined method begins by having subjects read a passage of text. Then they are queried about the passage: For each event in the passage, subjects must indicate how and why the event occurred, as well as what happened next. The subject's responses are transformed into a conceptual graph that depicts his understanding of the passage.

In Moore and Gordon's (1988) application of the combined method, they proposed two specific question-probing techniques for eliciting information from a subject. The first technique is subjectdriven. A task domain is identified and a conceptual graph is developed by both the knowledge 
engineer and the subject. The subject is given an initial, higher-level concept and asked questions to elicit information - that is, additional concepts -- about the initial concept. Next, the additional concepts provided by the subject are used as question probes to generate the next set of concepts. In this way, the subject drives the definition of the conceptual graph.

The second technique uses a set of predetermined questions to guide the construction of the subject's conceptual graph. In this application, all nodes of a standard conceptual graph have been defined prior to the session. The subject is first probed on the first concept in the standard graph, then on the second concept in the graph, and so on. This process results in a second conceptual graph, that of the subject, which can be compared to the standard.

In addition to various instructional applications, these techniques can be used to elicit expert knowledge for expert systems or user knowledge for specific system development or modification activities. Like Concept Mapping, the techniques produce visual representations of a person's cognitive framework for a given task domain. They are sufficiently generic to be adaptable to a wide array of ergonomic applications that could benefit from cognitive task analyses. The disadvantages of the techniques are similar to those of Concept Mapping, that is, the multidimensional and dynamic nature of cognitive activities is underscored, and cognitive skills or knowledge of which the person is unaware cannot be directly revealed.

\section{Psychological Scaling Methods}

Psychological scaling methods are indirect methods for collecting cognitive data. Many of them require the subject to make similarity judgments, rating judgments, or sorting judgments on predefined sets of task-relevant stimuli (concepts). An underlying assumption made by researchers using these methods is that a subject's internal organization of concepts regarding a task domain can be inferred from the subject's externalized judgments, which are based on the provided sets of stimuli.

After subjects provide their judgments, the externalized data are typically converted into pair-wise comparisons among the concepts. The pair-wise comparisons are then statistically analyzed using multidimensional scaling, cluster analysis, or ordered trees. These analyses organize the judgments according to their levels of similarity and hierarchical ordering. The researcher uses this output to make inferences about the subject's cognitive structures (e.g., mental models, schemata, knowledge organization).

Psychological scaling methods have been widely used to conduct cognitive task analyses. The methods are relatively easy to execute because the subject or expert does not have to devote copious amounts of time and the analyses are run on statistical packages. In addition, they purportedly measure cognitive organization that is deep and difficult to verbalize. However, many researchers have come to realize that the methods are highly questionable. This is primarily because of the large number of subjective judgments that are made by the researcher, beginning with the subject's actual cognitions and ending with the researcher's final depiction of those cognitions (Redding, 1989). Consequently, these methods are best utilized in conjunction with other CTA methods. 


\section{$\underline{\text { Summarv of Methods }}$}

Verbal protocol analysis is the most comprehensive and direct method for eliciting and analyzing a person's cognitive task performance. It yields an almost uninterrupted, sequential, stream of thought that can reveal a person's knowledge, skills, and strategies used in a given task domain. It also is the most resource-intensive, requiring careful and time-consuming analysis.

The Critical Decision Method is useful for eliciting an expert's knowledge and skills, especially those that are tacit and unavailable for direct verbalization. It is the only method that uses an actual series of events to retrieve the expert's behavior. If the expert's memory is good, and can be corroborated by other sources, then the method can provide a direct and unique elicitation of the expert's deep cognitions. If the expert's memory is bad or distorted, then the method fails. However, if the expert's recollection is inaccurate, then the knowledge engineer/researcher might unknowingly make erroneous conclusions about the expert's cognitive structures.

Concept Mapping and conceptual graphs both rely on interviewing techniques to elicit information from the subject or expert. The information is used to construct visual representations of the subject's cognitive approach to performing the task. These methods are effective tools for eliciting and representing cognitive structures. However, the sequence that the subject would follow during actual performance of the task is omitted, as is the multidimensional and dynamic characteristics of subjects' cognitiveperformance. The methods are effective as long as the representations are viewed as overall structures that must be accompanied by other methods to provide the omitted cognitive characteristics.

Psychological scaling methods are indirect data collection methods used to uncover subjects' cognitive organizations about a set of concepts. Subjects' judgments are elicited, transformed, and analyzed using multivariate statistical procedures. The output is used to infer subjects' conceptual organizations. These methods are well-suited to identify clusters of relatively simple concepts. Inferences beyond subjects' judgments, however, are questionable and could lead to inaccurate representations of subjects' cognitions. Moreover, the methods do not elicit much information from the subject, especially compared to the richness that is found in subjects' cognitions.

\section{$\underline{\text { Conclusions }}$}

The decision to apply one or more of the methods depends upon many factors, such as resource constraints and project goals. Ideally, more than one method would always be used, as an attempt to convergemethods. CDM, for instance, could complement any of the methods, as could psychological scaling methods. Interviewing techniques can also complement almost any method. Or, as suggested earlier, interviewing can be the primary method of knowledge elicitation. In order to apply them successfully, all the methods require special expertise (Redding, 1989, 1990) because they are complex and otherwise error-prone.

The methods discussed in this section have been used by various researchers to analyze the cognitive requirements of tasks. Each method can contribute unique information about a person's cognitive structures. However, none of these methods, nor any other CTA method, has been standardized. Nor have any formal, widely accepted procedures for executing CTA been developed. Nonetheless, the development and application of these methods demonstrate great progress in incorporating cognitive requirements into routine task analyses. That cognitive requirements were ever omitted is 
quite perplexing, considering that almost all tasks performed by humans require a certain amount of cognitive resources. Even so-called low-level jobs, such as assembly line work, require a modicum of decision making effort. It is therefore crucial that cognitive requirements be taken into account when task requirements are being analyzed. 


\section{$\underline{\text { References }}$}

Anderson, J. R. (1980). Cognitive psychology and its implications. San Francisco: W. H. Freeman and Company.

Bhaskar, R., \& Simon, H. A. (1977). Problem solving in semantically rich domains: An example from engineering thermodynamics. Cognitive Science, 1, 193-215.

Bowers, V. A., \& Snyder, H. L. (1990). Concurrent versus retrospective verbal protocol for comparing window usability. In Proceedings of the Human Factors Societv 34th Annual Meeting, pp. 1270-1274. Santa Monica, CA: Human Factors Society.

Chase, W. G., \& Ericsson, K. A. (1982). Skill and working memory. In G. H. Bower (Ed.), The psychology of learning and motivation (Vol. 161. New York: Academic Press.

De Groot, A. D. (1965). Thought and choice in chess. The Hague: Mouton.

Denning, S., Hoiem, D., Simpson, M., \& Sullivan, K. (1990). The value of thinking-aloud protocols in industry: A case study at Microsoft Corporation. In Proceedings of the Human Factors Societv 34th Annual Meeting, pp. 1285-1289. Santa Monica, CA: Human Factors Society.

Duncker, K. A. (1926). A qualitative (experimental and theoretical) study of productive thinking (solving of comprehensible problems). Pedagogical Seminary, 33, 642-708.

Ericsson, K. A., \& Simon, H. A. (1984). Protocol analvsis. Cambridge: MA: The MIT Press.

Flanagan, J. C. (1954). The critical incident technique. Psychological Bulletin, 51, 327-358.

Gill, R., Gordon, S., Moore, J., \& Barbera, C. (1988). The role of conceptual structures in problem solving. In Proceedings. Annual Meeting of the American Societv of Engineering Education.

Gordon, S., Gill, R., \& Moore, J. (1988). The use of auestion probes for manning conceptual structures. Manuscript submitted for publication.

Gowin, R., \& Novak, J. D. (1984). Learning how to learn. NY: Cambridge University Press.

Graesser, A., \& Clark, L. (1985). Structures and procedures of implicit knowledge. Norwood, NJ: Ablex.

Graesser, A., \& Goodman, S. (1985). Implicit knowledge, question answering, and the representation of expository text. In B. K. Britton \& J. B. Black (Eds.), Understanding exnositorv text. Hillsdale, NJ: Lawrence Erlbaum Associates.

Kahneman, D., Slovic, P., \& Tversky, A. (Eds.). (1982). Judgment under uncertainty: Heuristics and biases. Cambridge, MA: Cambridge University Press. 
Klein, G. A., Calderwood, R., \& Clinton-Cirocco, A. (1986). Rapid decision making on the fireground. In Proceedings of the Human Factors Societv 30th Annual Meeting, pp. 576-580. Dayton, OH: Human Factors Society.

Klein, G. A., \& Crandall, B. (1990). Applying knowledge engineering to training and technology transfer. In Proceedings of the Human Factors Societv 34th Annual Meeting, pp. 1318-1322. Santa Monica, CA: Human Factors Society.

McFarren, M. R. (1987). Using concept maps to define problems and identify key kernels during the develonment of a decision support svstem. Unpublished Master's Thesis, Air Force Institute of Technology, Dayton, $\mathrm{OH}$.

Moore, J. L., \& Gordon, S. E. (1988). Conceptual graphs as instructional tools. In Proceedings of the Human Factors Societv 32nd Annual Meeting, pp. 1289-1293. Santa Monica, CA: Human Factors Society.

Newell, A., \& Simon, H. A. (1972). Human problem solving. Englewood Cliffs, NJ: PrenticeHall.

Redding, R. E. (1989). Perspectives on cognitive task analysis: The state of the state of the art. In Proceedings of the Human Factors Societv 33rd Annual Meeting, pp. 1348-1352. Santa Monica, CA: Human Factors Society.

Redding, R. E. (1990). Taking cognitive task analysis into the field: Bridging the gap from research to application. In Proceedings of the Human Factors Societv 34th Annual Meeting, pp. 13041308. Santa Monica, CA: Human Factors Society.

Roth, E., Bennett, K., \& Woods, D. D. (1987). Human interaction with an 'intelligent' machine. International Journal of Man-Machine Studies, 27, 479-525.

Roth, E., \& Woods, D. D. (1988). Cognitive task analysis: An approach to knowledge acquisition for intelligent system design. In G. Guida \& C. Tasso (Eds.), Tonics in expertsvstems design. Amsterdam: North-Holland.

Thomas, E. L., \& Robinson, H. A. (1972). Improving reading in everv class: A sourcebook for teachers. Boston: Allyn and Bacon.

Thordsen, M. L. (1991). A comparison of two tools for cognitive task analysis: Concept Mapping and the Critical Decision Method. In Proceedings of the Human Factors Societv 35th Annual Slecting, 2183-285. CA: Human Factors Society.

Thordsen, M. L., Wolf, S. P., \& Crandall, B. (1990). User-defined requirements for the JSTARS self-defense suite. Final report prepared under Contract F33615-88-D-0536. Dayton, OH: Unisys Corporation.

Tolbert, C. A. (1985). Hwothesis testing: in an open-ended problem space. Manuscript in preparation. Tolbert, C. A. (1989). Can a reduced selection task be used to induce 
disconfirmatorv resnonses? Doctoral dissertation, Bowling Green State University, Bowling Green, $\mathrm{OH}$.

Tolbert, C. A., \& Bittner Jr., A. C. (1991). Applications of verbal protocol analysis during the system development cycle. In W. Karwowski \& J. W. Yates (Eds.), Advances in Industrial Ergonomics and Safetv III. London: Taylor \& Francis.

Watson, J. B. (1920). Is thinking merely the action of language mechanisms? British Journal of Psychology, 11, 87-104.

Wolf, S., Klein, G., Thordsen, M., \& Klinger, D. (1991). Factors affecting the decision making of fire direction officers. Final Technical Report prepared under Contract DAAA15-90-C-1054. Yellow Springs, $\mathrm{OH}$ : Klein Associates, Inc. 